OPEN ACCESS

Edited by:

Walter Jäger,

Universität Wien, Austria

Reviewed by:

Ashwini Chand,

Olivia Newton-John Cancer Research

Institute, Australia

Philippa Saunders,

University of Edinburgh,

United Kingdom

*Correspondence:

Andrea Romano

a.romano@maastrichtuniversity.n!

†These authors have contributed equally to this work

Specialty section:

This article was submitted to

Experimental Pharmacology and Drug

Discovery,

a section of the journal

Frontiers in Pharmacology

Received: 28 March 2018

Accepted: 02 August 2018

Published: 19 September 2018

Citation:

Konings G, Brentjens L, Delvoux B,

Linnanen T, Cornel K, Koskimies $P$,

Bongers $M$, Kruitwagen $R$

Xanthoulea S and Romano A (2018)

Intracrine Regulation of Estrogen and

Other Sex Steroid Levels in

Endometrium and Non-gynecological

Tissues; Pathology, Physiology, and

Drug Discovery.

Front. Pharmacol. 9:940.

doi: 10.3389/fphar.2018.00940

\section{Intracrine Regulation of Estrogen and Other Sex Steroid Levels in Endometrium and Non-gynecological Tissues; Pathology, Physiology, and Drug Discovery}

\author{
Gonda Konings $^{1,2 t}$, Linda Brentjens ${ }^{1,2 t}$, Bert Delvoux ${ }^{1,2}$, Tero Linnanen ${ }^{3}$, Karlijn Cornel ${ }^{1,2}$, \\ Pasi Koskimies $^{3}$, Marlies Bongers ${ }^{1,2}$, Roy Kruitwagen ${ }^{1,2}$, Sofia Xanthoulea ${ }^{1,2}$ and \\ Andrea Romano ${ }^{1,2 *}$ \\ ${ }^{1}$ GROW-School for Oncology and Developmental Biology, Maastricht University, Maastricht, Netherlands, ${ }^{2}$ Department of \\ Obstetrics and Gynaecology, Maastricht University Medical Centre, Maastricht, Netherlands, ${ }^{3}$ Forendo Pharma Ltd., Turku, \\ Finland
}

Our understanding of the intracrine (or local) regulation of estrogen and other steroid synthesis and degradation expanded in the last decades, also thanks to recent technological advances in chromatography mass-spectrometry. Estrogen responsive tissues and organs are not passive receivers of the pool of steroids present in the blood but they can actively modify the intra-tissue steroid concentrations. This allows fine-tuning the exposure of responsive tissues and organs to estrogens and other steroids in order to best respond to the physiological needs of each specific organ. Deviations in such intracrine control can lead to unbalanced steroid hormone exposure and disturbances. Through a systematic bibliographic search on the expression of the intracrine enzymes in various tissues, this review gives an up-to-date view of the intracrine estrogen metabolisms, and to a lesser extent that of progestogens and androgens, in the lower female genital tract, including the physiological control of endometrial functions, receptivity, menopausal status and related pathological conditions. An overview of the intracrine regulation in extra gynecological tissues such as the lungs, gastrointestinal tract, brain, colon and bone is given. Current therapeutic approaches aimed at interfering with these metabolisms and future perspectives are discussed.

Keywords: intracrinology, endometrium, estrogens, lungs, gastrointestinal tract, central nervous system, bone

\section{INTRODUCTION}

The term "intracrinology," coined in 1988 by prof Labrie, refers to the ability of peripheral tissues to use blood precursors and generate steroids (Labrie, 1991). Several studies have been published but several controversies still exist and relate to the following technical and biological aspects: (a) some intracrine enzymes in peripheral tissues have low expression (300-50,000-times lower than in endocrine glands Stoffel-Wagner, 2001; Murakami et al., 2006, close to the detection limit of standard methods like western blotting and immunohistochemistry -IHC); (b) the technology 
to robustly quantify steroids (liquid-/gas-chromatography tandem mass-spectrometry -LC-MS or GC-MS), became available during the last 5-10 years only (Rosner et al., 2013); (c) intracrine pathways are highly complex.

This review summarizes our knowledge of intracrinology in peripheral tissues like the endometrium, lungs, gastrointestinal tract (GIT), bone and central nervous system (CNS), with special attention to the metabolism of estrogens. Drug development and potential therapeutic approaches are discussed. In this review, the enzymes involved in steroid deactivation/clearance (Rižner, 2013, 2016; with the exclusion of steroid sulphotransferases) and those involved in the transport of conjugated steroids through the plasma membrane (Rižner et al., 2017) are not described. Studies on serum/tissue steroid levels are reported and discussed only if based on gold standard GC/LCMS.

\section{FROM OVARIAN ESTROGEN SYNTHESIS TO INTRACRINOLOGY}

Local steroid metabolism is possible because those enzymes responsible for steroid synthesis in classical glands (ovaries, adrenals, testes) are expressed in peripheral tissues, where additional and alternative routes for metabolizing steroids are present and make intracrine networks intricate and flexible (Figures 1, 2, Tables 1, 2). In particular, several compounds generated through these pathways, although not being estrogens, can have estrogenic action, because able to bind and activate the estrogen receptors. The biologic activity of the various compounds is given in Table 1, and in Figure 2, by the color codes.

\section{Ovarian Steroidogenesis}

Transformation of cholesterol to $17 \beta$-estradiol (E2) involves first the production of dehydroepiandrosterone (DHEA)

Abbreviations: $[3 \mathrm{H}]$, tritiated hydrogen; [14C], radioactive carbon; $\mathrm{AD}$, Alzheimer disease; ADHD, attention deficit hyperactivity disorder; AI, aromatase inhibitor; AKR, aldo-ketoreductase; AR, androgen-receptor; ART, assisted reproduction technology; $\mathrm{BMD}$, bone mineral density; BMP-2, bone morphogenetic protein 2; COPD, chronic obstructive pulmonary disease; COUPTFII, chicken-ovalbumin-upstream-promoter-transcription-factor II; CNS, central nervous system; CRC, colorectal cancer; CX43, connexin 43; DS, digestive system; Ed, embryonic day; EC, endometrial cancer; EndRet, endoplasmic reticulum; ER, estrogen-receptor; Fdx, ferredoxin; FdR, ferredoxin reductase; GC-MS, gas-chromatography tandem mass-spectrometry; GnRH, gonadotropin releasing hormone; GH, growth hormone; GIT, gastrointestinal tract; GPER, G protein-coupled estrogen-receptor; HPLC, high performance liquidchromatography; HRT, hormone-replacement-therapy; hCG, human chorionic gonadotropin; IGF1, insulin-like growth factor 1; IHC, immunohistochemistry; IL-1, interleukin-1; IL-6, interleukin-6; KO, knock-out; LC-MS, liquidchromatography tandem mass-spectrometry; mTOR, mammalian target of rapamycin; NAPDH, nicotine-adenine-dinucleotide-phosphate; NSCLC, nonsmall cell lung cancer; PAIN, phenomena of pan-assay interfering compounds; PAP, bis-phospho-nucleotide- $3^{\prime}$-phospho-adenosine- $5^{\prime}$-phosphate; PCOC, poly cystic ovarian syndrome; POR, P450 oxidoreductase; RT-qPCR, reversetranscriptase quantitative polymerase-chain-reaction; SF1, steroidogenic factor 1; SHBG, sex hormone binding globulin; SNP, single nucleotide polymorphism; SRD, short-chain dehydrogenase; Th, T-helper; TNF, tumor necrosis factor; VEGF, vascular endothelial growth factor; WB, western blot/blotting; WOI, window of implantation. in theca cells through the action of steroidogenic acute regulatory protein (StAR) that facilitates the transport of cholesterol into mitochondria, followed by CYP11A1 (ratelimiting) and CYP17A1 (Figure 2); the ovarian pathway is indicated by the yellow background; reviewed by (Miller and Auchus, 2011; Andersen and Ezcurra, 2014). CYP11A1 is a type I CYP localized in mitochondria that uses nicotineadenine-dinucleotide-phosphate (NADPH) and ferredoxin $(\mathrm{Fdx}) /$ ferredoxin reductase $(\mathrm{FdR})$ to cleave the cholesterol side chain and produce pregnenolone (P5). Type II CYP17A1, localized in the endoplasmic reticulum (EndRet), has both $17 \alpha$-hydroxylase and 17,20-lyase activities. It uses $\mathrm{NADPH}$ and P450 oxidoreductase (POR) to first hydroxylate P5 to $17 \alpha$-hydroxypregnenolone (17OHP5) (17 $\alpha$-hydroxylase action), followed by 17,20-lyase action to release DHEA. Gonad specific type $23 \beta$-hydroxysteroid dehydrogenase (3ßHSD2) has $3 \beta$-dehydrogenase and $\Delta^{5}$ to $\Delta^{4}$ isomerase activities and converts DHEA to androstenedione (A4). Next, CYP19A1 catalyzes the oxidative demethylation of $\mathrm{C}_{19}$ androgens to $\mathrm{C}_{18}$ estrogens, with A-ring aromatisation; hence A4 is converted to estrone (E1). The final conversion of E1 (with low affinity for the estrogen-receptors -ERs) to E2 (high affinity for ERs and high estrogenic potency) is catalyzed by $17 \beta$ HSD 1 that reduces 17 -keto to $17 \beta$-hydroxyl steroids. In the ovary, the 17-keto group of A4 can be reduced to $17 \beta$-hydroxyl by AKR1C3/17ßHSD5 yielding testosterone $(\mathrm{T})$ that is converted

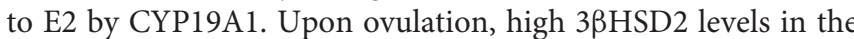
corpus luteum lead to high progesterone $(\mathrm{P})$ generation from P5.

\section{Intracrine Steroidogenesis}

The expression of StAR, CYP11A1 and CYP17A1 is demonstrated in a limited number of peripheral tissues (see later and Tables 6-8). However, pregnenes, pregnanes, androstenes and androstanes generated from these initial steps (but also abundantly available as circulating precursors) can be further metabolized locally thus generating a plethora of compounds with various biological activities (estrogenic, androgenic, progestogenic and neuroactive; Tables 1, 2 and Figure 2). The $\Delta^{5}$ to $\Delta^{4}$ isomerization of androstenes (DHEA, androstenediol -A5- and $17 \alpha \mathrm{A} 5)$ and pregnenes (P5, 17OHP5) is catalyzed by $3 \beta \mathrm{HSD} 1$, which is the peripheral counterpart of ovarian $3 \beta \mathrm{HSD} 2$. Also $3 \beta \mathrm{HSD} 2$, whose expression was initially considered to be restricted to endocrine tissues, is detected peripherally in recent reports (Stoffel-Wagner, 2001; Tsai et al., 2001; Attar et al., 2009; Huhtinen et al., 2014; Osinski et al., 2018). Due to the high concentration of DHEA (both in blood and tissues), its conversion to A4 by $3 \beta \mathrm{HSDs}$ is relevant to the formation of downstream androgens and of estrogens. Additionally, 3ßHSDs convert A5 and the isomer $17 \alpha \mathrm{A} 5$ to $\mathrm{T}$ and epitestosterone (EpiT). Although minor, in the context of women's health, these pathways are relevant. A5, together with $3 \alpha$ and $3 \beta \mathrm{DIOL}$ (generated by AKR1Cs from DHT and AN, see below) activate both ERs and have estrogenic action (especially $3 \beta \mathrm{DIOL}$, a potent ER $\beta$ binder). A5 possesses immune stimulatory activity whereas its $17 \alpha$ isomer $(17 \alpha \mathrm{A} 5)$ has androgenic, antitumor and neuroactivity. 

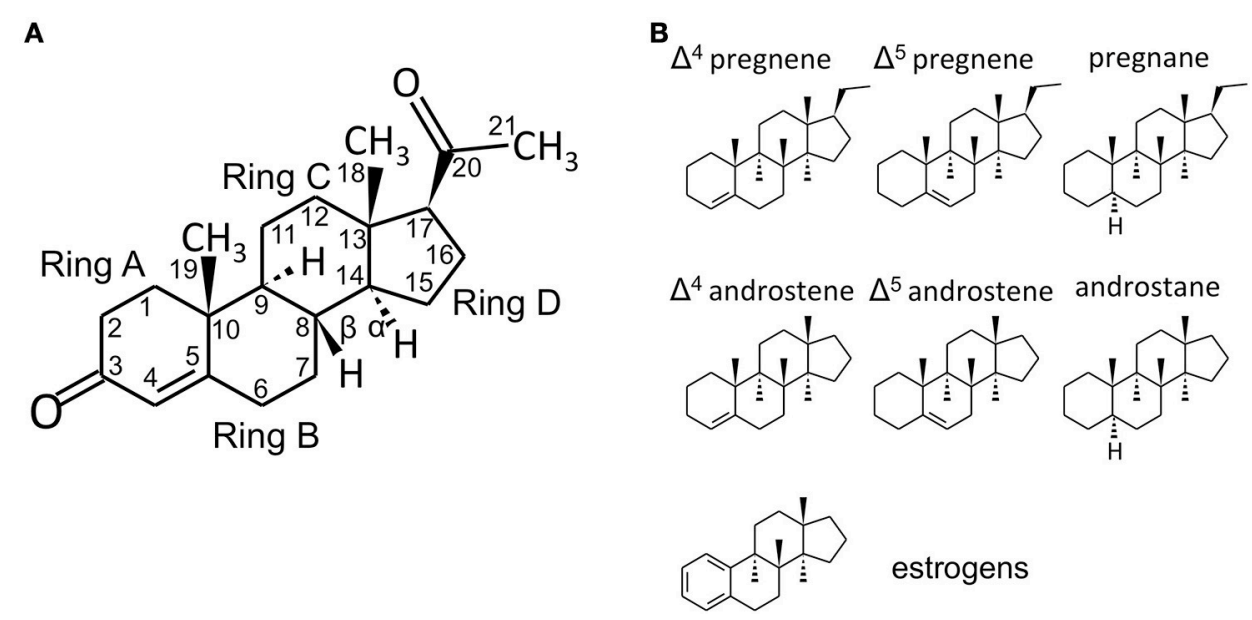

FIGURE 1 | Steroid structure. (A) structure of the C21 steroid progesterone (P, used as an example), with carbon numbering and steroid ring numbering. In the storied graphics in Figures $\mathbf{1 B}$ and $\mathbf{2}$, the $\mathrm{H}$ groups and the relative bonds will be omitted (with the exclusion of the $\mathrm{H}$ in $5 \alpha$-reduced steroids - androstanes and pregnanes). Methyl groups will be indicated by the bonds only without the $\mathrm{CH}_{3}$ group. (B) structures of $\mathrm{C} 21$ pregnene $\left(\Delta^{4}\right.$ and $\Delta^{5}$, i.e., double bond between $\mathrm{C} 4$ and C5 or between C5 and C6, respectively), pregnane (5 $\alpha$-reduced steroid), C19 androstene $\left(\Delta^{4}, \Delta^{5}\right.$ ) and androstane and C18 (A-ring)-aromatic estrogens. Chemical structures were designed with the aid of Sketcher V2.4 (Ihlenfeldt et al., 2009), available online at PubChem (www.ncbi.nIm.nih.gov; pubchem.ncbi.nlm.nih.gov) (Kim et al., 2016).

Additionally, EpiT is a weak AR binder and a strong endogenous inhibitor of SRD5As (Loria and Graf, 2012). The endogenous occurrence of $17 \alpha \mathrm{A} 5$ is demonstrated in humans (Laatikainen et al., 1971) but its route of synthesis is unclear (Shimizu, 1979). A $17 \alpha H S D$ able to convert A4 to EpiT and DHEA to $17 \alpha \mathrm{A} 5$ is characterized in mice (Bellemare et al., 2005) but no human homologous is described yet. Similarly to the ovaries, androgen to estrogen conversion is catalyzed by CYP19A1.

A particularly important reaction is controlled by oxidative and reductive $17 \beta$ HSDs, which interconvert 17 -keto and $17 \beta$ hydroxysteroids. Since $17 \beta$-hydroxysteroids (T and E2) have higher affinity for the receptors than the keto-steroids (A4 and E1), this balance determines the final androgenic/estrogenic activity. Fourteen $17 \beta$ HSDs exist, whose specificity is determined by tissue distribution, intracellular localization and biochemistry (Table 2); reviewed thoroughly in (Mindnich et al., 2004; Moeller and Adamski, 2006, 2009; Prehn et al., 2009; Miller and Auchus, 2011). Unpublished data also refer to a 15th 17ßHSD (see Table 2; reported in Luu-The et al., 2008) with a putative role in androgen metabolism. With the exclusion of $17 \beta$ HSD 5 (AKR1C3, see below), all other $17 \beta$ HSDs belong to the shortchain dehydrogenase (SRD) family.

Although all $17 \beta$ HSDs have been postulated to use steroids as substrates based on cell-free or in vitro assays, recent investigations based on substrate specificity (Laplante et al., 2009) and knock-out (KO) models (Table 4) better clarified their roles. Type $117 \beta \mathrm{HSD}$ is the estrogenic enzyme and coverts E1 to E2 both in the ovary and in peripheral tissue. Type 2 $17 \beta$ HSD oxidizes 17-hydroxyl groups (E2 and T) to the 17-

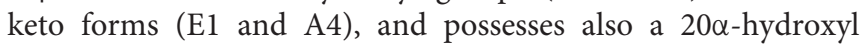
oxidative action, through which this enzyme generates $\mathrm{P}$ from $20 \alpha$ DHP. Type $617 \beta$ HSD uses $5 \alpha$-reduced androgens and has 17-hydroxyl oxidative activity (converting androsterone - AN- to androstanedione) and 3-hydroxyl oxidative activity (converting $3 \alpha \mathrm{DIOL}$ to the most potent androgen dihydrotestosterone DHT). Additional catalytic actions for 17 $\beta$ HSD6 (epimerase or 17-hydroxydehydrogenase) are demonstrated in vitro (Table 2). Type $1417 \beta$ HSD is postulated to have $17 \beta$-hydroxyl oxidative action on various steroids, type 7 is involved in cholesterol metabolism as indicated by KO mice (Table 4), whereas there is apparently little/no in vivo role of types 8, 9, 10, 11 and 12 $17 \beta$ HSDs on steroid metabolism (Table 2 and indicated by KO mice, Table 4). Recently, a novel SRD, DHRS11, was shown to possess in vitro 17 -keto to $17 \beta$-hydroxyl reductive action (able to use $\mathrm{E} 1, \Delta^{5}$ or $\Delta^{4}$ androstenes, androstanes), plus reductive $3 \beta$ HSD activity toward $\Delta^{4}$ pregnenes and other compounds (5 $\beta$-steroids, bile acids; Table 2 and Figure 2; Endo et al., 2016).

Androgens and progestogens can be further metabolized by aldo-ketoreductases (AKRs) and $5 \alpha$-reductases (SRD5As; Figure 2). Cytoplasmic AKRs (AKR1C1, 1C2, 1C3/17ßHSD5 and 1C4) have broad substrate specificity with non-stereo-selective $3 \alpha / 3 \beta$ HSD, 17- and 20-ketosteroid reductase activities (Table 2; Penning et al., 2004; Steckelbroeck et al., 2010). Together with the fact that they have wide tissue distribution (only AKR1C4 is restricted), AKR1Cs contribute to make intracrine networks flexible and intricate (Rižner and Penning, 2014; Sinreih et al., 2014).

SRD5As convert 3-keto $\Delta^{4}$ androstene and pregnene to $5 \alpha$ reduced steroids (androstanes and pregnanes), hence they are important in progestogen, androgen (DHT production) and neurosteroid metabolism (Di Costanzo et al., 2009). SRD5A1 and 3 are widely expressed, in contrast to SRD5A2. Human $5 \beta$ reductase activity, catalyzed by AKR1D1, is restricted to the liver, where $5 \beta$-steroids are directed to clearance/catabolism. However, some $5 \beta$-compounds are neuroactive and recent studies indicate 


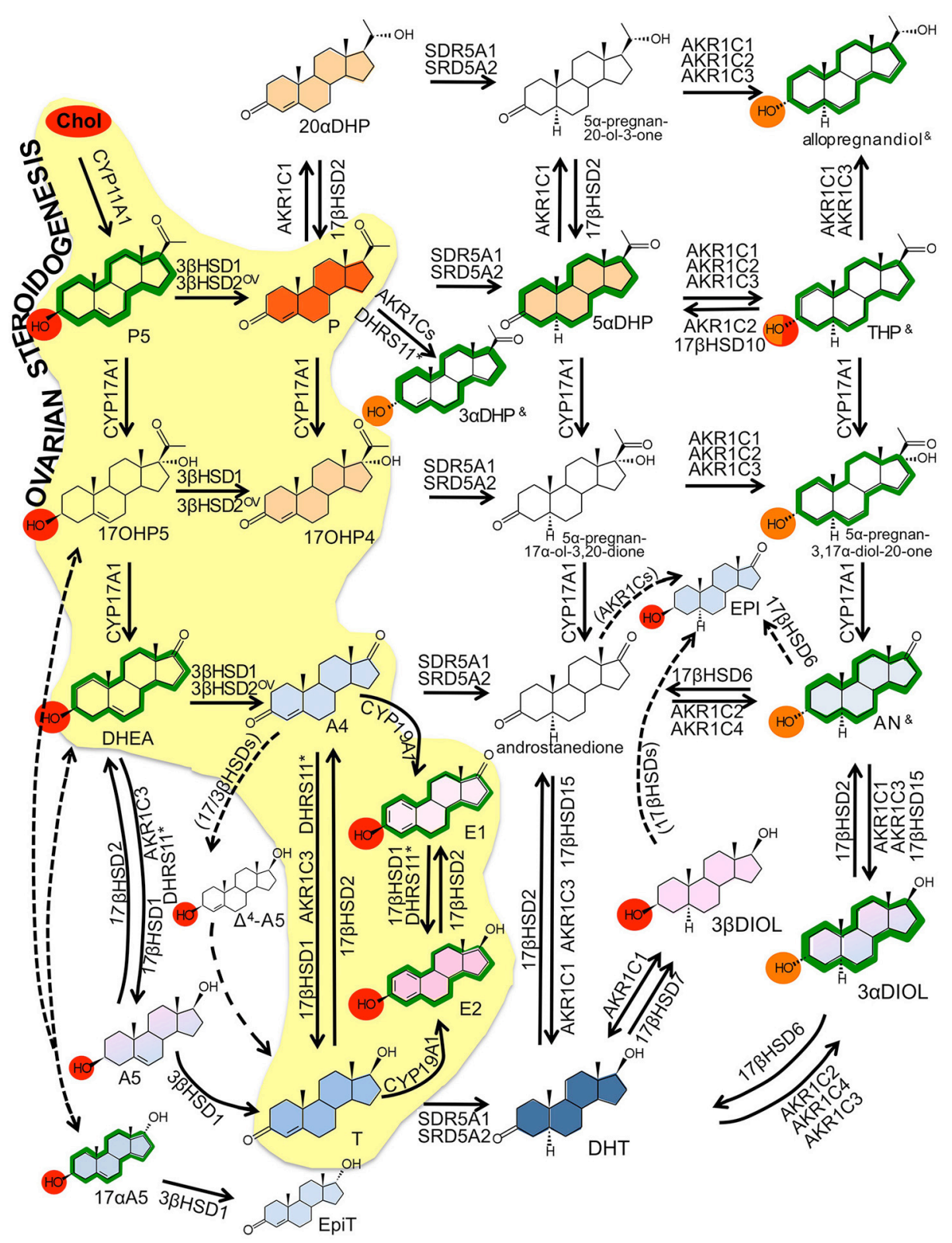

FIGURE 2 | Intracrine networks. Major intracrine networks metabolizing steroids. In this figure, each reaction reports the catalyzing enzymes whose role in that specific reaction is established based on robust evidences (in vitro, ex vivo, in vivo). Additional enzymes whose involvement in the same reactions is less robustly demonstrated or based only on in silico or cell-free assay are reported in Table 2 . The role of $17 \beta$ HSD3 is disregarded in this figure because restricted to tissues that are not assessed in the present review (testes, prostate, Table 2).

Color codes:

Yellow Background: Ovarian classic steroidogenesis.

Red circles: $3 \beta$-sulphated hydroxyl groups.

Orange circles: $3 \alpha$-sulphated hydroxyl groups.

\begin{tabular}{|cl|}
\hline $\begin{array}{l}\text { Biological activity } \\
\text { weak strong }\end{array}$ & progesterone receptor activation (PRA/B) \\
& androgen receptor activation (AR) \\
& estrogen receptor activation $(\mathrm{ER} \alpha / \beta)$ \\
& $\mathrm{GABA}_{\mathrm{A}}$ receptor allosteric modulator \\
&
\end{tabular}

OV ovarian specific referring to $3 \beta-H S D 2$ (see text); - dotted arrows indicate reactions that are not fully demonstrated to occur or for which the responsible enzyme is not identified yet; (enzyme name) enzymes indicated by brackets are supposed to catalyze the indicated reaction based on the theoretical assumptions, no experimental proof is yet available; \& these compounds (THP, $3 \alpha \mathrm{DHP}$ and allopregnandiol) exist as various hydroxyl $\alpha / \beta$ isomers (3, 5 , 17 ) with no activity, classic action or neuroactivity (see Table 2); * the role of DHRS11 in steroid metabolism is reported only recently by one publication (Endo et al., 2016). 
TABLE 1 | Major steroidal compounds.

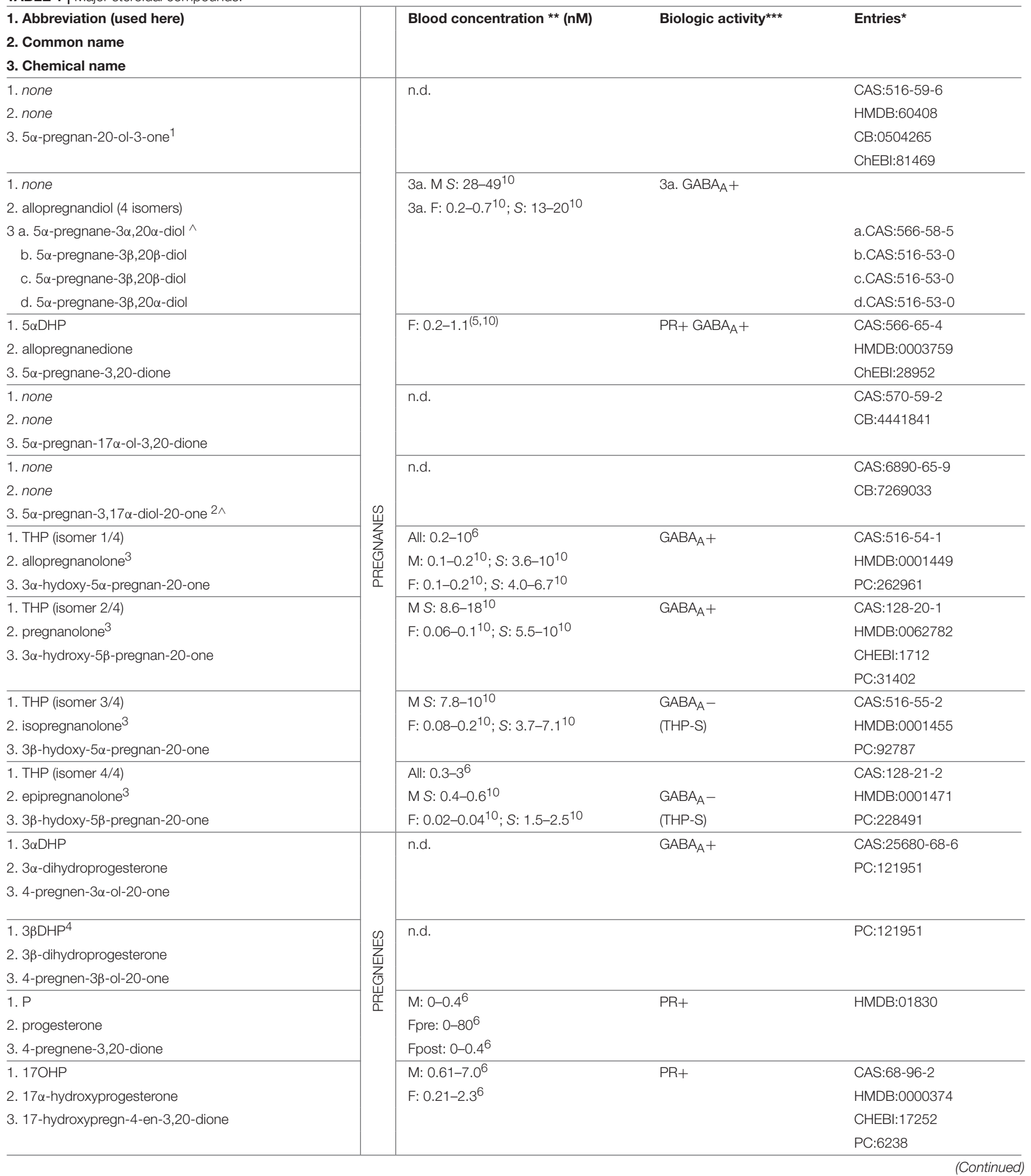


TABLE 1 | Continued

\begin{tabular}{|c|c|c|c|c|}
\hline $\begin{array}{l}\text { 1. Abbreviation (used here) } \\
\text { 2. Common name } \\
\text { 3. Chemical name }\end{array}$ & & Blood concentration ** $(\mathrm{nM})$ & Biologic activity*** & Entries* \\
\hline $\begin{array}{l}\text { 1. } 20 \alpha \mathrm{DHP} \\
\text { 2. } 20 \alpha \text {-dihydroprogesterone } \\
\text { 3. } 20 \alpha \text {-hydroxypreg-4-en-3-one }\end{array}$ & \multirow{3}{*}{ 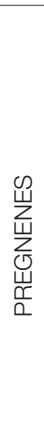 } & Fpre: $0.8-11.7^{6}$ & PR+ & $\begin{array}{l}\text { CAS:145-14-2 } \\
\text { HMDB:0003069 } \\
\text { PC:8956 }\end{array}$ \\
\hline $\begin{array}{l}\text { 1. } 17 \mathrm{OHP5} \\
\text { 2. 17-hydroxypregnenolone } \\
\text { 3. 5-Pregnen-3 } \beta, 17 \alpha \text {-diol-20-one }\end{array}$ & & $\begin{array}{l}\text { M: } 1.0-12^{6} \\
\text { F: } 0-6.0^{6}\end{array}$ & & $\begin{array}{l}\text { CAS:387-79-1 } \\
\text { HMDB:0000363 } \\
\text { CHEBI:28750 } \\
\text { PC:3032570 }\end{array}$ \\
\hline $\begin{array}{l}\text { 1. P5 } \\
\text { 2. pregnenolone } \\
\text { 3. pregn-5-en-3 } \beta \text {-ol-20-one }\end{array}$ & & $\begin{array}{l}\text { M: } 1-15^{6} ; S: 200-1,000^{6} \\
\text { Fpre: } 1.0-15^{6} ; S: 100-1,000^{6} \\
\text { Fpost: } 1.0-15^{6} ; S: 10-500^{6}\end{array}$ & $\mathrm{GABA}_{A}-(\mathrm{P} 5-\mathrm{S})$ & $\begin{array}{l}\text { CAS:145-13-1 } \\
\text { HMDB:0000253 } \\
\text { CHEBI:16581 } \\
\text { PC:8955 }\end{array}$ \\
\hline $\begin{array}{l}\text { 1. } 5 \beta A N \\
\text { 2. etiocholanolone } \\
\text { 3. } 3 \alpha \text {-hydroxy- } 5 \beta \text {-androstan-17-one }\end{array}$ & \multirow{3}{*}{ 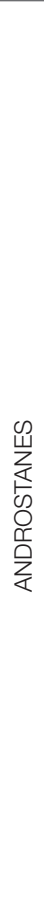 } & $\begin{array}{l}\text { M: } 0.09-0.2^{10} ; S: 32-70^{10} \\
F: 0.1-0.4^{10} ; S: 34-88^{10}\end{array}$ & $\mathrm{GABA}_{\mathrm{A}}+$ & $\begin{array}{l}\text { CAS:53-42-9 } \\
\text { HMDB:00490 } \\
\text { CHEBI: } 28195 \\
\text { PC:5880 }\end{array}$ \\
\hline $\begin{array}{l}\text { 1. } 3 \alpha \mathrm{DIOL} \\
\text { 2. androstanediol } \\
\text { 3. } 5 \alpha \text {-androstane- } 3 \alpha, 17 \beta \text {-diol }\end{array}$ & & $\begin{array}{l}\text { M: } 0.4-0.5^{9,10} ; S: 35-121^{10} \\
F: 0.03-0.06^{10} ; S: 2.4-4.8^{10}\end{array}$ & $\begin{array}{l}\mathrm{ER} \beta+\text { weak } \\
\mathrm{GABA}_{A}+\end{array}$ & HMDB:0000495 \\
\hline 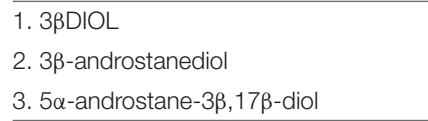 & & $\begin{array}{l}\text { All: } 0.15^{7} \\
\text { M S: } 34-103^{10} \\
\text { F S: } 8.7-18^{10}\end{array}$ & $\mathrm{ER} \beta+$ & HMDB:0000493 \\
\hline $\begin{array}{l}\text { 1. Epi }{ }^{8} \\
\text { 2. epitestosterone } \\
\text { 3. } 17 \alpha \text {-hydroxy-4-androsten-3-one }\end{array}$ & \multirow{5}{*}{ 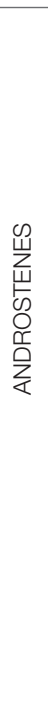 } & M: $1.3-2.9^{7}$ & $A R+$ weak9 & $\begin{array}{l}\text { CAS:481-30-1 } \\
\text { HMDB:0000628 } \\
\text { CHEBI:42534 } \\
\text { CB:10204 }\end{array}$ \\
\hline $\begin{array}{l}\text { 1. A5 } \\
\text { 2. androstenediol } \\
\text { 3. androst-5-ene- } 3 \beta, 17 \beta \text {-diol }\end{array}$ & & $\begin{array}{l}\text { M: } 2.6-3.7^{9,10} ; S: 243-494^{10} \\
F: 0.8-1.1^{10,11} ; S: 85-302^{10}\end{array}$ & AR+ weak & $\begin{array}{l}\text { CAS 521-17-5 } \\
\text { HMDB:0003818 } \\
\text { CHEBI: } 2710 \\
\text { PC: } 10634\end{array}$ \\
\hline $\begin{array}{l}\text { 1. } 17 \alpha \mathrm{A} 5 \\
\text { 2. } 17 \alpha \text { - androstenediol } \\
\text { 3. androst-5-ene-3 } \beta, 17 \alpha \text {-diol }\end{array}$ & & n.d. & $\begin{array}{l}\text { AR+ weak } \\
\text { GAB A } A^{-}\end{array}$ & $\begin{array}{l}\text { CAS:521-17-5 } \\
\text { HMDB:0003818 } \\
\text { CHEBI: } 2710 \\
\text { PC: } 10634\end{array}$ \\
\hline $\begin{array}{l}\text { 1. } \Delta^{4} \text {-A5 } \\
\text { 2. 4-androstenediol } \\
\text { 3. androst-4-ene-3 } 3,17 \beta \text {-diol }\end{array}$ & & n.d. & & $\begin{array}{l}\text { CAS:1156-92-9 } \\
\text { HMDB:0005849 } \\
\text { PC: } 12476620\end{array}$ \\
\hline $\begin{array}{l}\text { 1. DHEA } \\
\text { 2. dehydroepiandrosterone } \\
\text { 3. (3ß)-3-hydroxyandrost-5-en-17-one }\end{array}$ & & $\begin{array}{l}\text { M: } 10-25^{6} ; S: 2 \mathrm{~K}-10 \mathrm{~K}^{6} \\
\text { Fpre: } 3.0-30^{6} ; \text { S: } 1 \mathrm{~K}-8 \mathrm{~K}^{6} \\
\text { Fpost: } 2.0-20^{6} ; \text { S: } 1 \mathrm{~K}-6 \mathrm{~K}^{6}\end{array}$ & $\begin{array}{l}\mathrm{GABA}_{A-} \\
\text { (DHEA and DHEA-S) }\end{array}$ & HMDB:0000077 \\
\hline
\end{tabular}


TABLE 1 | Continued

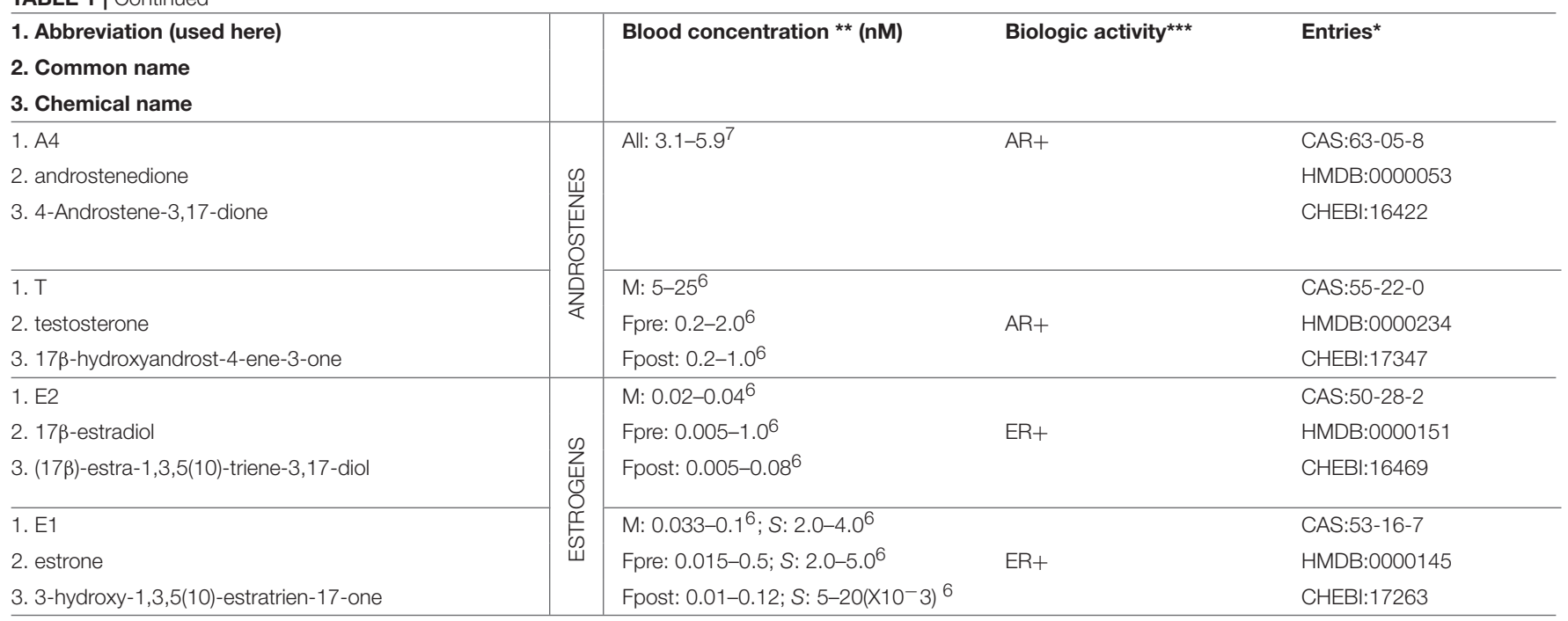

List of the steroids discussed in the present review with the major features. Nomenclature of these compounds is variable and aliases are given as Supplemental Materials.

"CAS: Chemical Abstracts Service, a division of the American Chemical Society (www.cas.org. Accessed on date: February 2018); HMDB: Human Metabolome Data Base (www.hmdb.

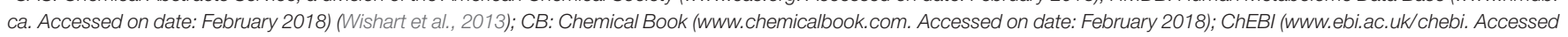
on date: February 2018) (Morgat et al., 2015); PC: PubChem (www.ncbi.nlm.nih.gov; pubchem.ncbi.nlm.nih.gov. Accessed on date: February 2018) (Kim et al., 2016).

${ }^{* *}$ M: male subjects; F: female subjects; Fpre: female premenopausal subjects; Fpost: female postmenopausal subjects; -S: sulphated compounds.

${ }^{* * *} P R, E R, A R$ : compound activates the indicated steroid receptor; GABA $A$ allosteric positive (+) or negative (-) modulator.

$\wedge$ This isomer is shown in Figure 2.

n.d.: not determined.

1 20 $\beta$-/5 $\beta$-isomers exist (CB8678436, Chemical Book - www.chemicalbook.com. Accessed on date: February 2018); ${ }^{2}$ Isomer 5 $\alpha$-pregnan-3 $\beta, 17 \alpha$-diol-20-one exists (CB:0291774, Chemical Book - www.chemicalbook.com. Accessed on date: February 2018); ${ }^{3}$ In general, $5 \alpha$-reduced and $3 \alpha$-hydroxysteroids are positive allosteric modulator of GABA $A$, whereas $3 \alpha$ - and $3 \beta$-sulphated hydroxysteroids and $5 \beta$-reduced steroids are negative allosteric modulator of GABA $A$. The $3 \beta$-hydroxy isomers of THP are inactive (Belelli and Lambert, 2005; Gibbs et al., 2006; ${ }^{4}$ This compound is GABA receptor inactive; ${ }^{5}$ Pearson Murphy et al., 2001; ${ }^{6}$ Mueller et al., 2015), ${ }^{7}$ Data extracted from the Human Metabolome Data Base-HMDB (www.hmdb.ca. Accessed on date: February 2018) (Wishart et al., 2013); ${ }^{8}$ This compound is strong inhibitor of SRD5As. ${ }^{9}$ Kancheva et al. (2007) and ${ }^{10}$ Bicikova et al. (2013).

the presence of AKR1D1 in placenta and myometrium (Jin et al., 2011). With the exclusion of their neuroactivity (Paragraph 4.6), $5 \beta$-steroids will not be further considered.

The sulphatase pathway is finally responsible for the balance between sulpho-conjugated and free steroids. Sulpho-conjugated steroids (-S) possess higher water solubility, increased stability and longer half-life than unconjugated compounds (e.g., 10$12 \mathrm{~h}$ vs. 20-30 min for estrogens), and although they cannot bind steroid-receptors, they serve as a reservoir for the formation of biologically active steroids (Reed et al., 2005). Sulphotransferases (SULTs) are phase-I detoxifying enzymes that use bis-phosphonucleotide $3^{\prime}$-phospho-adenosine- $5^{\prime}$-phosphate- (PAP)-sulfate as donor to conjugate $3 \beta$-hydroxyl steroids (e.g., estrogens, DHEA, P5, cholesterol; red circles in Figure 2) with a sulfate group (Strott, 2002; Rižner, 2016). Distinct SULTs have different specificities toward substrates, with SULT1E1 being the major estrogen sulphating enzyme (with little contribution of SULT1A1), and SULT2A1 being specific for DHEA (but also for P5, 17OHP5 and A5) (Table 2). Steroid sulphatase (STS) is a membrane-bound microsomal enzyme that catalyzes the hydrolysis of sulfate ester bonds from sulphated-steroids (cholesterol-S, P5-S, 17OHP5-S, DHEA-S, E1-S) (Mueller et al., 2015; Rižner, 2016), thus releasing unconjugated compounds.

Although sulphated-3 $\alpha$-hydroxysteroids are not thoroughly

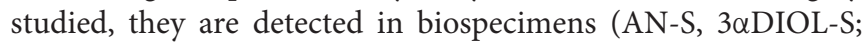
Table 1 and orange circles in Figure 2). They are most likely produced by SULT2A1 (active on $3 \alpha$-hydroxy bile acids) (Strott, 2002; Rižner, 2016) but no $3 \alpha$-stereo specific sulphatase is known to date. Some intracellular sulphated-steroids are converted to other compounds without prior desulphation (Sánchez-Guijo et al., 2016).

In conclusions, intracrinology presents redundant and complex pathways, which generate compounds with various activities. Genetic variants in intracrine genes are associated with various diseases (classically endocrine and not; Table 5). Even in the absence of the enzymatic machinery to metabolize cholesterol (StAR, steroidogenic factor, CYP17A1 and CYP11A1), DHEA, P5 and especially their sulphated-conjugates have high blood concentrations (Table 1), and are used to generate all other steroids in peripheral tissues.

\section{DRUG DEVELOPMENT}

Natural hormones have been historically used as drugs, and depending on definitions, approximately 90 marketed drugs share a steroidal core (see https://www.drugbank. ca). Steroids (T, E2, cortisol, DHEA), simple derivatives (ethinylestrogen, prednisolone) or more complex analogs (abiraterone, fulvestrant) are used in various conditions. This old-and-proven steroidal chemistry based approach is used even in modern era. 
TABLE 2 | Major enzymes involved in steroidogenesis.

\begin{tabular}{|c|c|c|c|c|}
\hline & Chromosome & Protein $(\mathrm{aa})^{\star \star}$ & & \\
\hline 1. Abbr. & Gene size* & Dalton & 1. Name & 2. Family \\
\hline 2. Gene ID & mRNA size* & Localization (L) $)^{\star \star \star}$ & 3. Catalysis & 4. Substrates ${ }^{\star \star \star \star}$ \\
\hline 3. Gene name(s) & Exons (no) & & 5. Distribution & 6. Cofactor \\
\hline 1. StAR & Chr: $8 p 11.23$ & & \multicolumn{2}{|c|}{ 1. steroidogenic acute regulatory protein } \\
\hline 2. 6770 & gene: 8.6 & aa: 285 & \multicolumn{2}{|c|}{ 2. cytochrome P450 } \\
\hline 3. STAR, STARD1 & mRNA: 1.6 & Dalton: 31,914 & \multicolumn{2}{|c|}{ 3. facilitate transport of cholesterol to mitochondria } \\
\hline & Exons: 8 & L: mitochon & \multicolumn{2}{|l|}{ 4. cholesterol } \\
\hline & & & \multicolumn{2}{|c|}{ 5. restricted (adrenal, testis, ovary, placenta) } \\
\hline
\end{tabular}

\section{CYP11A1}

2. 1583

3. CYP11A1;

CYP11A;

CYPXIA1;

P450SCC

1. CYP17A1

2. 1586

3. CYP17A1; CPT7;

CYP17; S17AH;

P450C17
Chr: $15 q 24.1$

Gene: 30.0

mRNA: 2.0

Exons: 9
1. cytochrome $P 450$ side-chain cleavage enzyme

2. cytochrome P450, type I

3. cleavage of cholesterol side-chain

4. cholesterol $\rightarrow$ P5

5. restricted (adrenal, testis, ovary, placenta)

6. NADP/NADPH
1. steroid $17 \alpha$-hydroxylase/17,20-lyase

2. cytochrome P450, type II

3. 17 $\alpha$-hydroxylase and 17,20-lyase activities

4. $\mathrm{P} 5 \rightarrow \mathrm{DHEA} ; \mathrm{P} \rightarrow \mathrm{A} 4{ }^{\$} ; 5 \alpha \mathrm{DHP} \rightarrow$ androstanedione; THP $\rightarrow$ 5 $\alpha$-pregnan-3,17 $\alpha$-diol-20-one

5. restricted (adrenal, testis, ovary, placenta)

6. NADP/NADPH

\section{CYP19A1 \\ 2. 1588 \\ 3. CYP19A1; ARO; \\ ARO1; CPV1; CYAR; \\ CYP19; CYPXIX;}

$P-450 A R O M$
Chr: $10 \mathrm{q} 24.32$

mRNA: 1.9 Dalton: 57,37

Exons: $8 \quad$ L: EndRet
1. cytochrome $P 450$ aromatase

2. cytochrome P450, type II

3. oxidative demethylation of $C_{19}$ to $C_{18}$ (aromatisation)

4. $\mathrm{A} \rightarrow \mathrm{E} 1 ; \mathrm{T} \rightarrow \mathrm{E} 2$

5. restricted (adrenal, testis, ovary, placenta)

6. NADP/NADPH
1. $3 \beta \mathrm{HSD} 1$
2. 3283
Chr: 1p11-12
3. $H S D 3 B 1 ; H S D 3 B$;
Gene: 8.1
1. $3 \beta$-hydroxysteroid dehydrogenase/ $\Delta^{5 \rightarrow 4}$ isomerase type I
HSDB3; SDB3A;
mRNA: 1.7
aa: 373
Exons: 4
Dalton: 42,252
2. short chain dehydrogenase/reductase superfamily (more ${ }^{\wedge \wedge}$ )
3. oxidative conversion of $\Delta^{5} 3 \beta$-hydroxyl to $\Delta^{4}$ keto-steroids
4. $\mathrm{P} 5 \rightarrow \mathrm{P} ; 17 \mathrm{OHP} 5 \rightarrow$ 17OHP4; DHEA $\rightarrow$ A4; A5 $\rightarrow$ T; 17aA5 $\rightarrow$ EpiT
5. selectively distributed (placenta, periphery)
6. NADP/NADPH or NAD/NADH

aa: 503

mRNA: $1.5-4.5$

L: EndRet
1. $3 \beta \mathrm{HSD} 2$
2. 3284
Chr: 1p11-13
3. HSD3B2; HSDB; Gene: 8.1 HSD3B; SDR11E2
mRNA: 1.7
Exons: 4
aa: 372
1. $3 \beta$-hydroxysteroid dehydrogenase/ $\Delta^{5: 4}$ isomerase type II
2. short chain dehydrogenase/reductase superfamily
Dalton: 42,052
3. oxidative conversion of $\Delta^{5}$-3 $\beta$-hydroxyl to $\Delta^{4}$-ketosteroids
4. $\mathrm{P} 5 \rightarrow \mathrm{P} ; 17 \mathrm{OHP} 5 \rightarrow$ 17OHP4; $\mathrm{DHEA} \rightarrow \mathrm{A} 4$
5. restricted (adrenal, testis, ovary)
6. NADP/NADPH or NAD/NADH

\begin{tabular}{lll}
\hline 1. $17 \beta$ HSD1 & Chr: $17 q 11-21$ & \\
2. 3,292 & Gene: 6.0 & aa: 328 \\
3. $H S D 17 B 1$ & mRNA: $1-2.4$ & Dalton: 34,950 \\
E2DH; HSD17; & Exons: 6 & L: cytoplasm \\
$\begin{array}{l}\text { EDHB17; } E D H 17 B 2 ; \\
\text { SDR28C1; }\left(\text { more }^{\wedge \wedge}\right)\end{array}$ & &
\end{tabular}

1. $17 \beta$-hydroxysteroid dehydrogenase type 1

2. short chain dehydrogenase/reductase superfamily

3. reduction of 17 -keto to $17 \beta$-hydroxyl (estrogens)

4. (established) $\mathrm{E} 1 \rightarrow \mathrm{E} 2 ;\left(\mathrm{A} 4 \rightarrow \mathrm{T}\right.$ in rodents) (postulated) $\mathrm{DHEA} \rightarrow \mathrm{A} 5^{1} ; \mathrm{P} \rightarrow 20 \alpha \mathrm{DHP} P^{2}$; $\mathrm{DHT} \rightarrow 3 \beta \mathrm{DIOL}^{11} ; \mathrm{DHT} \rightarrow$ androstanedione ${ }^{11}$

5. selectively distributed (ovary, placenta (low in endometrium, breast) ${ }^{14,15}$

6. NADP/NADPH
1. $17 \beta \mathrm{HSD} 2$
2. 3294
Chr: 16q24.1-2
$\begin{array}{ll}\text { Gene: } 63 & \text { aa: } 387 \\ \text { mRNA: } 1.5 & \text { Dalton: } 42,785\end{array}$
1. 17ß-hydroxysteroid dehydrogenase type 2
3. HSD17B2 HSD17;
SDR9C2; EDH17B2
2. short chain dehydrogenase/reductase superfamily
3. oxidation of $17 \beta$-hydroxyl to 17 -keto (estrogens \& androgens)

(Continued) 
TABLE 2 | Continued

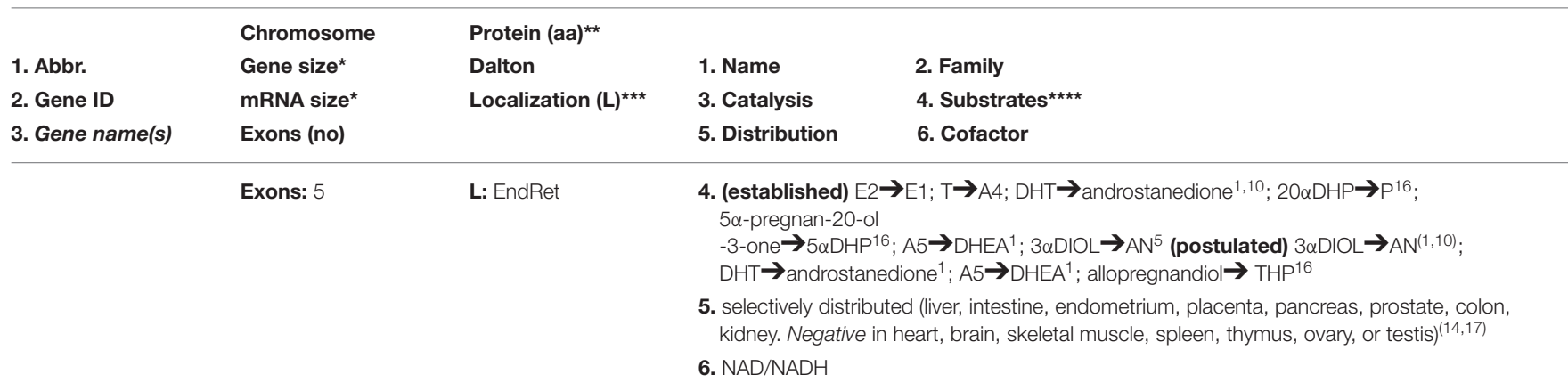

\begin{tabular}{|c|c|c|c|}
\hline 1. $17 \beta \mathrm{HSD} 3^{\& \&}$ & Chr: 9q22 & & 1. $17 \beta$-hydroxysteroid dehydrogenase type 3 \\
\hline 2. 3293 & Gene: 67 & aa: 310 & 2. short chain dehydrogenase/reductase superfamily \\
\hline 3. $H S D 17 B 3$ & mRNA: 1.2 & Dalton: 34,516 & 3. reduction of 17-keto to $17 \beta$-hydroxyl (androgens) \\
\hline
\end{tabular}

EDH17B3;

Exons: $11 \quad$ L: EndRet 4. (established) $A 4 \rightarrow T$

(postulated) $\mathrm{AN} \rightarrow 3 \alpha \mathrm{DIOL}^{(1,3,10)}$; androstanedione $\rightarrow \mathrm{DHT}^{(1,9,10)}$

5. restricted (testis; low in brain, blood, skin, adipose tissue) ${ }^{14}$

6. NADP/NADPH

\begin{tabular}{|c|c|}
\hline 1. $17 \beta \mathrm{HSD} 4$ & Chr: $5 q 23.1$ \\
\hline 2. 3295 & Gene: 184 \\
\hline 3. HSD17B4 & mRNA: 2.9 \\
\hline $\begin{array}{l}\text { DBP; MFE-2; MPF-2; } \\
\text { PRLTS1; SDR8C1 }\end{array}$ & Exons: 28 \\
\hline
\end{tabular}

aa: $736 \quad$ 1. $17 \beta$-hydroxysteroid dehydrogenase type 4

Dalton: $79,686 \quad$ 2. short chain dehydrogenase/reductase superfamily

L: Peroxisome mitochon 3. fatty acid $\beta$-oxidation (steroids in pigs)

PRLTS1; SDR8C1 4. (established) very long chain branched fatty acids, bile acids
(postulated) $A 5 \rightarrow D H E A^{1} ; E 2 \rightarrow E 1^{14}$

5. ubiquitous (liver, heart, prostate, testis, lung, skeletal muscle, kidney, pancreas, thymus, ovary, intestine, placenta, brain, spleen, colon, lymphocytes) ${ }^{14}$

6. NAD/NADH

\begin{tabular}{|c|c|c|c|}
\hline 1. $17 \beta \mathrm{HSD} 6$ & Chr: $12 q 13$ & aa: 317 & 1. $17 \beta$-hydroxysteroid dehydrogenase type 6 \\
\hline 2. 8630 & Gene: 24.5 & Dalton: 35,966 & 2. short chain dehydrogenase/reductase superfamily \\
\hline 3. HSD17B6 & mRNA: 1.6 & L: EndRet & 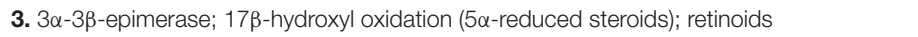 \\
\hline \multirow[t]{3}{*}{$\begin{array}{l}\text { HSE; RODH; } \\
\text { SDR9C6 }\end{array}$} & Exons: 8 & microsomes & $\begin{array}{l}\text { 4. (established) } 3 \alpha \mathrm{DIOL} \rightarrow \mathrm{DHT}^{10} ; \mathrm{AN} \rightarrow \text { androstanedione }^{5} ; \mathrm{AN} \rightarrow \mathrm{EPI} \\
\text { (postulated) } 3 \alpha \mathrm{DIOL} \rightarrow \mathrm{AN}^{5} ; \mathrm{E} 2 \rightarrow \mathrm{E} 1^{5}\end{array}$ \\
\hline & & & 5. selectively distributed (liver, testis, lung, spleen, brain, ovary, kidney, adrenal, prostate) ${ }^{14}$ \\
\hline & & & 6. NAD/NADP \\
\hline
\end{tabular}

\begin{tabular}{|c|c|c|c|}
\hline 1. $17 \beta \mathrm{HSD} 7$ & Chr: 1q23 & & 1. $17 \beta$-hydroxysteroid dehydrogenase type 7 \\
\hline 2. 51478 & Gene: 22.1 & aa: 341 & 2. short chain dehydrogenase/reductase superfamily \\
\hline 3. HSD17B7 & mRNA: 1.5 & Dalton: 38,206 & 3. 3-ketosteroid reductase of sterols \\
\hline \multirow[t]{3}{*}{ PRAP; SDR37C1 } & Exons: 9 & L: EndRet & 4. (established) Sterols/cholesterol biosynthesis; $\mathrm{DHT} \rightarrow 3 \beta \mathrm{DIOL}^{1}$ \\
\hline & & & $\begin{array}{l}\text { 5. widely distributed (ovary, uterus, placenta, liver, breast, testis, neuronal tissue, adrenal gland, } \\
\text { small intestine, prostate, adipose tissue lung, and thymus) }{ }^{(14,18)}\end{array}$ \\
\hline & & & 6. NADP/NADPH \\
\hline
\end{tabular}
1. $17 \beta$ HSD 8
Chr: $6 \mathrm{p} 21.3$
2. 7923
Gene: 2.2
aa: 261
1. $17 \beta$-hydroxysteroid dehydrogenase type 8
3. $H S D 17 B 8$
mRNA: 1.0
Dalton: 26,974
2. short chain dehydrogenase/reductase superfamily
KE6; FABG; HKE6;
Exons: 9
L: mitochon
3. fatty acid elongation; steroid $17 \beta \mathrm{HSD}$ action (rodents).
FABGL; RING2;
H2-KE6; $\left(\right.$ more $\left.^{\wedge \wedge}\right)$
4. (established) fatty acids (postulated) E2 $\rightarrow \mathrm{E} 1$
5. widely distributed (prostate, placenta, kidney, brain, cerebellum, heart, lung, small intestine, ovary, testis, adrenal, stomach, liver, adrenals) ${ }^{14-19}$
6. NAD/NADP

\begin{tabular}{|c|c|c|c|}
\hline 1. $17 \beta$ HSD9 & Chr: 12q23 & & 1. $17 \beta$-hydroxysteroid dehydrogenase type 9 \\
\hline 2. 5959 & Gene: 4.4 & aa: 318 & 2. short chain dehydrogenase/reductase superfamily \\
\hline 3. HSD17B9 & mRNA: 1.4 & Dalton: 34,979 & 3. retinoid metabolism (steroid metabolism in rodents) \\
\hline RDH5; (more $\left.{ }^{\wedge \wedge}\right)$ & Exons: 4 & L: EndRet & 4. (established) retinoids (postulated) $\mathrm{AN} \rightarrow 3 \alpha \mathrm{DIOL}$ \\
\hline 1. $17 \beta \mathrm{HSD} 10$ & Chr: Xp11.2 & & 1. $17 \beta$-hydroxysteroid dehydrogenase type 10 \\
\hline 2. 3028 & Gene: 3.1 & aa: 261 & 2. short chain dehydrogenase/reductase superfamily \\
\hline
\end{tabular}


TABLE 2 | Continued

\begin{tabular}{|c|c|c|c|c|}
\hline & Chromosome & Protein $(\mathrm{aa})^{\star \star \star}$ & & \\
\hline 1. Abbr. & Gene size* & Dalton & 1. Name & 2. Family \\
\hline 2. Gene ID & mRNA size* & Localization $(\mathbf{L})^{\star \star \star}$ & 3. Catalysis & 4. Substrates ${ }^{\star \star \star \star}$ \\
\hline 3. Gene name(s) & Exons (no) & & 5. Distribution & 6. Cofactor \\
\hline 3. HSD17B10 & mRNA: 0.9 & Dalton: 26,923 & \multicolumn{2}{|c|}{ 3. fatty acids \& steroid oxidation; tRNA maturation } \\
\hline $\begin{array}{l}\text { ABAD; CAMR; ERAB; } \\
\text { HCD2; MHBD; } \\
\text { HADH2; MRPP2; } \\
\text { MRX17; }\end{array}$ & Exons: 6 & L: mitochon & \multirow{2}{*}{\multicolumn{2}{|c|}{$\begin{array}{l}\text { 4. (established) Isoleucine, fatty acid, bile acid metabolism, } \mathrm{THP} \rightarrow 5 \alpha \mathrm{DHP}(20,34) \\
\text { (postulated) } 3 \alpha \mathrm{DIOL} \rightarrow \mathrm{AN}^{1,9} ; \mathrm{DHT} \rightarrow \text { androstanedione }{ }^{1,9} ; \mathrm{T} \rightarrow \mathrm{A}^{1} \\
\text { 5. nearly ubiquitous (liver, small intestine, colon, kidney, heart, brain, placenta, lung, ovary, testis, } \\
\text { spleen, thymus, prostate, leukocyte) }{ }^{14}\end{array}$}} \\
\hline MRX31; $\left(\right.$ more $\left.^{\wedge \wedge}\right)$ & & & & \\
\hline
\end{tabular}
1. $17 \beta \mathrm{HSD} 11$
Chr: 4q22.1
2. 51170
Gene: 54.9
mRNA: 1.9
aa: 300
1. $17 \beta$-hydroxysteroid dehydrogenase type 11
3. HSD17B11
Exons: 7
Dalton: 32,936
2. short chain dehydrogenase/reductase superfamily
DHRS8; PAN1B;
L: EndRet
3. Short-chain alcohol dehydrogenases
RETSDR2; SDR16C2;
$\left(\right.$ more $\left.{ }^{\wedge \wedge}\right)$
5. nearly ubiquitous (liver, intestine, kidney, adrenal gland, heart, lung, testis, ovary, placenta, sebaceous gland and pancreas) ${ }^{14,21}$
4. (established) lipids, sec. alcohols/ketones (postulated) $3 \alpha \mathrm{DIOL} \rightarrow \mathrm{AN}^{1,9}$
6. NAD/NADH

\begin{tabular}{|c|c|c|}
\hline 1. $17 \beta \mathrm{HSD} 12$ & Chr: 11p11.2 & \\
\hline 2. 51144 & Gene: 170.1 & aa: 312 \\
\hline 3. HSD17B12 & mRNA: 2.6 & Dalton: 34,324 \\
\hline KAR; SDR12C1 & Exons: 11 & L: EndRet \\
\hline
\end{tabular}

1. $17 \beta$-hydroxysteroid dehydrogenase type 12

2. short chain dehydrogenase/reductase superfamily

3. fatty acid elongation, steroid $17 \beta \mathrm{HSD}$ reductive action (rodents)

4. (established) branched/long chain fatty acids (postulated) $\mathrm{E} 1 \rightarrow \mathrm{E} 2$

5. ubiquitous (heart, skeletal muscle, liver, kidney, adrenal gland, testis, placenta, brain, pancreas, GIT, trachea, lung, thyroid, prostate, aorta, bladder, spleen, skin, ovary, breast, uterus, vagina) $)^{(14,22)}$

6. NADP/NADPH

\begin{tabular}{|c|c|c|c|}
\hline 1. $17 \beta \mathrm{HSD} 13$ & Chr: 4q22.1 & & 1. $17 \beta$-hydroxysteroid dehydrogenase type 13 \\
\hline 2. 345275 & Gene: 19.1 & aa: 300 & 2. short chain dehydrogenase/reductase superfamily \\
\hline 3. $H S D 17 B 13$ & mRNA: 2.3 & Dalton: 33,655 & 3. and 4. unknown \\
\hline $\begin{array}{l}\text { SCDR9; NIIL497; } \\
\text { (more }\end{array}$ & Exons: 6 & L: extracell/EndRet & $\begin{array}{l}\text { 5. restricted (liver; low in bone marrow, lung, ovary, testis, } \\
\text { kidney, skeletal muscle brain, bladder) }{ }^{14}\end{array}$ \\
\hline
\end{tabular}

\begin{tabular}{|c|c|c|c|}
\hline 1. $17 \beta \mathrm{HSD} 14$ & Chr: $19 q 13.33$ & & 1. $17 \beta$-hydroxysteroid dehydrogenase type 14 \\
\hline 2. 51171 & Gene: 23.7 & aa: 270 & 2. short chain dehydrogenase/reductase superfamily \\
\hline 3. HSD17B14 & mRNA: 1.3 & Dalton: 28,317 & 3. fatty acid \& prostaglandin metabolism; $17 \beta \mathrm{HSD}$ activity \\
\hline $\begin{array}{l}\text { DHRS10; SDR47C1; } \\
\text { retSDR3 }\end{array}$ & Exons: 8 & L: cytoplasm & $\begin{array}{l}\text { 4. (established) fatty acids } \\
\text { (postulated) } 3 \alpha \mathrm{DIOL} \rightarrow \mathrm{AN}^{1,9} ; \mathrm{E} 2 \rightarrow \mathrm{E} 1^{4} ; \mathrm{T} \rightarrow \mathrm{A} 4^{4} ; \mathrm{A} 5 \rightarrow \mathrm{DHEA}\end{array}$ \\
\hline & & & 5. widely distributed (brain, liver, placenta, breast) ${ }^{14}$ \\
\hline & & & 6. NAD/NADH \\
\hline
\end{tabular}

\begin{tabular}{|c|c|c|c|}
\hline 1. $17 \beta \mathrm{HSD} 15$ & Chr: $14 q 24.1$ & & 1. retinol dehydrogenase 11 \\
\hline 2. 51109 & Gene: 19.0 & aa: 318 & 2. short chain dehydrogenase/reductase superfamily \\
\hline \multirow{3}{*}{$\begin{array}{l}\text { 3. } R D H 11 ; \text { PSDR1; } \\
\left.\text { ARSDR1; (more }{ }^{\wedge \wedge}\right)\end{array}$} & mRNA: 1.8 & Dalton: 35,386 & 3. dehydrogenase activity of retinoid and steroids \\
\hline & Exons: 9 & L: cytoplasm & 4. (established) retinoids (post.) $\mathrm{AN} \rightarrow 3 \alpha \mathrm{DIOL}^{1,9}$; androstanedione $\rightarrow \mathrm{DHT}^{1,9}$ \\
\hline & & & $\begin{array}{l}\text { 5. widely distributed } \\
\text { 6. NADP/NADPH }\end{array}$ \\
\hline
\end{tabular}

\begin{tabular}{|c|c|c|c|}
\hline 1. $\mathrm{DHRS} 11^{23}$ & Chr: $17 q 12$ & & 1. dehydrogenase/reductase 11 \\
\hline 2. 79154 & Gene: 9.0 & aa: 260 & 2. short chain dehydrogenase/reductase superfamily \\
\hline 3. DHRS11 & mRNA: 1.6 & Dalton: 28,308 & 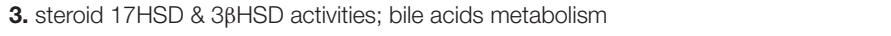 \\
\hline $\begin{array}{l}\text { ARPG836; SDR24C1; } \\
\text { spDHRS11 }\end{array}$ & Exons: 7 & L: extracell & $\begin{array}{l}\text { 4. } \mathrm{E} 1 \rightarrow \mathrm{E} 2 ; \mathrm{A} 4 \rightarrow \mathrm{T} \text {; DHEA } \rightarrow \text { A5; androstanedione } \rightarrow \mathrm{DHT} \text {; } \mathrm{AN} \rightarrow 3 \alpha \mathrm{DIOL} ; \mathrm{P} \rightarrow 3 \alpha \mathrm{DHP} \mathrm{P}^{23} \\
\text { 5. nearly ubiquitous (testis, small intestine, colon, kidney) }{ }^{23} \\
\text { 6. NADP/NADPH }\end{array}$ \\
\hline
\end{tabular}

\begin{tabular}{|c|c|c|c|}
\hline 1. $\mathrm{AKR} 1 \mathrm{C} 1$ & Chr: 10p14-15 & & 1. aldo-ketoreductase family 1 member $\mathrm{C} 1$ \\
\hline 2. 1645 & Gene: 20.0 & aa: 323 & 2. aldo-ketoreductase family \\
\hline 3. $A K R 1 C 1$ & mRNA: 12 & Dalton: 36,788 & $\begin{array}{l}\text { 3. } 20 \alpha \mathrm{HSD} \text { (strong) and } 17 \beta \mathrm{HSD} \text { (weak) activities; moderate 3-keto reduction to } 3 \beta \text {-hydroxyl } \\
(>3 \alpha)\end{array}$ \\
\hline
\end{tabular}


TABLE 2 | Continued

\begin{tabular}{|c|c|c|c|}
\hline & Chromosome & Protein $(\mathrm{aa})^{\star \star}$ & \\
\hline 1. Abbr. & Gene size* & Dalton & 2. Family \\
\hline 2. Gene ID & mRNA size* & Localization $(\mathbf{L})^{\star \star *}$ & 4. Substrates ${ }^{\star \star \star *}$ \\
\hline 3. Gene name(s) & Exons (no) & & 5. Distribution \\
\hline \multirow[t]{2}{*}{$\begin{array}{l}\text { C9, DDH, DDH1, DD1, } \\
\text { H-37, HBAB, MBAB } \\
\text { HAKRC; DD1/DD2; } \\
\text { 2-ALPHA-HSD; } \\
\text { 20-ALPHA-HSD }\end{array}$} & Exons: 9 & L: cytoplasm & 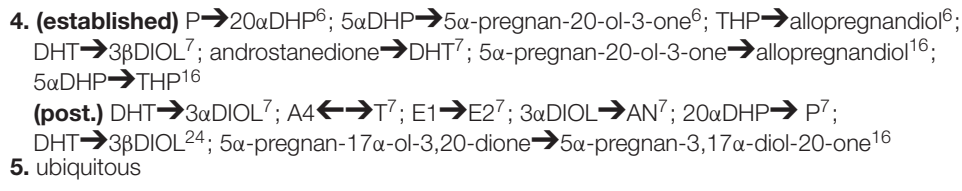 \\
\hline & & & 6. NADP/NADPH or NAD/NADH \\
\hline
\end{tabular}

\begin{tabular}{|c|c|c|c|}
\hline 1. AKR1C2 & Chr: $10 p 14-15$ & & 1. aldo-ketoreductase family 1 member C2 \\
\hline 2. 1646 & Gene: 30.6 & aa: 323 & 2. aldo-ketoreductase family \\
\hline $\begin{array}{l}\text { 3. AKR1C2 } \\
\text { DD; DD2; TDD; } \\
\text { BABP; DD-2; DDH2; } \\
\text { HBAB; HAKRD; } \\
\text { MCDR2; SRXY8; } \\
\text { DD/BABP; } \\
\text { AKR1C-pseudo }\end{array}$ & $\begin{array}{l}\text { mRNA: } 1.3 \\
\text { Exons: } 9\end{array}$ & $\begin{array}{l}\text { Dalton: } 36,735 \\
\text { L: cytoplasm }\end{array}$ & 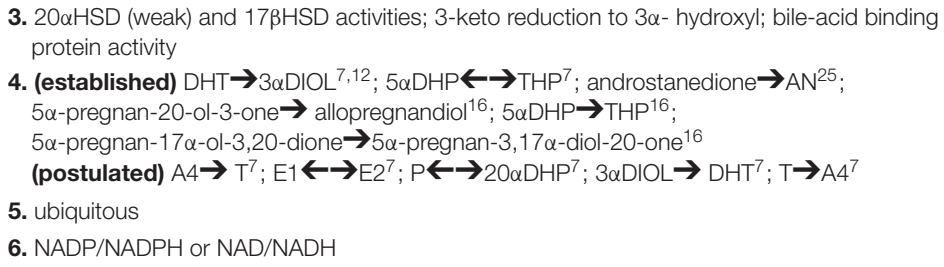 \\
\hline
\end{tabular}

\begin{tabular}{lll}
\hline 1. AKR1C3 & Chr: 10p14-15 & \\
2. 8644 & Gene: 13 & aa: 323 \\
3. HSD17B5 & mRNA: 1.2 & Dalton: 36,853 \\
AKR1C3 & Exons: 9 & L: cytoplasm \\
DD3; DDX; PGFS; & & \\
HAKRB; HAKRe; & & \\
HA1753; HSD17B5; & & \\
hluPGFS &
\end{tabular}

1. aldo-ketoreductase family 1 member C3

2. aldo-ketoreductase family

3. $20 \alpha$ HSD (weak) and $17 \beta$ HSD activities (androgens); 3-keto reduction to $3 \alpha$-/ $\beta$-hydroxyl (weak); 11-ketoprostaglandin reductase ${ }^{3}$

4. (established) $\mathrm{A} 4 \rightarrow \mathrm{T}^{7} ; \mathrm{DHT} \rightarrow 3 \alpha \mathrm{DIOL}^{7} ; 3 \alpha \mathrm{DIOL} \rightarrow \mathrm{AN}^{7} ; 5 \alpha \mathrm{DHP} \rightarrow \mathrm{THP}^{7}$; $5 \alpha$-pregnan-20-ol-3-one $\rightarrow$ allopregnandiol $^{16} ; \quad 5 \alpha \mathrm{DHP} \rightarrow \mathrm{THP}^{16} ; \mathrm{DHEA}^{\rightarrow} \mathrm{A}^{7}$; $5 \alpha$-pregnan-17 $\alpha$-ol-3,20-dione $\rightarrow 5 \alpha$-pregnan-3,17 $\alpha$-diol-20-one ${ }^{16}$; androstanedione $\rightarrow \mathrm{DHT}^{24}$ (postulated) $\mathrm{E} 1 \leftarrow \rightarrow \mathrm{E} 2^{7} ; \mathrm{T} \rightarrow \mathrm{A}^{7} ; 20 \alpha \mathrm{DHP} \leftarrow \rightarrow \mathrm{P}^{7}$

5. nearly ubiquitous (prostate, mammary gland, liver, kidney, lung, heart, uterus, testis, brain, skeletal muscle, adipose tissue, pancreas, hearth, skeletal muscle, thymus, ovary, small intestine and colon) ${ }^{14,26}$

6. NADP/NADPH or NAD/NADH

\begin{tabular}{|c|c|c|c|}
\hline 1. AKR1C4 & Chr: 10p15.1 & & 1. aldo-ketoreductase family 1 member $\mathrm{C} 4$ \\
\hline 2. 1109 & Gene: 25.2 & aa: 323 & 2. aldo-ketoreductase family \\
\hline 3. $A K R 1 C 4$ & mRNA: 1.2 & Dalton: 37,067 & 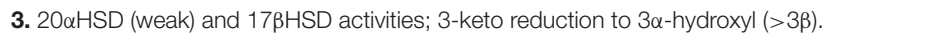 \\
\hline $\begin{array}{l}\text { C11; CDR; DD4; } \\
\text { CHDR; DD-4; } \\
\text { HAKRA; }\end{array}$ & Exons: 9 & L: cytoplasm & 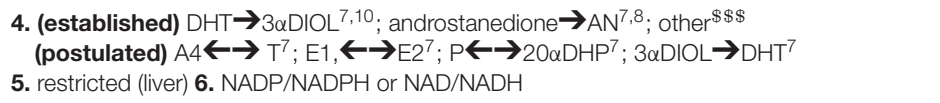 \\
\hline $\begin{array}{l}\text { 1. SRD5A1 } \\
\text { 2. } 6715 \\
\text { 3. SRD5A1 } \\
\text { S5AR } 1\end{array}$ & $\begin{array}{l}\text { Chr: } 5 p 15.31 \\
\text { Gene: } 41.0 \\
\text { mRNA: } 2.3 \\
\text { Exons: } 5(7)\end{array}$ & $\begin{array}{l}\text { aa: } 259 \\
\text { Dalton: } 29,459 \\
\text { L: EndRet }\end{array}$ & $\begin{array}{l}\text { 1. steroid } 5 \alpha \text {-reductase } 1 \text { 2. steroid } 5 \alpha \text { reductase family } \\
\text { 3. androgen and pregnene metabolism } \\
\text { 4. } \mathrm{T} \rightarrow \mathrm{DHT} ; \mathrm{A} 4 \rightarrow \text { androstanedione; } 17 \mathrm{OHP} 4 \rightarrow 5 \alpha \text {-pregnan-17 } \alpha \text {-ol-3,20-dione; } \mathrm{P} \rightarrow 5 \alpha \mathrm{DHP} \text {; } \\
20 \alpha \mathrm{DHP} \rightarrow 5 \alpha \text {-pregnan-20-ol-3-one } \mathbf{5} \text {. ubiquitous }\end{array}$ \\
\hline $\begin{array}{l}\text { 1. SRD5A2 } \\
\text { 2. } 6716 \\
\text { 3. SRD5A2 }\end{array}$ & $\begin{array}{l}\text { Chr: } 2 \mathrm{p} 23.1 \\
\text { Gene: } 178.3 \\
\text { mRNA: } 2.5 \\
\text { Exons: } 5\end{array}$ & $\begin{array}{l}\text { aa: } 254 \\
\text { Dalton: } 28,393 \\
\text { L: microsomes }\end{array}$ & $\begin{array}{l}\text { 1. steroid } 5 \alpha \text {-reductase } 2 \\
\text { 2. steroid } 5 \alpha \text { reductase family } \\
\text { 3. androgen and pregnene metabolism } \\
\text { 4. } \mathrm{T} \rightarrow \mathrm{DHT} \text {; } \mathrm{A} 4 \rightarrow \text { androstanedione } \\
\text { 5. restricted (prostate and androgen sensitive tissues) }\end{array}$ \\
\hline 1. SRD5A3 & Chr: $4 \mathrm{q} 12$ & & 1. steroid $5 \alpha$-reductase 3 \\
\hline 2. 79644 & Gene: ...... & aa: 318 & 2. steroid $5 \alpha$ reductase AND polyprenol reductase subfamily \\
\hline 3. $S R D 5 A 3$ & mRNA: 4.1 & Da: 36,521 & 3. androgen and pregnene metabolism \\
\hline $\begin{array}{l}\text { CDG1P; CDG1Q; } \\
\text { KRIZI; SRD5A2L; } \\
\text { SRD5A2L1 }\end{array}$ & Exons: 6 & L: EndRet & $\begin{array}{l}\text { 4. } \mathrm{T} \rightarrow \mathrm{DHT} ; \mathrm{A} 4 \rightarrow \text { androstanedione; } 17 \mathrm{OHP} 4 \rightarrow 5 \alpha \text {-pregnan-17 } \alpha \text {-ol-3,20-dione; } \mathrm{P} \rightarrow 5 \alpha \mathrm{DHP} \text {; } \\
20 \alpha \mathrm{DHP} \rightarrow 5 \alpha \text {-pregnan-20-ol-3-one } \\
\text { 5. ubiquitous }\end{array}$ \\
\hline
\end{tabular}


TABLE 2 | Continued

\begin{tabular}{lllll}
\hline & Chromosome & Protein $(\mathrm{aa})^{\star \star}$ & & \\
1. Abbr. & Gene size* $^{*}$ & Dalton & 1. Name & 2. Family \\
2. Gene ID & mRNA size & Localization (L) $^{\star \star *}$ & 3. Catalysis & 4. Substrates \\
3. Gene name(s) & Exons (no) & & 5. Distribution & 6. Cofactor \\
\hline
\end{tabular}

\begin{tabular}{|c|c|c|c|}
\hline 1. STS & Chr: Xp22.31 & & 1. steroid sulphatase \\
\hline 2. 412 & Gene: 208.3 & aa: 583 & 2. sulphatase \\
\hline 3. STS & mRNA: 6.4 & Dalton: 65,492 & 3. hydrolyses several $3 \beta$-hydroxysteroid sulfates \\
\hline $\begin{array}{l}E S ; \text { ASC; XLI; ARSC; } \\
\text { SSDD; ARSC1 }\end{array}$ & Exons: 16 & L: microsomes EndRet & $\begin{array}{l}\text { 4. sulpho conjugated cholesterol, E1, E2, DHEA, P5, 17OHP5S, A5, EPI } \\
\text { 5. ubiquitous (lung, aorta, thyroid, uterus, liver and testis) }\end{array}$ \\
\hline
\end{tabular}

\begin{tabular}{|c|c|c|c|}
\hline 1. SULT1E1 & Chr: $4 \mathrm{q} 13.3$ & & 1. estrogen sulphotransferase \\
\hline 2. 6783 & Gene: 50.0 & aa: 35126 & 2. sulphotransferase 1 \\
\hline 3. SULT1E1 & mRNA: 1.8 & Dalton: 35,126 & 3. sulpho-conjugation of steroids \\
\hline EST; STE; EST-1; & Exons: 9 & L: cytoplasm & 4. E1, DHEA (low affinity for E2) \\
\hline ST1E1; $\left(\right.$ more $\left.^{\wedge \wedge}\right)$ & & & $\begin{array}{l}\text { 5. moderately distributed (liver, adrenal, small intestine; low in brain, lung, testis, } \\
\text { leukocytes, placenta, salivary gland, stomach, thymus, trachea, uterus, kidney)(30,31) }\end{array}$ \\
\hline
\end{tabular}

\begin{tabular}{|c|c|c|c|}
\hline 1. SULT2A1 & Chr: 19q13.3 & & 1. dehydroepiandrosterone sulphotransferase \\
\hline 2. 6822 & Gene: 15.9 & aa: 285 & 2. sulphotransferase 1 \\
\hline 3. SULT2A1 & mRNA: 2.0 & Dalton: 33,780 & 3. sulpho-conjugation of steroids, bile acids \\
\hline
\end{tabular}

\begin{tabular}{|c|c|c|c|}
\hline 1. SULT2B1 & Chr: $19 q 13.33$ & & 1. alcohol sulphotransferase \\
\hline 2. 6820 & Gene: 48.5 & aa: 365 & 2. sulphotransferase 1 \\
\hline 3. SULT2B1 & mRNA: 1.3 & Dalton: 41,308 & 3. sulpho-conjugation of steroids \\
\hline HSST2; ARCI14 & Exons: 7 & L: cytoplasm & 4. cholesterol, DHEA \\
\hline & & & $\begin{array}{l}\text { 5. moderately distributed (placenta, prostate, lung (low in kidney, salivary gland, small intestine, } \\
\text { trachea) } \text {, }^{30}\end{array}$ \\
\hline
\end{tabular}

\begin{tabular}{|c|c|c|c|}
\hline 1. SULT1A1 & Chr: $16 p 11.2$ & & 1. phenol sulphotransferase 1 \\
\hline 2. 6817 & Gene: 18.4 & aa: 295 & 2. sulphotransferase 1 \\
\hline 3. SULT1A1 & mRNA: 1.3 & Dalton: 34,165 & 3. sulpho-conjugation of steroids \\
\hline $\begin{array}{l}\text { PST; STP; STP1; } \\
\text { P-PST; ST1A1; } \\
\text { ST1A3; TSPST1; } \\
\text { HAST1/HAST2 }\end{array}$ & Exons: 13 & L: cytoplasm & $\begin{array}{l}\text { 4. E2 } \\
\text { 5. nearly ubiquitous (adrenal, bone marrow, brain, colon, hearth, kidney, liver, lung, pancreas, } \\
\text { leukocytes, placenta, prostate, salivary gland, skeletal muscle, small intestine, spinal cord, } \\
\text { spleen, stomach, testis, thymus, thyroid, trachea, uterus) }\end{array}$ \\
\hline
\end{tabular}

List of all enzymes discussed in the present review with the major features. Gene and gene product nomenclature is complex and variable and alias are given as Supplemental Materials.

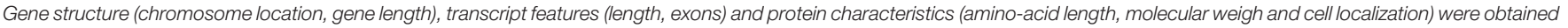
from GeneCards (www.genecards.org. Accessed on date: February 2018) (Stelzer et al., 2016).

* Length in kilo nucleotides.

${ }^{* *}$ number of amino-acids.

*** Abbreviations: EndRet: endoplasmic reticulum; extracell: extracellular; mitochon: mitochondria.

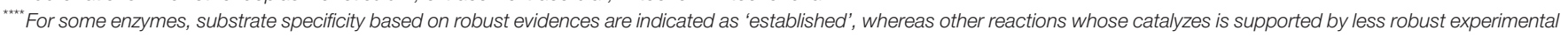
evidences (mostly using recombinant proteins in vitro/cell-free assays) are indicated as "postulated."

\$This reaction of CYP17A1 does not occur in vivo in humans (Miller and Auchus, 2011).

$\$ \$$ \$KR1C4 has an important detoxifying function in the liver and converts chlordecone into chlordecone alcohol.

\&\& $17 \beta H S D 3$ is testis specific and the reactions catalyzed by this enzyme are not reported in Figure 2.

$\wedge \wedge$ For this gene, additional gene names exist, for details see NCBI database (https://www.ncbi.nlm.nih.gov/).

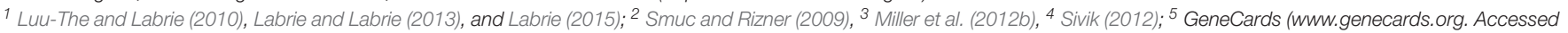

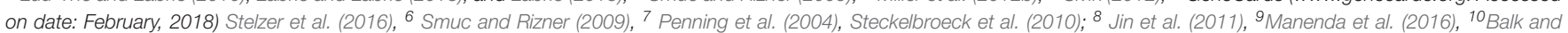

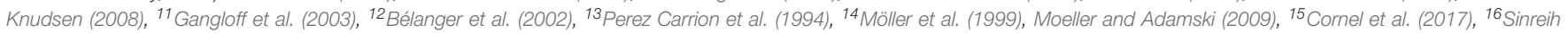

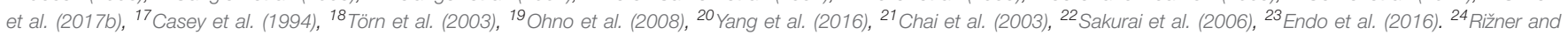

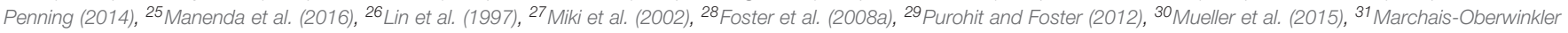
et al. (2011), Mueller et al. (2015), ${ }^{32}$ Rege et al. (2016), ${ }^{33}$ Strott (2002), and ${ }^{34}$ Yang et al. (2016).

By targeting steroid intracrine metabolism, the effects of steroids can be modulated locally. Table 3 overviews the available drugs targeting intracrine enzymes and their developmental status. CYP19A1 (aromatase) inhibitors, currently at their third generation, started to be used for breast cancer during the 80's of last century (Lønning and Eikesdal, 2013), and was followed by drugs able to target other enzymes (CYP11A1, CYP17A1, SRD5As; Table 3). 
More recently, there is a re-emerging interest in developing novel intracrine drugs. A number of compounds are in their clinical phases, like STS inhibitors (Maltais and Poirier, 2011; Woo et al., 2011; Purohit and Foster, 2012; Pohl et al., 2014; Pautier et al., 2017) or inhibitors of AKR1C3/17ßHSD5, which are of particular interest because this enzyme has crucial role in androgen/estrogen and prostaglandin biosynthesis (Penning, 2017). Bayer's AKR1C3/17 $\beta$ HSD5 inhibitor BAY 1128688 has a modified estrogen core, it interferes with both pathways, and is in phase II clinical trial for endometriosis (Bothe et al., 2017). Astellas Pharma potent and selective AKR1C3/17 $\beta$ HSD5 inhibitor ASP-9521 had only modest effect in a phase II study on prostate cancer as single drug, but combination therapy approaches remain to be studied (Kikuchi et al., 2014; Loriot et al., 2014).

HSD inhibitors are being studied in the area of hormonedependent diseases, with 11ßHSD inhibitors being in clinical trials for metabolic disorders (Ye et al., 2017) and 17ßHSD inhibitors approaching the clinical phase for a number of gynecological indications (Table 3; Abdelsamie et al., 2017).

\section{INTRACRINOLOGY IN PERIPHERAL TISSUES}

In this paragraph, intracrinology of endometrium, GIT, bone, lungs, and CNS is reviewed. To comprehensively understand the ability of these tissues and systems to generate estrogens and other steroids, we have performed a systematic search of all original papers published in English until June 2018 that described the levels of intracrine enzymes (those indicated in Table 2-mRNA, protein or activity) in healthy tissues. In total 177 if the four extra ref are allowed papers were reviewed, and for details of this search, see Supplemental panel: "Systematic Review." The results of this systematic review are summarized in Tables 6-8 and are briefly overviewed in each section dedicated to the distinct tissues or systems. Reports describing the enzymes in cultured cells or cell lines were excluded (may have been discussed elsewhere, though). Each section follows then with a non-systematic overview of the role of intracrinology in pathophysiology. A brief non-systematic description of the intracrinology of the skin, immune system and adipose tissue is also given. We will not describe the intracrinology of breast, prostate and liver (where steroid catabolism is the most relevant aspect), and we redirect the reader to recent reviews (Foster et al., 2008a; Luu-The et al., 2008; Luu-The and Labrie, 2010; Labrie and Labrie, 2013; Labrie, 2015; Mueller et al., 2015; Zhao et al., 2016; Hilborn et al., 2017; Penning, 2017).

\section{Endometrium}

The actions of steroid hormones in the endometrium are mediated by hormone-receptors via the classical mechanisms, although non-genomic and rapid signaling are also present (Groothuis et al., 2007; Zwart et al., 2011; Flach and Zwart, 2016; Hewitt et al., 2016). Estrogens and P control the menstrual cycle (Groothuis et al., 2007; Andersen and Ezcurra, 2014) and the endometrium during the window of implantation (WOI), occurring in the mid-luteal phase (Wang and Dey, 2006).

In rats, the WOI is characterized by high E2 plasma levels, and endometrial ER $\alpha$ and PR expression shows specific and varying cytosolic/nuclear patterns (Singh et al., 1996). ER $\alpha$ and PR expression decreases after ovulation and in preimplantation stages in both mice (Vasquez and DeMayo, 2013) and primates (Macaca mulatta) (Ghosh et al., 1999).

Rodent genetic models unraveled some molecular mechanisms underlying the estrogen-dependency of these processes. ER $\alpha-\mathrm{KO}$ mice are infertile, no implantation occurs, endometrium is hypoplastic and estrogen response is absent (Couse and Korach, 1999; Walker and Korach, 2004). Not only its absence, but also sustained estrogen signaling has deleterious effects on endometrial receptivity, as recapitulated by mice with uterine COUP-TFII ablation. These mice exhibit increased estrogen signaling and asynchrony between embryo competency and uterine receptivity with consequent implantation defects. This effect is rescued by treatment with the antiestrogen ICI182780 (Lee et al., 2010). Additionally, the duration of E2 exposure and its dosage affect endometrial receptivity and WOI length in mice (Ma et al., 2003).

Available human data, mostly obtained in the context of assisted reproduction technologies (ART), also indicate that steroid stimulation retards or shortens the luteal phase, the WOI, causes shifts in the appearance of pinopodes (a classical WOI marker) and causes asynchrony between ovarian and menstrual cycles (Devroey et al., 2004).

\section{Intracrinology in Healthy Endometrium-Systematic Search}

Initial studies on steroid hormone metabolism in the endometrium date back to 1965 with first demonstration of the STS activity, followed by investigation on the oxidative and reductive $17 \beta$ HSD activities (Table 6).

Both pre and postmenopausal tissues possess oxidative and reductive $17 \beta \mathrm{HSD}$ activities and the expression of $17 \beta \mathrm{HSD} 1$, $2,4,6,7,8,10,12,14$, and AKR1C3/17ßHSD5 was detected at the mRNA or protein levels. Sulphatase pathway (STS and SULT1E1; recently reviewed by Rižner, 2016), CYP19A1, $3 \beta H S D s$, SRD5As and AKR1Cs are also present, indicating that human endometrium can metabolize sulphated-compounds and DHEA to form androgens and estrogens.

Few $17 \beta$ HSDs have been characterized by IHC. The low expression of $17 \beta \mathrm{HSD} 1$ poses sensitivity problems using standard detection methods (Cornel et al., 2017), and few authors reported endometrial absence of 17 $\beta$ HSD1 (Table 6). Type 1 17ßHSD localizes in the cytoplasm of epithelial cells (Dassen et al., 2007; Colette et al., 2013; Mori et al., 2015; Sinreih et al., 2017a) and it is also detected in primary stroma cells cultured in vitro (Aghajanova et al., 2009; Mori et al., 2015). Type 2 17ßHSD, AKR1C3/17ßHSD5 and $3 \beta \mathrm{HSD} 1$ give strong reactivity in the glandular epithelium (Rhee et al., 2003; Ito et al., 2006; Dassen et al., 2007; Vani et al., 2007; Smuc and Rizner, 2009; Zakharov et al., 2010; Colette et al., 2013; Mori et al., 2015; Sinreih et al., 2017a). 
TABLE 3 | Drugs targeting intracrine enzymes.

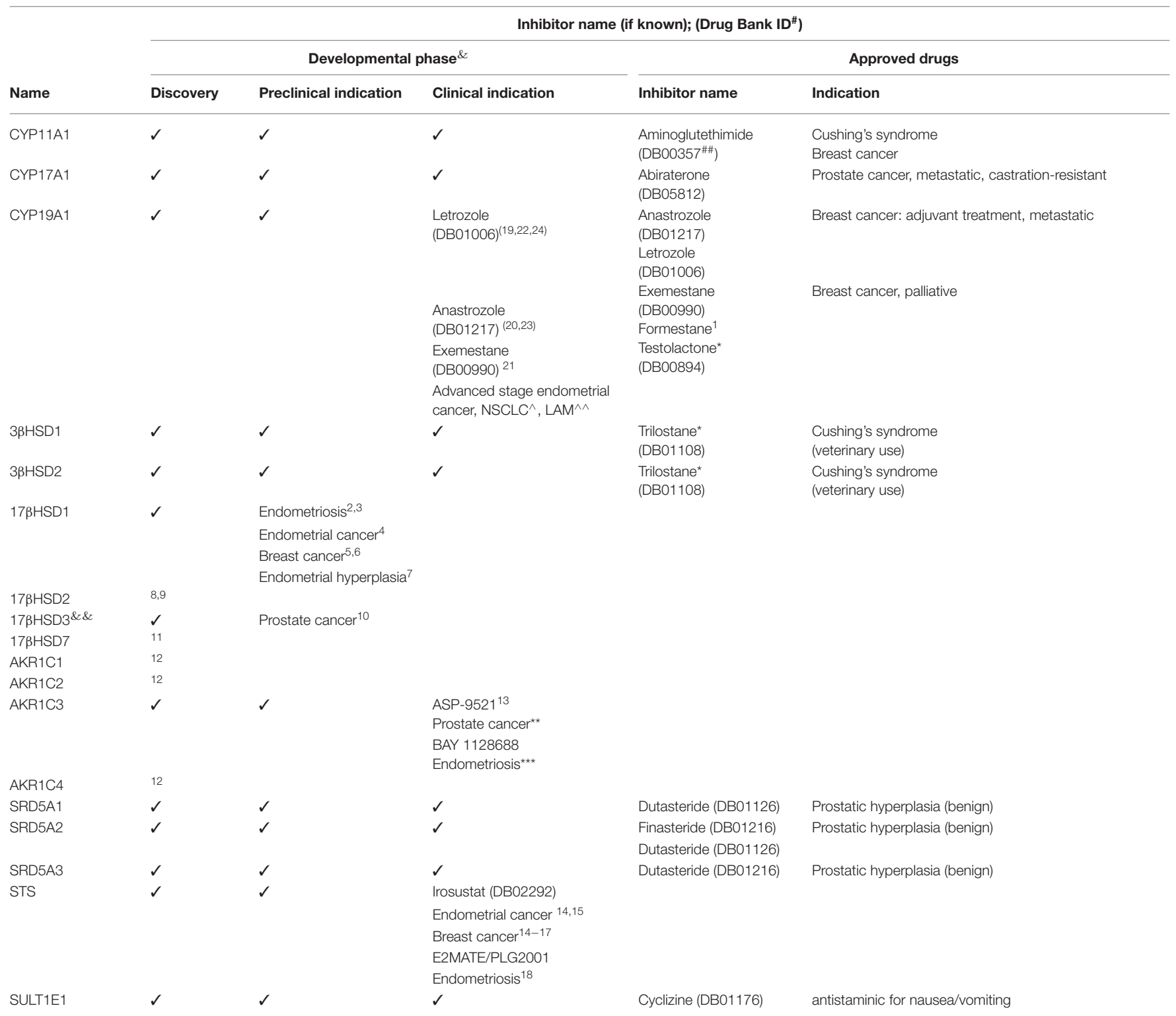

\& 'Clinical phase', i.e., in phase I, II or III trial; 'Preclinical phase' refers to in vivo testing; 'Discovery phase' any previous phase with some candidate compounds.

"Drug Bank ID if the compound is deposited in Drug Bank database (www.drugbank.ca/drugs. Accessed on date: February 2018) (Wishart et al., 2018).

\#\#Aminoglutethimide (ID: DB00357) is an important inhibitor of CYP11A1 with inhibitory activity on CYP19A1 as well.

"No longer on the market.

$\& \& 17 \beta H S D 3$ is testis specific and the reactions catalyzed by this enzyme are not reported in Figure 2.

"This trial for prostate cancer was prematurely terminated (www. clinicaltrials. gov, NCT01352208).

"*t* Phase I trial is concluded (www.clinicaltrials.gov, NCT02434640. Accessed on date: February 2018) and a phase II trial started 2016 (http://adisinsight.springer.com/drugs/ 800041929).

^NSCLC: non-small cell lung cancer

$\wedge \wedge$ LAM: Iymphangioleiomyomatosis

${ }^{1}$ Perez Carrion et al. (1994), ${ }^{2}$ Arnold and Einspanier (2013), ${ }^{3}$ Delvoux et al. (2014), ${ }^{4}$ Konings et al. (2017), ${ }^{5}$ Järvensivu et al. (2018), ${ }^{6}$ Husen et al. (2006), ${ }^{7}$ Saloniemi et al. (2010), ${ }^{8}$ Gargano et al. (2015), ${ }^{9}$ Soubhye et al. (2015) ${ }^{10}$ Day et al. (2013), ${ }^{11}$ Wang et al. (2017), ${ }^{12}$ BroŽic et al. (2011), ${ }^{13}$ Kikuchi et al. (2014), ${ }^{14}$ Purohit and Foster (2012), ${ }^{15}$ Pautier et al. (2017), ${ }^{16}$ Palmieri et al. (2017a), ${ }^{17}$ Palmieri et al. (2017b), ${ }^{18}$ Pohl et al. (2014), ${ }^{19}$ Ma et al. (2004), ${ }^{20}$ Rose et al. (2000), ${ }^{21}$ Lindemann et al. (2014), ${ }^{22}$ Slomovitz et al. (2015), ${ }^{23}$ NCT00932152; ${ }^{25}$ Lu et al. (2017). 
TABLE 4 | Mouse models (knockouts - KO or transgenic-TG, i.e., ubiquitous expression of the gene, unless specified) for intracrine enzymes.

\begin{tabular}{|c|c|c|}
\hline Gene $^{\wedge}$ & $\begin{array}{l}\text { Modification } \\
\text { MGI ID } \$\end{array}$ & Phenotype \\
\hline SatAR & $\begin{array}{l}\text { Null/KO } 1 \\
\text { MGl: } 2388706\end{array}$ & $\begin{array}{l}\text { Endocrine (steroids) \& reproductive endocrinology } \\
\text { - abnormal endocrine organs (adrenal, ovaries, prostate, testis). } \\
\text { - decreased steroids and increased adrenocorticotropin level. } \\
\text { - adrenocortical insufficiency. } \\
\text { - loss of negative feedback regulation at hypothalamic-pituitary levels. } \\
\text { Additional } \\
\text { Growth retardation neo/post natal lethality (incomplete penetrance). Reproductive system: abnormal uterus; } \\
\text { incomplete spermatogenesis; abnormal genitalia. }\end{array}$ \\
\hline \multirow[t]{2}{*}{ CYP11A1 } & $\begin{array}{l}\text { Null/KO² } \\
\text { MGl:5464022 }\end{array}$ & $\begin{array}{l}\text { Endocrine (steroids) and reproductive endocrinology } \\
\text { - abnormal adrenal gland morphology. } \\
\text { - increased circulating adrenocorticotropin level. } \\
\text { - lack of steroid production. } \\
\text { - decreased corticosterone and aldosterone levels. }\end{array}$ \\
\hline & $\begin{array}{l}\text { Null/KO }{ }^{3} \\
\text { MGl: } 2183813\end{array}$ & $\begin{array}{l}\text { Additional } \\
\text { Neonatal lethality (rescued by steroid supplementation); abnormal mitochondrion morphology; abnormal lipid level. } \\
\text { Reproductive system: abnormal genitalia, prostate, testis morphology and spermatogenesis; Nervous system: } \\
\text { abnormal adrenaline and noradrenaline level; abnormal food intake, hypoactivity; postnatal growth retardation. }\end{array}$ \\
\hline \multirow[t]{2}{*}{ CYP17A1 } & $\begin{array}{l}\text { Null/KO }{ }^{4} \\
\text { MGl:3722780 }\end{array}$ & $\begin{array}{l}\text { Endocrine (steroids) \& reproductive endocrinology } \\
\text { - increased circulating cholesterol level. } \\
\text { - decreased T level. } \\
\text { - early reproductive senescence. }\end{array}$ \\
\hline & $\begin{array}{l}\text { Null/KO5 } \\
\text { MGl:3047328 } \\
\text { Null/KO } \\
\text { MGl:5605834 }\end{array}$ & $\begin{array}{l}\text { Additional } \\
\text { Homozygous embryonic lethality (Ed7, between implantation and somite formation). Reproductive system: } \\
\text { abnormal sperm flagellum morphology/asthenozoospermia; reduced male fertility. Bone: abnormal bone } \\
\text { structure, mineral content and density. Metabolism: increased total body fat; decreased lean body mass; } \\
\text { increased circulating creatinine level; increased fasted circulating glucose level. Nervous system: abnormal } \\
\text { sexual interaction. }\end{array}$ \\
\hline \multirow[t]{2}{*}{ CYP19A1 } & $\begin{array}{l}\text { Null/KO } 6 \\
\text { MGl:2179439 }\end{array}$ & $\begin{array}{l}\text { Endocrine (steroids) and reproductive endocrinology } \\
\text { - increased circulating cholesterol, T, DHT, FSH, LH and prolactin. } \\
\text { - decreased circulating E2 level. } \\
\text { - abnormal endometrium (thin, decreased uterus weight). } \\
\text { - abnormal ovary (absence of follicles and corpus luteum, anovulation). }\end{array}$ \\
\hline & $\begin{array}{l}\text { Null/KO } \\
\text { MGl:2154536 } \\
\text { Null/KO } 8 \\
\text { MGl:2389548 }\end{array}$ & $\begin{array}{l}\text { Additional } \\
\text { Reproductive system: ovary hemorrhage and cysts; increased seminal vesicle weight and abnormal } \\
\text { seminiferous tubule epithelium and oligozoospermia; female infertility and reduced male fertility. Metabolism: } \\
\text { increased fat; obesity and susceptibility to weight gain. Bone: decreased bone mineral density and bone mass; } \\
\text { increased bone resorption, osteoclast cell number; abnormal compact and trabecular bone morphology. } \\
\text { Metabolism: increased circulating glucose and triglyceride levels; impaired glucose tolerance; insulin resistance; } \\
\text { hepatic steatosis; abnormal liver physiology. Nervous system: abnormal short term spatial reference memory; } \\
\text { abnormal emotion/affect behavior; abnormal barbering behavior; increased grooming behavior; abnormal } \\
\text { locomotor activation, bradykinesia; abnormal mating frequency. }\end{array}$ \\
\hline
\end{tabular}

\begin{tabular}{ll}
\hline $17 \beta H S D 1$ & Null/KO 9 \\
& MGl:5576042 and \\
& 3799948
\end{tabular}

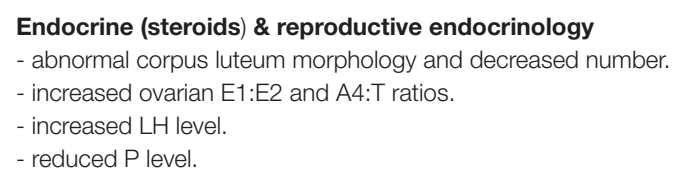
morphology, abnormal retina morphology, abnormal retinal pigmentation. Reproductive system: increased ovary weight; reduced female fertility. Metabolism: decreased circulating glucose level. Nervous system: abnormal behavior, response to light, sleep behavior, decreased exploration in new environment; abnormal motor coordination/balance. 
TABLE 4 | Continued

\begin{tabular}{lll}
\hline Gene $^{\wedge}$ & $\begin{array}{l}\text { Modification } \\
\text { MGI ID }^{\$}\end{array}$ & Phenotype \\
\hline $17 \mathrm{bHSD1}$ & $\mathrm{TG}^{10}$ & \\
& Reproductive endocrinology \\
& - female have increased T levels. \\
& - increased E1 $\rightarrow$ E2 conversion. \\
& - masculinization in females. \\
& - develop benign/malignant breast, ovarian and endometrial conditions.
\end{tabular}

\begin{tabular}{|c|c|c|}
\hline \multirow[t]{2}{*}{ 17ßHSD2 } & $\begin{array}{l}\text { Null/KO11 } \\
\text { MGl:3773836 }\end{array}$ & $\begin{array}{l}\text { No clear reproductive endocrinology phenotype } \\
\text { Additional } \\
\text { Heterozygous mice: growth retardation at birth ant postnatal; premature death; renal degeneration. } \\
\text { Reproductive system: } 70 \% \text { embryonic lethality (Ed11.5) due to placental defects (homozygous); small and } \\
\text { abnormal placenta morphology; Nervous system: brain phenotype with enlarged ventricles; abnormal cortex } \\
\text { morphology; impaired balance, coordination, abnormal sleep pattern, megacephaly. }\end{array}$ \\
\hline & $\mathrm{TG}^{12}$ & $\begin{array}{l}\text { Reproductive endocrinology } \\
\text { - low T level. }\end{array}$ \\
\hline $17 \beta \mathrm{HSD} 4$ & $\mathrm{Null} / \mathrm{KO}^{13}$ & $\begin{array}{l}\text { No clear reproductive endocrinology phenotype } \\
\text { Additional } \\
\text { Neonatal and postnatal lethality; postnatal growth retardation; abnormal mitochondrion morphology; abnormal bile } \\
\text { salt level; hepatic steatosis. Reproductive system: abnormal testis and spermatid morphology; seminiferous } \\
\text { tubule degeneration; small testis; abnormal gametogenesis; reduced male fertility. Nervous system: microgliosis; } \\
\text { Purkinje cell degeneration; astrocytosis; axon degeneration; abnormal suckling behavior; increased anxiety-related } \\
\text { response, tremors, ataxia, impaired coordination, hypoactivity, lethargy; abnormal gait. GIT: abnormal intestinal } \\
\text { absorption. Metabolism: decreased body weight; abnormal lipid homeostasis and decreased fatty acid level. }\end{array}$ \\
\hline
\end{tabular}

\begin{tabular}{|c|c|c|}
\hline \multirow[t]{2}{*}{$17 \beta \mathrm{HSD} 7$} & $\begin{array}{l}\text { Null/KO }{ }^{14} \\
\text { MGl:3811923 }\end{array}$ & $\begin{array}{l}\text { Endocrine (steroids) } \\
\text { Cholesterol biosynthesis. }\end{array}$ \\
\hline & Null/KO ${ }^{15}$ & Additional \\
\hline
\end{tabular}

\begin{tabular}{|c|c|c|}
\hline \multirow[t]{2}{*}{$17 \beta \mathrm{HSD} 9$} & $\begin{array}{l}\text { Null/KO'16 } \\
\text { MGl: } 2446073\end{array}$ & No clear reproductive endocrinology phenotype \\
\hline & $\mathrm{Null} / \mathrm{KO}^{17}$ & Additional \\
\hline \multirow[t]{2}{*}{$17 \beta \mathrm{HSD} 10$} & $\mathrm{Null} / \mathrm{KO}^{18}$ & $\begin{array}{l}\text { No clear reproductive endocrinology phenotype } \\
\text { Additional } \\
\text { Mitochondria dysfunction; reduced plasma glucose and increase insulin levels. Nervous system: neuronal } \\
\text { damage. }\end{array}$ \\
\hline & $\begin{array}{l}\text { TG } \text { (brain } \\
\text { specific }^{19}\end{array}$ & $\begin{array}{l}\text { No clear reproductive endocrinology phenotype } \\
\text { Additional } \\
\text { Nervous system: Protect against ischemia, Parkinson, Alzheimer disease model }\end{array}$ \\
\hline $17 \beta$ HSD11 & $\begin{array}{l}\text { Null/KO20 } \\
\text { MGl:5581418 }\end{array}$ & $\begin{array}{l}\text { No clear reproductive endocrinology phenotype } \\
\text { Additional } \\
\text { Increased total circulating protein level. Nervous system: hyperactivity. }\end{array}$ \\
\hline $17 \beta \mathrm{HSD13}$ & $\begin{array}{l}\text { Null/KO22 } \\
\text { MGl:5007180 }\end{array}$ & No clear phenotype associated. \\
\hline
\end{tabular}


TABLE 4 | Continued

\begin{tabular}{|c|c|c|}
\hline Gene $^{\wedge}$ & $\begin{array}{l}\text { Modification } \\
\text { MGI ID } \$\end{array}$ & Phenotype \\
\hline $17 \beta \mathrm{HSD} 14$ & $\begin{array}{l}\text { Null/KO } 23 \\
\text { MGI:5007181 }\end{array}$ & $\begin{array}{l}\text { No clear reproductive endocrinology phenotype } \\
\text { Additional } \\
\text { Increased IgG2a level. Reproductive system: oligozoospermia, testis degeneration, male infertility. Nervous } \\
\text { system: increased response to stress-induced hyperthermia. }\end{array}$ \\
\hline
\end{tabular}

\begin{tabular}{|c|c|c|}
\hline $17 \beta \mathrm{HSD} 15$ & $\begin{array}{l}\text { Null/KO24 } \\
\text { MGl:3526658 \& } \\
3586379\end{array}$ & $\begin{array}{l}\text { No clear reproductive endocrinology phenotype } \\
\text { Additional } \\
\text { Abnormal eye electrophysiology, delayed dark adaptation }\end{array}$ \\
\hline SRD5A1 & $\begin{array}{l}\mathrm{Null} / \mathrm{KO}^{27} \\
\mathrm{MGl}: 1857454\end{array}$ & $\begin{array}{l}\text { Reproductive endocrinology } \\
\text { - parturition defects, rescued by } 3 \alpha-D I O L \text { supplementation. } \\
\text { Additional } \\
\text { Decreased litter size; small prostate. }\end{array}$ \\
\hline
\end{tabular}

\begin{tabular}{ll}
\hline SRD5A2 & Null/KO28 \\
MGl:2178039 & Reproductive endocrinology \\
& - T accumulation in reproductive tissues. \\
& - impaired androgen-dependent gene expression. \\
& - parturition defects, rescued by $3 \alpha$-DIOL supplementation. \\
& Additional \\
& Decreased litter size; small prostate.
\end{tabular}

\begin{tabular}{|c|c|c|}
\hline SRD5A3 & $\begin{array}{l}\text { Null/KO29 } \\
\text { MGl:5520177 }\end{array}$ & $\begin{array}{l}\text { Mouse not thoroughly characterized } \\
\text { Embryonic lethality, abnormal heart morphology, abnormal neural tube closure }\end{array}$ \\
\hline \multirow[t]{2}{*}{ SULT1E1 } & $\begin{array}{l}\text { Null/KO } 30 \\
\text { MGl:3529586 }\end{array}$ & $\begin{array}{l}\text { Reproductive endocrinology } \\
\text { - elevated circulating estrogen levels. }\end{array}$ \\
\hline & & $\begin{array}{l}\text { Additional } \\
\text { Disturbed platelet physiology. Reproductive system: leyding cell hyperplasia and abnormal morphology; } \\
\text { abnormal testis morphology; abnormal placentation and amniotic fluid composition. }\end{array}$ \\
\hline
\end{tabular}

\begin{tabular}{lll}
\hline SULT2B1 & Null/KO & Endocrine (steroids) \\
& MGl:5432568 & disturbed cholesterol metabolism and levels. \\
& (unpublished) &
\end{tabular}

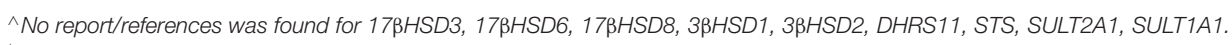

"The human AKR1C3/17BHSD5 KO refers to mice with disrupted AKR1C18, however, functional conservation between the four human AKR1Cs and the eight mouse AKR1Cs in unclear (Sudeshna et al., 2013).

\$Reference ID refers to the Mouse Genome Informatics (MGl; www.informatics.jax.org. Accessed on date: February 2018) (Blake et al., 2017).

${ }^{1}$ Caron et al. (1997), ${ }^{2}$ Huang et al. (2012), ${ }^{3}$ Hu et al. (2002), ${ }^{4}$ Liu et al. (2005), ${ }^{5}$ Bair and Mellon (2004), ${ }^{6}$ Nemoto et al. (2000), ${ }^{7}$ Fisher et al. (1998), ${ }^{8}$ Honda et al. (1998), ${ }^{9}$ Hakkarainen et al. (2015), ${ }^{10}$ Saloniemi et al. (2010) and Järvensivu et al. (2018), ${ }^{11}$ Rantakari et al. (2008), ${ }^{12}$ Zhongyi et al. (2007), ${ }^{13}$ Baes et al. (2000), ${ }^{14}$ Shehu et al. (2008), ${ }^{15}$ Jokela et al. (2010), ${ }^{16}$ Shang et al. (2002), ${ }^{17}$ Driessen et al. (2000) and Sahu et al. (2015); ${ }^{18} \mathrm{Li}$ et al. (2010) and Rauschenberger et al. (2010); ${ }^{19}$ Li et al. (2010); Rauschenberger et al. (2010), 20 Dickinson et al. (2016), ${ }^{21}$ Rantakari et al. (2010); Kemilainen et al. (2016); ${ }^{22}$ Tang et al. (2010), ${ }^{23}$ Tang et al. (2010), ${ }^{24}$ Kim et al. (2005), ${ }^{25}$ Piekorz et al. (2005), ${ }^{26} /$ shida et al. (2007), ${ }^{27}$ Mahendroo et al. (1996), ${ }^{28}$ Mahendroo et al. (2001), ${ }^{29}$ Dickinson et al. (2016), ${ }^{30}$ Qian et al. (2001) and Tong et al. (2005).
}

CYP19A1 as well has low expression and some authors detected this enzyme only in association with diseases (see below and recently reviewed by Rižner, 2013). Although CYP19A1 immunoreactivity was initially associated with stroma cells (Watanabe et al., 1995), subsequent investigations showed also glandular expression (Kitawaki et al., 1999; Hudelist et al., 2007) and laser-capture-microdissected stroma/epithelial components detected CYP19A1 mRNA in both cell types (Matsuzaki et al., 2006).

The mRNA of those enzymes converting cholesterol to DHEA (CYP11A1, CYP17A1, StAR) and (ovarian) $3 \beta \mathrm{HSD} 2$ was reported in recent studies, suggesting that the endometrium can produce steroids from cholesterol (Table 6). 
TABLE 5 | Diseases associated with gene variants in intracrine enzymes.

\begin{tabular}{|c|c|c|c|}
\hline Name & $\begin{array}{l}\text { Affected system or } \\
\text { tissue Disease }\end{array}$ & Phenotype & OMIM ID\# References \\
\hline StAR & $\begin{array}{l}\text { Endocrine system } \\
\text { Lipoid adrenal } \\
\text { hyperplasia }\end{array}$ & $\begin{array}{l}\text { Deficiency of adrenal or gonadal steroids } \\
\text { All individuals are phenotypic females } \\
\text { Infant mortality (mineral- \& glucocorticoid deficiency }\end{array}$ & OMIM: $201710^{1}$ \\
\hline CYP11A1 & $\begin{array}{l}\text { Endocrine system } \\
\text { Congenital adrenal } \\
\text { insufficiency, with } 46 X Y \\
\text { sex reversal } \\
\text { Reproductive }\left(a s s^{\wedge}\right) \\
\text { Bone }\left(a s s^{\wedge}\right) \\
\text { CNV }\left(a s s^{\wedge}\right)\end{array}$ & $\begin{array}{l}\text { Acute adrenal insufficiency in infancy or childhood } \\
\text { Abnormality of cholesterol metabolism } \\
\text { Absence of secondary sex characteristics } \\
\text { SNPs^^ associated with endometrial cancer } \\
\text { SNPs associated with skeletal adverse events to Al } \\
\text { SNPs associated with neurological disturbances }\end{array}$ & OMIM: $613743^{1}$ \\
\hline CYP17A1 & $\begin{array}{l}\text { Endocrine system } \\
\text { Congenital adrenal } \\
\text { insufficiency } \\
(17 \alpha \text {-hydroxyl. } \\
\text { deficiency) } \\
\text { GIT }\left(a s s^{\wedge}\right) \\
\text { Reproductive }\left(a s s^{\wedge}\right)\end{array}$ & $\begin{array}{l}\text { Excessive corticosteroids leading to hypertension } \\
\text { Low aldosterone synthesis } \\
\text { Abnormal sex determination and secondary sex } \\
\text { characteristics, amenorrhea } \\
\text { SNPs associated with risk of cancer in the CRC\#\# } \\
\text { SNPs associated with endometrial cancer }\end{array}$ & OMIM: $202110^{1}$ \\
\hline \multirow[t]{5}{*}{ CYP19A1 } & $\begin{array}{l}\text { Reprod Endocrin }{ }^{\star *} \\
\text { Aromatase deficiency }\end{array}$ & $\begin{array}{l}\text { Pseudo hermaphroditism in female } \\
\text { Cystic ovaries, delayed bone maturation, adiposity }\end{array}$ & OMIM: 613546 \\
\hline & $\begin{array}{l}\text { Aromatase excess } \\
\text { syndrome }\end{array}$ & $\begin{array}{l}\text { Heterosexual precocity and gynecomastia in males } \\
\text { Isosexual precocity in females }\end{array}$ & OMIM: 139300 \\
\hline & Reproductive $\left(a s s^{\wedge}\right)$ & $\begin{array}{l}\text { SNPs associated with endometrial cancer and ovarian cancer risks, endometriosis risk and risk } \\
\text { to develop preeclampsia }\end{array}$ & $6-14$ \\
\hline & Bone $\left(a s s^{\wedge}\right)$ & SNPs associated with osteoporosis and fracture risk & 15,16 \\
\hline & Lung $\left(a s s^{\wedge}\right)$ & SNPs associated with lung cancer & 17 \\
\hline & Urogenital $\left(a s s^{\wedge}\right)$ & SNPs associated with bladder cancer & 21 \\
\hline $17 \beta \mathrm{HSD} 1$ & Reproductive $\left(a s s^{\wedge}\right)$ & SNPs associated with E2 levels and with endometriosis, cancer risk, abortion & $22-25$ \\
\hline $17 \beta \mathrm{HSD} 3$ & $\begin{array}{l}\text { Reprod Endocrin } \\
\text { ketosteroidreductase } \\
\text { deficiency of testis }\end{array}$ & $\begin{array}{l}\text { Males: pseudo hermaphroditism, gynecomastia } \\
\text { Infertility }\end{array}$ & OMIM: $264300^{1}$ \\
\hline \multirow[t]{2}{*}{ 17קHSD4 } & $\begin{array}{l}\text { CNS and } \\
\text { Reprod Endocrin } \\
\text { Perrault syndrome } 1\end{array}$ & $\begin{array}{l}\text { Ovarian dysgenesis, amenorrhea, low estrogens } \\
\text { Sensorineural deafness, } \\
\text { Neurologic manifestations (mild mental retardation) }\end{array}$ & OMIM: 233400 \\
\hline & $\begin{array}{l}\text { CNS } \\
\text { D-bifunctional protein } \\
\text { deficiency }\end{array}$ & $\begin{array}{l}\text { Abnormal peroxisomal fatty acid beta-oxidation } \\
\text { Deterioration of nervous system functions } \\
\text { Infantile-onset of hypotonia, seizures, and abnormal facial features, death before the age of } 2 \\
\text { years }\end{array}$ & OMIM: 261515 \\
\hline
\end{tabular}


TABLE 5 | Continued

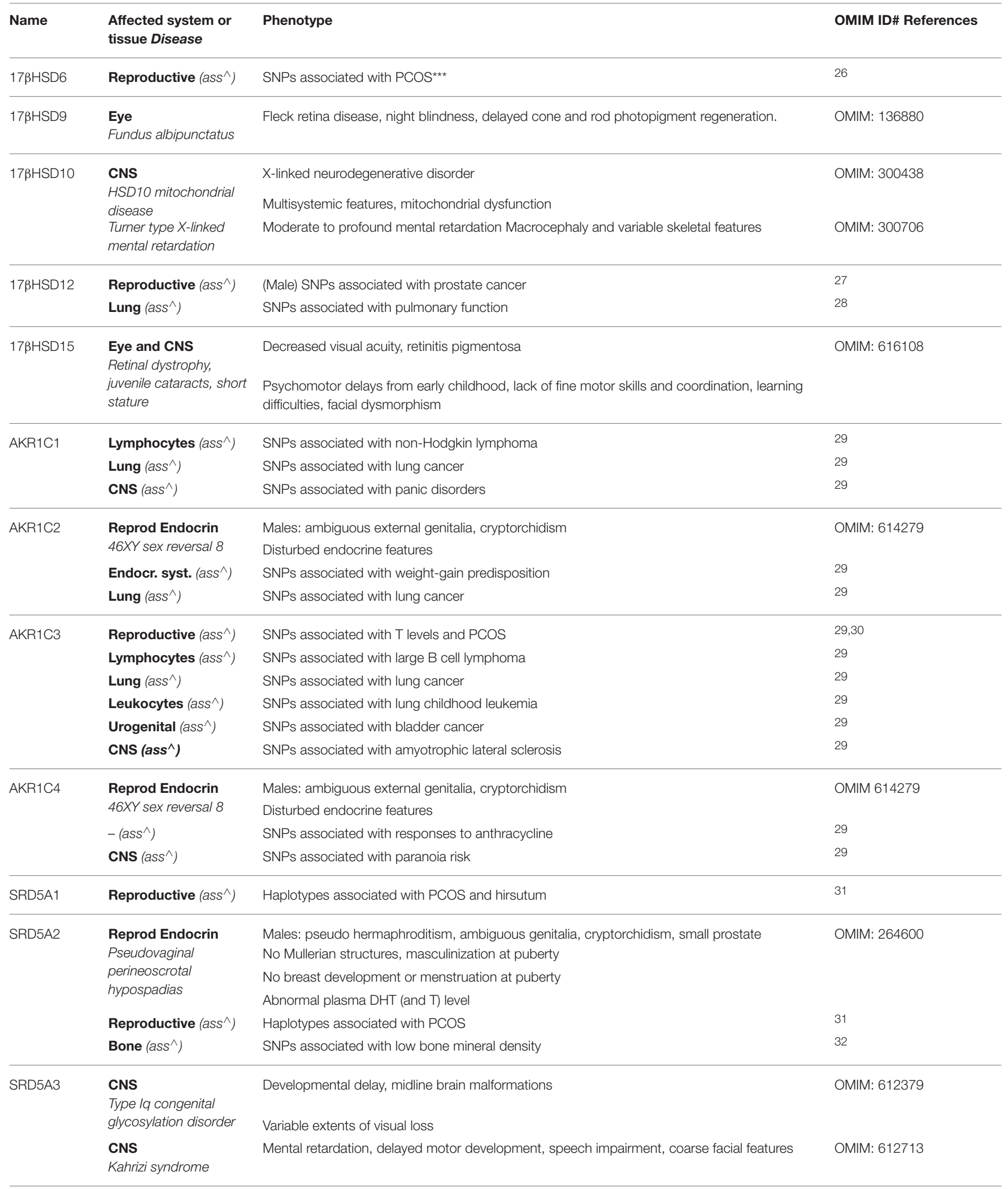


TABLE 5 | Continued

\begin{tabular}{|c|c|c|c|}
\hline Name & $\begin{array}{l}\text { Affected system or } \\
\text { tissue Disease }\end{array}$ & Phenotype & OMIM ID\# References \\
\hline \multirow[t]{2}{*}{ STS } & $\begin{array}{l}\text { Skin } \\
\text { X-linked ichthyosis }\end{array}$ & Cutaneous manifestations: dark brown, polygonal scales and generalized dryness & OMIM: $308100^{40}$ \\
\hline & $\begin{array}{l}\text { Bone } \\
\text { bone dysplasia }\end{array}$ & Chondrodysplasia punctata and bone dysplasia & 33 \\
\hline SULT1E1 & Reproductive $\left(a s s^{\wedge}\right)$ & SNP associated with estrogen dependent diseases & 34 \\
\hline SULT2A1 & Reproductive $\left(a s s^{\wedge}\right)$ & SNP associated with DHEA-S, androgens and PCOS & 40,35 \\
\hline SULT2B1 & $\begin{array}{l}\text { Skin } \\
\text { congenital autosomal } \\
\text { recessive ichthyosis }\end{array}$ & Generalized desquamation, dry scaly skin, hyperkeratosis, erythema & OMIM: 604125 \\
\hline \multirow[t]{4}{*}{ SULT1A1 } & Reproductive $\left(a s s^{\wedge}\right)$ & SNPs associated with endometrial cancer & 11,36 \\
\hline & Bone $\left(a s s^{\wedge}\right)$ & SNPs associated with low bone mineral density & 32 \\
\hline & $\mathbf{G I T}\left(a s s^{\wedge}\right)$ & SNPs associated with risk of cancer in the GIT & $37-38$ \\
\hline & $-\left(a s s^{\wedge}\right)$ & SNPs associated with activity and termostability & 37,39 \\
\hline
\end{tabular}

Selected papers reporting association between SNPs and diseases are reported. Association studies with enzymes involved in steroid signaling but not discusses in the present review exist (for some references, see Doherty et al., 2005; Freedman et al., 2009; Miller and Auchus, 2011; Mueller et al., 2015).

"Endocr syst: Endocrine system.

${ }^{* *}$ Reprod Endocrin: Reproductive endocrinology.

${ }^{* * *}$ PCOS: polycystic ovarian syndrome.

^ass: association studies, case controls.

^^SNP: single nucleotide polymorphism.

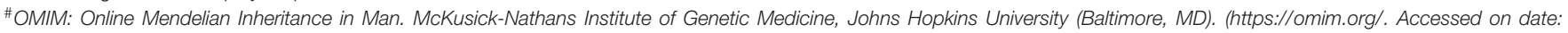
February 2018).

\#\#CRC: colorectal cancer.

${ }^{1}$ Miller and Auchus (2011), ${ }^{2}$ Terry et al. (2010), ${ }^{3}$ Rodríguez-Sanz et al. (2015), ${ }^{4}$ Deng et al. (2016), ${ }^{5}$ Zeng et al. (2016), ${ }^{6}$ Olson et al. (2007), ${ }^{7}$ Berstein et al. (2006), ${ }^{8}$ Kitawaki et al. (2002), ${ }^{9}$ Lundin et al. (2012), ${ }^{10}$ Thompson et al. (2016), ${ }^{11}$ Gulyaeva et al. (2008), ${ }^{12}$ Setiawan et al. (2009), ${ }^{13}$ Zacher et al. (2016), ${ }^{14}$ Shimodaira et al. (2012), 15 Fontein et al. (2014), ${ }^{16}$ Masi et al. (2001), ${ }^{17}$ Zhang et al. (2013), ${ }^{18}$ Cho et al. (2012), ${ }^{19}$ Shimodaira et al. (2010), ${ }^{20}$ Yang et al. (2013), ${ }^{21}$ Andrew et al. (2012), ${ }^{22}$ Tsuchiya et al. (2005), 23 Huber et al. (2005), ${ }^{24}$ Setiawan et al. (2004), ${ }^{25}$ Shi et al. (2016), ${ }^{26}$ Jones et al. (2009), ${ }^{27}$ Audet-Walsh et al. (2012), ${ }^{28}$ Loth et al. (2014), ${ }^{29}$ Alshogran (2017), ${ }^{30}$ Qin et al. (2006), ${ }^{31}$ Goodarzi et al. (2006),

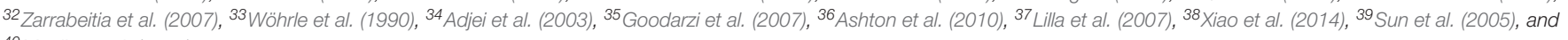
${ }^{40}$ Mueller et al. (2015).

Bold text indicates the system affected, italics text indicates the name of the disease.

\section{Intratissue Steroid Levels}

Endometrial steroid levels were recently profiled by LC-MS. E2 levels differ between tissue and serum during the menstrual cycle, being up to five-times higher in tissue than in serum during the proliferative phase and 1.5-fold higher in the luteal period (Huhtinen et al., 2012a, 2014). T levels were lower in tissue than in serum with no cyclic changes. The levels P and P5 (and their 17-hydroxy derivatives) did not vary between serum and tissue, indicating that, contrarily to estrogens, progestogen intratissue levels are determined by passive diffusion from the blood (Huhtinen et al., 2014).

\section{Intracrinology and Reproduction}

Animal models show not only that intracrine enzymes are expressed in the endometrium, but also they vary the expression levels during the endometrial phases and during implantation, as shown already during the 80's in rhesus monkeys for the oxidizing 17 $\beta$ HSD activity (Kreitmann et al., 1979).

In rodents, STS activity measured with [3H]E1-S in 6-days pregnant rats was lower around the implantation site compared with non-implantation sites (Loza, 1995). In situ hybridisation signal of $17 \beta \mathrm{HSD} 7 \mathrm{mRNA}$ varied spatio-temporally throughout implantation and early gestation, being initially detected on luminal epithelium around the implantation site and absent in decidua (embryonic day, Ed5.5). At Ed8 and Ed9.5, $17 \beta$ HSD7 expression increased in the decidua capsularis (the part that interacts with the trophoblast) and later (after E9) in the junctional zone of the developing placenta and in the spongiotrophoblasts (Nokelainen et al., 2000).

A brilliant study in mice showed that decidualization is dependent on local E2 produced through CYP19A1. CYP19A1 expression increased during pregnancy and decidualization was unaffected by ovariectomy. In contrast, treatment with the aromatase inhibitor (AI) letrozole impaired decidualization and decreased decidual marker expression (e.g., PRP, BMP2 and CX43) (Das et al., 2009).

In human endometrium, 17ßHSD2 and SULT1E1 are induced by $\mathrm{P}$ as their expression peaks in the luteal phase (Rubin et al., 1999; Tseng and Mazella, 2002; Utsunomiya et al., 2004; Dassen et al., 2007; Huhtinen et al., 2012a; Colette et al., 2013; Piccinato et al., 2016b). Since both enzymes decrease intra-tissue estrogen levels, their up-regulation is one of the mechanisms of the uterine antiestrogenic effects of P. The P-dependency of 17 $\beta$ HSD2 and SULT1E1 was recapitulated in vitro using explant cultures and 
TABLE 6 | Expression of intracrine enzymes in endometrium-results of the systematic search.

\begin{tabular}{|c|c|c|c|c|c|}
\hline \multirow[t]{2}{*}{ Name } & & \multicolumn{4}{|c|}{ Menopausal status } \\
\hline & & Technique $\#$ & Pre & Post & References \\
\hline StAR & mRNA & RT-PCR & yes & yes & Bukulmez et al., 2008a; Attar et al., 2009; Sinreih et al., 2017b\& \\
\hline \multirow[t]{2}{*}{ CYP11A1 } & mRNA & RT-PCR & yes & n.d. & Tsai et al., 2001; Rhee et al., 2003; Attar et al., 2009; Sinreih et al., 2013; Huhtinen et al., 2014 \\
\hline & & RT-PCR & no & n.d. & Rhee et al., 2003 \\
\hline \multirow[t]{3}{*}{ CYP17A1 } & mRNA & RT-PCR & yes & n.d. & Tsai et al., 2001; Attar et al., 2009; Huhtinen et al., 2014 \\
\hline & & RT-PCR & no & n.d. & Rhee et al., 2003 \\
\hline & & $\boxplus \mathrm{HC}$ & no & no & Watanabe et al., 1995 \\
\hline \multirow[t]{8}{*}{ CYP19A1 } & mRNA & RT-PCR & yes & yes & $\begin{array}{l}\text { Dheenadayalu et al., 2002; Brosens et al., 2004; Matsuzaki et al., 2006; Pathirage et al., 2006; } \\
\text { Smuc et al., 2006, 2009; Dassen et al., 2007; Bukulmez et al., 2008b; Attar et al., 2009; Smuc } \\
\text { and Rizner, 2009; Lépine et al., 2010; Cornel et al., 2012; Huhtinen et al., 2012a; Delvoux } \\
\text { et al., 2014; Sinreih et al., 2017a }\end{array}$ \\
\hline & & RT-PCR & no & no & $\begin{array}{l}\text { Bulun et al., 1993, 1994; Watanabe et al., 1995; Noble et al., 1996, 1997; Kitawaki et al., } \\
\text { 1999; Bacallao et al., 2008; Colette et al., } 2009\end{array}$ \\
\hline & & ISH & no & n.d. & Watanabe et al., 1995 \\
\hline & Protein & IHC & yes & yes & $\begin{array}{l}\text { Maentausta et al., 1990; Kitaoka et al., 2004; Maia et al., 2006, 2007; Hudelist et al., 2007; } \\
\text { Vouk et al., 2011; Miller et al., 2012a }\end{array}$ \\
\hline & & $\mathrm{IHC}$ & no & no & $\begin{array}{l}\text { Watanabe et al., 1995; Kitawaki et al., 1999; Velasco et al., 2006; Acién et al., 2007; Jeon } \\
\text { et al., 2007; Bukulmez et al., 2008b; Colette et al., } 2009\end{array}$ \\
\hline & & WB & N.d. & yes & Knapp et al., 2013 \\
\hline & & activity & no & no & Bulun et al., 1993; Watanabe et al., 1995; Noble et al., 1997 \\
\hline & & activity & yes & yes & $\begin{array}{l}\text { Tseng et al., 1982; Yamaki et al., 1985; Taga et al., 1990; Yamamoto et al., 1990a,b, 1993a,b; } \\
\text { Jongen et al., 2005; Purohit et al., } 2008\end{array}$ \\
\hline \multirow[t]{3}{*}{ 3ßHSD1 } & mRNA & RT-PCR & yes & yes & $\begin{array}{l}\text { Rhee et al., 2003; Vani et al., 2007; Attar et al., 2009; Smuc et al., 2009; Gibson et al., 2013; } \\
\text { Sinreih et al., } 2013\end{array}$ \\
\hline & Protein $^{\star}$ & IHC & yes & n.d. & Rhee et al., 2003; Vani et al., 2007 \\
\hline & & IHC & no & no & Watanabe et al., 1995 \\
\hline 3ßHSD2 & mRNA & RT-PCR & yes & yes & Tsai et al., 2001; Attar et al., 2009; Huhtinen et al., 2014; Osinski et al., 2018 \\
\hline \multicolumn{6}{|l|}{$17 \beta$ HSDs } \\
\hline $\begin{array}{l}\text { oxidative } \\
\text { activity }\end{array}$ & & & yes & yes & $\begin{array}{l}\text { Tseng and Gurpide, 1974; Pollow et al., 1975a,b, 1976; Polow et al., 1975; Tseng et al., 1977; } \\
\text { Lane, 1990; Kitawaki et al., 2000; Utsunomiya et al., 2001; Delvoux et al., 2007, 2009; Cornel } \\
\text { et al., } 2012\end{array}$ \\
\hline \multirow{2}{*}{$\begin{array}{l}\text { reductive } \\
\text { activity }\end{array}$} & & & yes & yes & Maentausta et al., 1990; Delvoux et al., 2007, 2009, 2014; Bacallao et al., 2008 \\
\hline & & & no & no & Utsunomiya et al., 2001 \\
\hline \multirow[t]{5}{*}{$17 \beta \mathrm{HSD} 1$} & mRNA & RT-PCR & yes & yes & $\begin{array}{l}\text { Zeitoun et al., 1998; Dassen et al., 2007; Smuc et al., 2007, 2009; Bacallao et al., 2008; } \\
\text { Huhtinen et al., 2012a; Colette et al., 2013; Delvoux et al., 2014; Sinreih et al., 2017a; Osinski } \\
\text { et al., } 2018\end{array}$ \\
\hline & & NB & yes & n.d. & Zeitoun et al., 1998 \\
\hline & & RT-PCR & no & no & Casey et al., 1994; Utsunomiya et al., 2001 \\
\hline & Protein & IHC & yes & yes & $\begin{array}{l}\text { Maentausta et al., 1990\&; Mäentausta et al., 1991; Li et al., 2003; Dassen et al., 2007; Colette } \\
\text { et al., 2013; Mori et al., 2015; He et al., 2016; Sinreih et al., 2017a }\end{array}$ \\
\hline & & $\mathrm{HC}$ & no & no & Utsunomiya et al., 2001 \\
\hline \multirow[t]{3}{*}{ 17ßHSD2 } & mRNA & RT-PCR & yes & yes & $\begin{array}{l}\text { Mäentausta et al., 1991; Zeitoun et al., 1998; Kitawaki et al., 2000, 2002; Utsunomiya et al., } \\
\text { 2001; Matsuzaki et al., 2006; Smuc et al., 2006, 2007, 2009; Carneiro et al., 2007; Dassen } \\
\text { et al., 2007; Vani et al., 2007; Bacallao et al., 2008; Hevir et al., 2011b; Huhtinen et al., 2012a; } \\
\text { Colette et al., 2013; Delvoux et al., 2014; Sinreih et al., 2017a; Osinski et al., } 2018\end{array}$ \\
\hline & & NB & yes & n.d. & Zeitoun et al., 1998 \\
\hline & Protein & $\Vdash \mathrm{HC}$ & yes & yes & $\begin{array}{l}\text { Scublinsky et al., 1976; Ciuffi et al., 1982; Utsunomiya et al., 2001; Dassen et al., 2007; Colette } \\
\text { et al., 2013; Cornel et al., 2017; Sinreih et al., 2017a }\end{array}$ \\
\hline \multirow[t]{2}{*}{$17 \beta \mathrm{HSD} 4$} & mRNA & RT-PCR & yes & yes & Dassen et al., 2007; Smuc et al., 2009; Huhtinen et al., 2012a; Delvoux et al., 2014 \\
\hline & & NB & yes & n.d. & Möller et al., 1999 \\
\hline $17 \beta$ HSD6 & mRNA & RT-PCR & yes & n.d. & Huang and Luu-The, 2000; Huhtinen et al., 2012a \\
\hline $17 \beta \mathrm{HSD7}$ & mRNA & RT-PCR & yes & yes & $\begin{array}{l}\text { Smuc et al., 2007, 2009; Smuc and Rizner, 2009; Lépine et al., 2010; Cornel et al., 2012; } \\
\text { Huhtinen et al., 2012a; Delvoux et al., } 2014\end{array}$ \\
\hline
\end{tabular}


TABLE 6 | Continued

\begin{tabular}{|c|c|c|c|c|c|}
\hline \multirow[t]{2}{*}{ Name } & & \multicolumn{4}{|c|}{ Menopausal status } \\
\hline & & Technique $^{\#}$ & Pre & Post & References \\
\hline $17 \beta \mathrm{HSD} 8$ & mRNA & RT-PCR & yes & yes & Smuc and Rizner, 2009; Smuc et al., 2009 \\
\hline $17 \beta \mathrm{HSD} 10$ & mRNA & RT-PCR & yes & n.d. & Huhtinen et al., $2012 a$ \\
\hline $17 \beta \mathrm{HSD} 12$ & mRNA & RT-PCR & yes & yes & $\begin{array}{l}\text { Smuc and Rizner, 2009; Smuc et al., 2009; Lépine et al., 2010; Cornel et al., 2012; Huhtinen } \\
\text { et al., 2012a; Delvoux et al., } 2014\end{array}$ \\
\hline $17 \beta \mathrm{HSD} 14$ & mRNA & RT-PCR & yes & n.d. & Huhtinen et al., 2012a; Sinreih et al., 2017a \\
\hline \multicolumn{6}{|l|}{ AKR1Cs } \\
\hline AKR1C1 & mRNA & RT-PCR & yes & yes & $\begin{array}{l}\text { Rizner et al., 2006; Smuc and Rizner, 2009; Smuc et al., 2009; Hevir et al., 2011b; Sinreih } \\
\text { et al., } 2013\end{array}$ \\
\hline AKR1C2 & mRNA & RT-PCR & yes & yes & Hevir et al., 2011b; Sinreih et al., 2013 \\
\hline \multirow[t]{2}{*}{$\begin{array}{l}\text { AKR1C3/17 } \\
\beta \mathrm{HSD5}\end{array}$} & mRNA & RT-PCR & yes & yes & $\begin{array}{l}\text { Penning et al., 2000; Rizner et al., 2006; Vani et al., 2007; Smuc and Rizner, 2009; Smuc et al., } \\
\text { 2009; Hevir et al., 2011b; Cornel et al., 2012; Huhtinen et al., 2012a; Sinreih et al., 2013; } \\
\text { Delvoux et al., } 2014\end{array}$ \\
\hline & Protein & IHC & yes & yes & $\begin{array}{l}\text { Pelletier et al., 1999; Ito et al., 2006; Vani et al., 2007; Smuc and Rizner, 2009; Zakharov et al., } \\
2010\end{array}$ \\
\hline \multicolumn{6}{|l|}{ SRD5As } \\
\hline \multirow[t]{2}{*}{ SRD5A1 } & mRNA & RT-PCR & yes & yes & Carneiro et al., 2008; Hevir et al., 2011b; Sinreih et al., 2013; Huhtinen et al., 2014 \\
\hline & Protein & $\mathrm{IHC}$ & yes & yes & Ito et al., 2002; Carneiro et al., 2008; Tanaka et al., 2015 \\
\hline \multirow[t]{2}{*}{ SRD5A2 } & mRNA & RT-PCR & yes & yes & Carneiro et al., 2008; Hevir et al., 2011b; Sinreih et al., 2013; Huhtinen et al., 2014 \\
\hline & Protein & $\mathrm{IHC}$ & yes & yes & Ito et al., 2002; Carneiro et al., 2008; Tanaka et al., 2015 \\
\hline SRD5A3 & & RT-PCR & yes & n.d. & Huhtinen et al., 2014 \\
\hline \multicolumn{6}{|l|}{$\begin{array}{l}\text { Sulphatase } \\
\text { pathway }\end{array}$} \\
\hline \multirow[t]{7}{*}{ STS } & mRNA & RT-PCR & yes & yes & $\begin{array}{l}\text { Tanaka et al., 2003; Utsunomiya et al., 2004; Smuc et al., 2006, 2007, 2009; Dalla Valle et al., } \\
\text { 2007; Dassen et al., 2007; Bacallao et al., 2008; Smuc and Rizner, 2009; Lépine et al., 2010; } \\
\text { Colette et al., 2013; Huhtinen et al., 2014; Piccinato et al., 2016b; Sinreih et al., 2017a }\end{array}$ \\
\hline & & RT-PCR & no & no & Miki et al., 2002 \\
\hline & & Comp-RT & yes & n.d. & Yanaihara et al., 2001 \\
\hline & Protein & $\mathrm{IHC}$ & yes & yes & $\begin{array}{l}\text { Yanaihara et al., 2001; Utsunomiya et al., 2004; Dassen et al., 2007; Cornel et al., 2017; } \\
\text { Sinreih et al., 2017a }\end{array}$ \\
\hline & & $\mathrm{HC}$ & no & no & Miki et al., 2002 \\
\hline & & activity & yes & yes & $\begin{array}{l}\text { Warren and French, 1965; Prost and Adessi, 1983; Adessi et al., 1984; Platia et al., 1984; } \\
\text { Yamamoto et al., 1990a, 1993a; Tanaka et al., 2003; Bacallao et al., 2008; Purohit et al., 2008; } \\
\text { Delvoux et al., } 2009\end{array}$ \\
\hline & & $\mathrm{IHC}$ & no & no & Utsunomiya et al., 2004 \\
\hline \multirow[t]{4}{*}{ SULT1E1 } & mRNA & RT-PCR & yes & yes & $\begin{array}{l}\text { Yamamoto et al., 1993a; Miki et al., 2002; Tanaka et al., 2003; Utsunomiya et al., 2004; Smuc } \\
\text { et al., 2006, 2007; Dassen et al., 2007; Bacallao et al., 2008; Smuc and Rizner, 2009; Lépine } \\
\text { et al., 2010; Hevir et al., 2011a, 2013; Colette et al., 2013; Piccinato et al., 2016b; Sinreih } \\
\text { et al., 2017a }\end{array}$ \\
\hline & & NB & yes & n.d. & Rubin et al., 1999 \\
\hline & Protein & $\mathrm{HC}$ & yes & yes & $\begin{array}{l}\text { Miki et al., 2002; Utsunomiya et al., 2004; Hudelist et al., 2007; Cornel et al., 2017; Sinreih } \\
\text { et al., 2017a }\end{array}$ \\
\hline & & activity & yes & yes & Tanaka et al., 2003; Utsunomiya et al., 2004; Bacallao et al., 2008; Purohit et al., 2008 \\
\hline SULT1A1 & mRNA & RT-PCR & yes & yes & Hevir et al., 2011a, 2013 \\
\hline SULT1A1 & & NB & yes & n.d. & Rubin et al., 1999*** \\
\hline SULT2A1 & & NB & no & n.d. & Rubin et al., 1999 \\
\hline SULT2B1 & mRNA & RT-PCR & yes & yes & Hevir et al., 2011a, 2013 \\
\hline
\end{tabular}

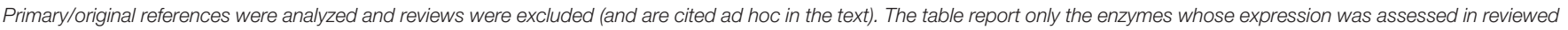
studies ${ }^{* *}$.

\#Technique abbreviations. For mRNA detection, NB: northern blot; ISH: in situ hybridisation; RT-PCR: reverse transcription semi or quantitative PCR; Comp-RT: competitive RT-PCR. For protein detection: IHC: immunohistochemistry; WB: western blotting. For enzyme activity measurement: activity.

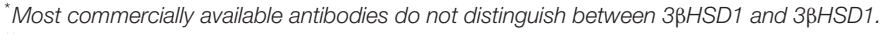

${ }^{* *}$ No publication was found describing the expression of 17BHSD9, 11, 13, 15, DHRS11 and AKR1C4.

${ }^{* * *}$ The same study also detected expression of SULT1A3 (Rubin et al., 1999).

n.d.: not determined.

\& Protein level was measured by radioimmunoassay. 
primary cells (Tseng and Mazella, 2002; Dassen et al., 2007; Piccinato et al., 2016b). Luteal peak expression of other SULTs (1A1 and 2B1) was also reported (Rubin et al., 1999; Koizumi et al., 2010). Some reports also suggested that STS expression increased in the luteal phase (Tanaka et al., 2003; Piccinato et al., 2016b) with a potential role during decidualization (Tseng and Mazella, 2002). Mid-luteal phase endometrium shows also peaking expression of $3 \beta \mathrm{HSD} 1$ (mRNA and protein) (Rhee et al., 2003; Vani et al., 2007).

Two studies on human ectopic pregnancies explored the endometrium around the implanted blastocyst. Expression of $3 \beta \mathrm{HSD} 1$ (mRNA and protein) was highest in decidua obtained from ectopic pregnancies (Rhee et al., 2003) and in a study on 23 tubal pregnancies, $17 \beta$ HSD1 showed highest immunoreactivity at the fetal-maternal interface (Li et al., 2003), suggestive for a role of these enzymes in the nidation site.

\section{Endometriosis}

Endometriosis, an estrogen-dependent benign disorder affecting up to $10 \%$ of reproductive-aged women, is associated with pelvic pain, infertility, decreased life-quality and important health care/social costs (Simoens et al., 2011, 2012; De Graaff et al., 2013, 2015, 2016; Vercellini et al., 2014). Endometriosis is characterized by the growth of endometrium-like tissue outside the uterus (ectopic locations), beside the ovaries (endometrioma), as peritoneal implants, or as deep-lesions infiltrating peritoneal organs (deep endometriosis).

The expression of intracrine enzymes in endometriosis was reviewed in 2012, (Huhtinen et al., 2012b) and among other studies, 20 papers published between 1996 and 2009 specifically described the levels of intracrine enzymes in eutopic and ectopic endometrium from patients and control women. With the exclusion of one study that included over 100 patients (Colette et al., 2009), the rest included small study populations, and in most cases, the various endometriosis types (ovarian, peritoneal and deep infiltrating) were pooled together. Various techniques were used (RT-qPCR, immunohistochemistry, enzyme activity assay). Overall, no clear conclusion could be drawn from these studies. Comparing endometriosis with controls, CYP19A1 was up-regulated (six studies), unchanged (three studies) and one study found no expression of this gene. With respect to oxidative and reductive $17 \beta$ HSDs, $17 \beta$ HSD1 was reported upregulated (three studies), $17 \beta \mathrm{HSD} 2$ was reported down-regulated or unchanged and two studies reported an up-regulation of $17 \beta$ HSD7 and 12 in endometriosis vs. controls (Huhtinen et al., 2012b).

Subsequent investigations also continued to report inconsistent results. No change in mRNA (Delvoux et al., 2014) or increased expression of CYP19A1 in ovarian endometriosis vs. controls (Huhtinen et al., 2012a) were reported. An increased expression of CYP19A1 was also described using in vitro spheroids derived from endometrial stroma cells from patients compared with controls (Mori et al., 2015).

The mRNA expression of $17 \beta$ HSD1 was higher in endometriosis compared with normal tissue using patient biopsies as well as spheroid cultures derived from endometrial stroma cells of patients and controls (Delvoux et al., 2014; Mori et al., 2015). One study assessing the three endometriosis types separately (60 patients in total) described that the increased $17 \beta$ HSD1 level was restricted to endometrioma during the secretory phase of the menstrual cycle (Huhtinen et al., 2012a), whereas a second study on 79 patients and 41 controls, found no change in $17 \beta$ HSD 1 level, but described an increased 17 $\beta$ HSD1/2 ratio (Colette et al., 2013).

Regarding $17 \beta \mathrm{HSD} 2$, recent investigations reported both unchanged (Delvoux et al., 2014) and down-regulated mRNA in patient biopsies compared with controls (Huhtinen et al., 2012a; Colette et al., 2013). No variations were found in 17ßHSD4, 5, 7 and 12 (Smuc et al., 2009; Delvoux et al., 2014) but an increased level of $17 \beta$ HSD6 mRNA was detected in endometriosis compared with controls (Huhtinen et al., 2012a).

A few studies reported detectable levels of the enzymes involved in the generation of DHEA from cholesterol (StAR, CYP11A1 and CYP17A1) in endometriosis (Tsai et al., 2001; Rhee et al., 2003; Bukulmez et al., 2008a; Attar et al., 2009; Sinreih et al., 2013, 2017b; Huhtinen et al., 2014), suggesting that, in contrast to eutopic endometrium, endometriosis is able to produce steroids from cholesterol. However, it has also been argued that the presence of paracrine confounders of ovarian origin in studies using endometriomas could bias the results (Noël et al., 2011).

The contribution of STS, SULT1E1 and other SULTs was investigated by numerous studies and also in this case, conclusions are unclear (recently reviewed, Rižner, 2016). A recent investigation using 78 specimens described increased STS levels in endometriosis vs. control samples and found that the overall balance between STS and SULT1E1 differed between eutopic and ectopic tissue, implying an unbalanced flux of sulpho-conjugated estrogens in this disease (Piccinato et al., 2016b). The same research group also described an aberrant regulation of the enzymes involved in the estrogen oxidative metabolism in endometriosis (Piccinato et al., 2016a).

Although the level of the single enzymes in the intracrine machinery varies with apparently no clear association with the disease condition, the intracrinological nature of endometriosis was recently proven by comparison between serum and tissue levels of steroids in 60 patients (eutopic and ectopic endometrium) and 16 controls. Although E2 changed cyclically in eutopic tissue, E2 levels remained constant in the lesions and inversely correlated with the mRNA level of $17 \beta \mathrm{HSD} 2$ and $17 \beta$ HSD6 suggesting an impairment in E2 deactivation to E1. P levels were equal in serum and control tissues, but resulted higher in patients and correlated with high $3 \beta \mathrm{HSD} 2 \mathrm{mRNA}$. T, low in the tissue of controls, was over 13-times more concentrated at ectopic locations and correlated with low expression of SRD5A3 (Huhtinen et al., 2012a, 2014).

\section{Endometrial Cancer (EC)}

$\mathrm{EC}$ is the most common gynecological malignancy in western society and $80 \%$ of all cases are estrogen-driven (Amant et al., 2005; Morice et al., 2015). Major serum steroids are increased in patients with EC, including several substrates for intracrine E2 synthesis (Lépine et al., 2010; Audet-Walsh et al., 2011). In addition, tissue-steroid levels differ between cancer, normal tissue 
and serum and correlate with the levels of specific intracrine enzymes (see below) (Tanaka et al., 2015).

A systematic review recently explored all studies published between 1990 and 2017 assessing the expression of 17ßHSD1, 2, STS, SULT1E1, and CYP19A1, with results that describe unbalanced intracrine regulation and important inter-patient variability (Cornel et al., 2018). Most studies compared cases with controls or tumor tissue with adjacent normal endometrium. Compared with normal tissue (from controls or adjacent to tumor), $17 \beta$ HSD1 was found increased in EC (Cornel et al., 2012), decreased (Smuc and Rizner, 2009; Lépine et al., 2010) and undetected (Utsunomiya et al., 2001, 2003); 17ßHSD2 was found decreased (Utsunomiya et al., 2003, 2004) or increased (Lépine et al., 2010; Cornel et al., 2012; Sinreih et al., 2013); AKR1C3/17ßHSD5 was found unchanged (Cornel et al., 2012; Sinreih et al., 2013), increased (Ito et al., 2016) and decreased (Zakharov et al., 2010); 17ßHSD7 both decreased (Smuc and Rizner, 2009) and unchanged (Lépine et al., 2010; Cornel et al., 2012) and 17ßHSD12 was unchanged (Smuc and Rizner, 2009; Cornel et al., 2012) or increased in tumors vs. controls (Lépine et al., 2010). One recent report described decreased 17ßHSD14 levels in tumor compared with adjacent tissue (Sinreih et al., 2017a). Controversial results apply to CYP19A1, described as increased (Watanabe et al., 1995; Utsunomiya et al., 2001, 2004; Smuc and Rizner, 2009) and unchanged (Jongen et al., 2005; Pathirage et al., 2006; Cornel et al., 2012). STS/SULT1E1 expression is also inconsistent in different studies (recently reviewed in Mueller et al., 2015; Rižner, 2016).

Recent studies exploring the association between enzyme levels and tumor characteristics found a correlation between STS with tumor grade and lymphovascular invasion (Sinreih et al., 2017a) and described an association between high CYP19A1 or $17 \beta$ HSD1 and poor patient prognosis (Segawa et al., 2005; Cornel et al., 2017).

Other investigations emphasized the potential antiestrogenic and protective roles of androgens and P. Formation of DHT (via conversion of A4 to T by AKR1C3/17 $\beta$ HSD5 and of T to DHT by SRD5As) has potential antiestrogenic action because it devoids tissue from $\mathrm{T}$ (substrate of CYP19A1 yielding E2) and because it has direct endometrial antiproliferative effects via AR (Ito et al., 2016). Similar to the AKR1C3/17 $\beta$ HSD5 data reported earlier, results on SDR5A expression are inconclusive as SRD5A2 was down-regulated in a study on 47 tumor specimens compared with adjacent normal tissue (Sinreih et al., 2013), but both SRD5A1 and SRD5A2 resulted unchanged in another study on 122 tumors (although only five controls were studied) (Tanaka et al., 2015). This last study found however increased androgen levels (T and DHT) in tissue vs. blood. High DHT levels were restricted to samples with high SRD5A1 immunohistochemical staining. In addition, AR and SRD5A1 positivity was associated with good patient prognosis (Tanaka et al., 2015). The prognostic value of AR is confirmed by independent investigations (Tangen et al., 2016).

$\mathrm{P}$ is well-known for its antiestrogenic action, PR positivity is a good prognostic marker (Tangen et al., 2014) and P synthesis and metabolism are disturbed in EC (Sinreih et al., 2013). Interestingly, in a study on 47 tumors and adjacent normal tissues, EC had decreased StAR and CYP11A1 mRNA levels, indicative of diminished de novo steroid synthesis (Sinreih et al., 2013, 2017b). At the same time, EC showed decreased SRD5A2 and increased $17 \beta \mathrm{HSD} 2$ indicative of a diminished rate of conversion of P to $5 \alpha \mathrm{DHP}$ and of $20 \alpha \mathrm{DHP}$ to $5 \alpha$-pregnan-20-ol3 -one, but increased conversion of $20 \alpha$ DHP to P (see Figure 2).

\section{Other Endometrial/Gynecological Disorders}

Although literature is scarce, a potential role of intracrinology is postulated for ovarian cancer (Ito et al., 2016), for adenomyosis and fibroids (Rižner, 2016), for sarcoma, where CYP19A1 expression may have prognostic significance (Kitaoka et al., 2004) and among infertile women (Brosens et al., 2004).

\section{Intracrine Drug Targets}

Endometriosis: blocking the systemic estrogen signaling via $\mathrm{P}$, or GnRH agonist is standard care (Vercellini et al., 2014). Blocking the intracrine $\mathrm{E} 2$ generation is the future approach with on-going preclinical/clinical research.

STS inhibition showed promising results. Irosustat (Table 3) inhibited up to $100 \%$ the formation of free steroids using ex-vivo material from 27 patients (Purohit et al., 2008) and STS inhibition showed good results in a mouse model of endometriosis, where decreased size and weight of the lesions was observed (Colette et al., 2011). A phase-I clinical trial on 24 volunteers proved the safety of the STS inhibitor E2MATE (PLG2001), which reduced STS activity by over $90 \%$ and induced changes in endometrial markers (both alone or co-administered with norethindrone acetate) (Pohl et al., 2014).

Inhibitors of $17 \beta \mathrm{HSD} 1$ are in preclinical phase, and promising results are described using a primate model of endometriosis, where decreased behavior/pain symptoms were reported (Arnold and Einspanier, 2013) and using ex-vivo material from endometriosis patient (over $70 \%$ of the patients showed over $80 \%$ of enzyme inhibition) (Delvoux et al., 2014).

AKR1C3/17ßHSD5 inhibition can interfere with E2, androgen synthesis, and reduce prostaglandin-associated inflammation/proliferation and an inhibitor has recently entered a phase II trial for endometriosis (Table 3). Overall, AIs have limited efficacy for endometriosis (Ferrero et al., 2011; Dunselman et al., 2014),

EC: only in case of advanced stage/metastatic disease hormonal care is given (progestogen, tamoxifen or AIs). AIs alone have limited efficacy with low response rates (Rose et al., 2000; Ma et al., 2004; Lindemann et al., 2014). Promising data were obtained using dual regimen (AI and mTOR inhibitor; Slomovitz et al., 2015) and additional trials on combinatory regimen are on-going. STS inhibitors showed promising results in a mouse subcutaneous model of EC, with decreased tumor growth by $48-67 \%$ (Foster et al., 2008b). However, a phase II trial on advanced stage EC was stopped because of the absence of added benefit compared with progestogen treatment (Purohit and Foster, 2012; Pautier et al., 2017).

Preclinical studies on $17 \beta$ HSD1 inhibitors showed promising results in a mouse model of endometrial hyperplasia (Saloniemi et al., 2010; Järvensivu et al., 2015) and in various models of EC (Konings et al., 2018). 


\section{Endometrium: Conclusions}

The ability to synthesize DHEA from cholesterol (reported by few studies) needs confirmation. However, the endometrium possesses the enzymatic machinery to metabolize sulphatedcompounds and DHEA and form androgens and estrogens, (although this contention is wrangled by other authors: Labrie and Labrie, 2013; Labrie, 2015). Further, the endometrium can metabolize androgens and progestogens via AKR1Cs and SRD5As to produce a wide range of compounds, including estrogens (Table 6 and Figure 3). The morphological changes during the menstrual cycle are accompanied by cyclic changes in intracrine steroid and enzyme levels, indicating that steroid exposure needs to be cyclically regulated to support endometrial physiology.

\section{Gastrointestinal Tract (GIT) and Digestive System (DS)}

$\mathrm{ER} \alpha$ and ER $\beta$ are expressed throughout the GIT and DS (esophagus, stomach, colon, gallbladder, pancreas) and epidemiological studies show important influence of sex hormones in DS physiology and disturbances, with a clear gender-dependency. In the duodenum, estrogens regulate bicarbonate secretion (Nayeb-Hashemi and Kaunitz, 2009; Tuo et al., 2011). This is an important defense mechanism of the mucosa against acids discharged from the stomach, and men develop duodenal ulcer two/three-times more often than premenopausal women (Wu et al., 2008). Such estrogen protective effect is recapitulated in animal studies exposed to estrogens and anti-estrogens, and is mediated by a rapid action (i.e., non genomic) of $\mathrm{ER} \alpha$ on membrane ion channels (Smith et al., 2008).

$\mathrm{ER} \alpha, \mathrm{ER} \beta$ and GPER mediate important effects on the pancreatic beta-cells during adaptation to insulin resistance periods (e.g., pregnancy, puberty, obesity; Nadal et al., 2011). In mice, $\mathrm{ER} \alpha$ signaling regulates proliferation of beta-cell during development and after injury (Yuchi et al., 2015).

Men are also more likely than women to develop cancer in the esophagus, stomach and colon. Accordingly, estrogen treatment for prostate cancer decreases the incidence of gastric cancer and menopausal status in women is associated with colorectal cancer CRC risk (Freedman et al., 2007; Kennelly et al., 2008; Hogan et al., 2009; Duell et al., 2010). ER $\beta$ results oncoprotective at several GIT sites (Kennelly et al., 2008; Barzi et al., 2013; Caiazza et al., 2015) and low expression correlate with high CRC stage in mice and with poor differentiated gallbladder cancer in humans (Hogan et al., 2009).

The association between estrogens and DS cancer risk is however controversial. The Women's Health Initiative and other large studies showed that combined estrogens plus $\mathrm{P}$ hormone replacement therapy (HRT) decreases CRC risk, but increases that of gallbladder. In addition, CRC during HRT has a higher grade (Kennelly et al., 2008; Hogan et al., 2009; Rennert et al., 2009; Foster, 2013; Mueller et al., 2015). However, a recent randomized, placebo-controlled trial enrolling over 10,000 women receiving estrogens alone vs. placebo found no difference in CRC incidence (Lavasani et al.,
2015). Such complexity is recapitulated in animal studies where estrogens and androgens can have distinct and opposite effects on colitis and CRC (Amos-Landgraf et al., 2014; Heijmans et al., 2014). Overall, the association between DS disturbances/cancers with estrogens depends on the moment in life, extent and nature (endogenous or exogenous) of exposure and is influenced by the relative balance of the receptors (Foster, 2013). Similarly, androgens influence DS pathophysiology via complex and unclear mechanisms involving classical, membrane signaling, level of free and SHBG bound T (Roshan et al., 2016).

The lack of clear conclusion and the fact that the levels of circulating endogenous estrogens in women do not influence CRC risk indicates that intracrine steroids may have a predominant role irrespective of their circulating levels (Sato et al., 2009; Falk et al., 2015).

\section{Intracrinology in Healthy GIT-Systematic Search}

In total, 29 original papers were retrieved that described the levels of the intracrine enzymes in the GIT, published from the late 80's (Table 7 and Supplemental panel: "Systematic Review").

\section{Stomach intracrinology.}

The stomach is an endocrine tissue, and in rodents it produces steroids starting at birth and throughout adulthood (Kobayashi et al., 2013). Human gastric mucosa expresses 17 $\beta$ HSD1, 2, 12 and AKR1C3/17ßHSD5 (Table 7). The mRNA for 17ßHSD2 in mucosa surface and glandular epithelium inversely correlates with age in both genders (Oduwole et al., 2003a). Luminal gastric mucosa has strong AKR1C3/17 $\beta$ HSD5 immunoreactivity that decreases toward the gastric pits (Chang et al., 2013). Weak immunoreactivity for $17 \beta$ HSD12 localizes in the fundic glands and in the squamous epithelium of the esophagus (Sakurai et al., 2006).

Sulphatases in parietal cells of the gastric glands have a protective role in detoxification. Estrogenic SULT1E1 is not expressed whereas data for SULT2A1 are inconsistent. SULT2A1 was detected in the gastric mucosa in a study on seven subjects (Tashiro et al., 2000), but it was low/absent in other studies on 39 (Teubner et al., 2007) and 23 subjects (Chen et al., 2003).

\section{Small intestine: duodenum-jejunum-ileum.}

Due to its high exposure to food components and harmful xenobiotics, the duodenum expresses several phase I/II enzymes including DHEA/estrogenic SULT1E1, 2A1, 1A1 (Table 7). Protein and enzyme activity of SULT1E1 and 2A1 are present in human jejunum and ileum but absent in duodenum (Teubner et al., 2007), mRNA and protein levels vary with no relation either with age or gender (Her et al., 1996; Nishimura and Naito, 2006). In a study on 23 subjects, SULT1E1 and 2A1 varied inter-individually and between different intestine tracts (Chen et al., 2003). The duodenal mucosa expresses 17ßHSD2, but not 17ßHSD1 (Casey et al., 1994; Oduwole et al., 2003a) and shows strong luminal AKR1C3/17ßHSD5 (Chang et al., 2013) and weak 17ßHSD12 immunoreactivity (Sakurai et al., 2006) that decreases toward the Brunner's gland (Chang et al., 2013). 


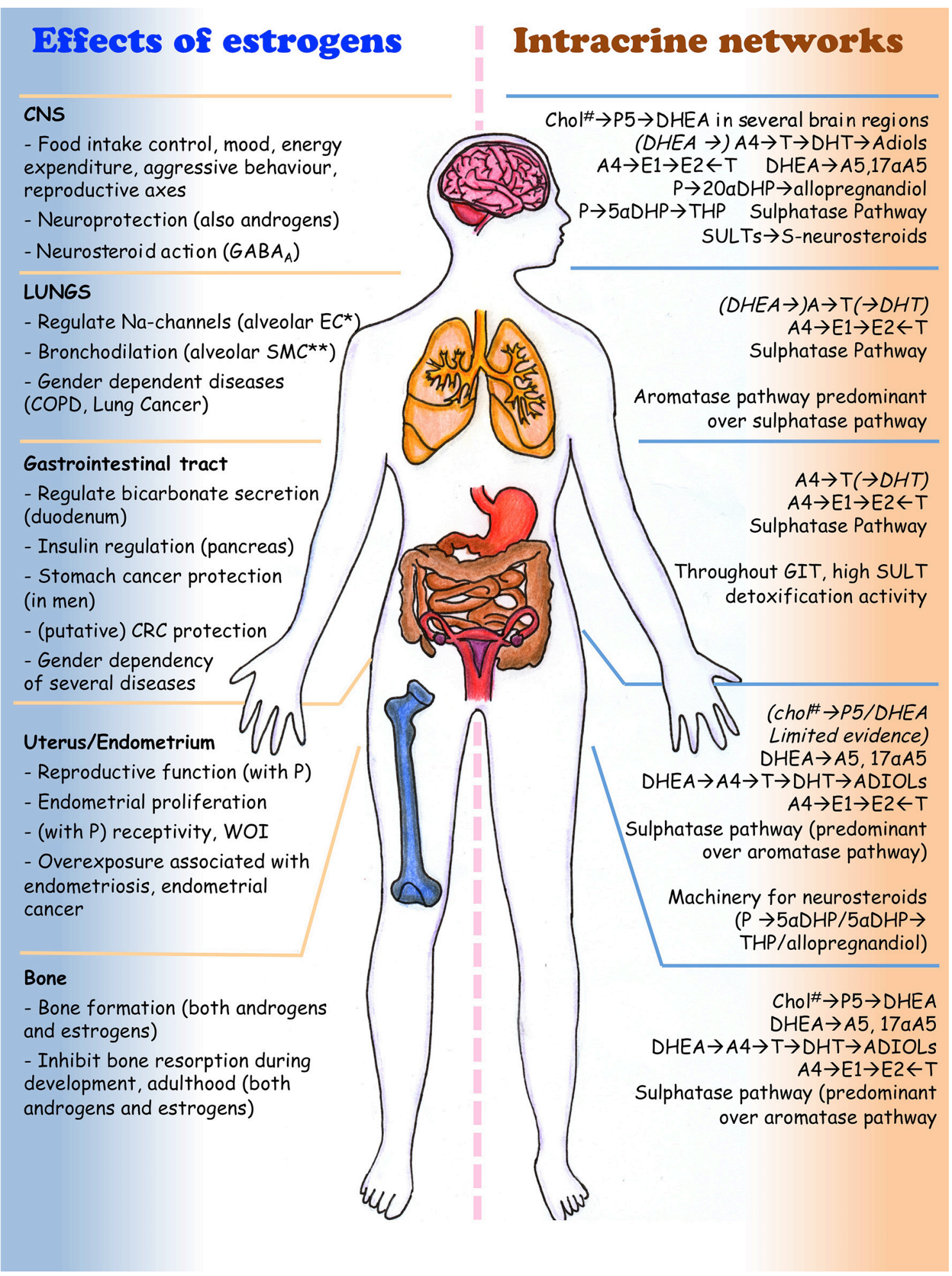

FIGURE 3 | Effect of steroids (mainly estrogens) and intracrine networks in central nervous system, lungs, digestive system, uterus and bone. Italics and by brackets are those metabolism/reactions that need conformation by independent authors (because validated at the mRNA level only or in few studies). ${ }^{*}$ EC, epithelial cells; ${ }^{*}$ SMC, smooth muscle cells; \# Chol, cholesterol. The drawing was kindly generated by Dr. Margaretha A. Skowron (Department of Urology, University Düsseldorf, Germany) for this review. 


\section{Large intestine: Colon, Cecum, Rectum}

The intracrinology of healthy colon mucosa and its relation to CRC was recently reviewed (Foster, 2013). Studies dating from 1987 demonstrated the presence of CYP19A1, 17ßHSD reductive and oxidative enzymatic activities, plus the expression of 17ßHSD1, 2, 4, CYP19A1, STS and SULT1E1 (Table 7). Most $17 \beta$ HSDs tend to have higher levels at the surface than in cryptal epithelial cells as indicated for 17ßHSD2 mRNA (Oduwole et al., 2002; Foster, 2013), and for the immunoreactivity of AKR1C3/17ßHSD5 (very strong; Chang et al., 2013) and 17ßHSD12 (weak; Sakurai et al., 2006).

\section{Pancreas}

Radiolabelled substrates demonstrated the presence of CYP19A1 and SRD5A activities in human pancreatic tissue (Iqbal et al., 1983), which expresses 17BHSD2, 12, STS, SULT1E1 (Casey et al., 1994; Miki et al., 2002; Sakurai et al., 2006; Dalla Valle et al., 2007). High levels of AKR1C3/17ßHSD5 localized in pancreatic ductules (acini and islets of Langerhans resulted negative; Chang et al., 2013).

\section{Association With Diseases}

SNPs in genes controlling estrogen synthesis, response and deactivation are associated with GIT cancers (Freedman et al., 2009; Cho et al., 2012; Zeng et al., 2016) and AKR1C4 is a candidate gene in hereditary CRC (Gylfe et al., 2013; Table 5). Also variations in the expression of these genes associate with GIT disturbances. Low 17ßHSD10 levels are associated with aberrant butyrate $\beta$-oxidation and ulcerative colitis (De Preter et al., 2012). The epithelial 17ßHSD2 level is low in case of stomach, duodenal cancer and chronic gastritis, though it is high in regenerating epithelium close to active gastritis and ulcers (Oduwole et al., 2003a). In a study on 34 gastric tumors and adjacent healthy tissue, the mRNA and protein levels of 17ßHSD2 and AKR1C3/17ßHSD5 were down-regulated in cancer (Frycz et al., 2015, 2016). Some studies showed lower oxidative 17ßHSD activity and mRNA level of 17ßHSD2 (and 4) in CRC vs. adjacent normal tissue, suggesting a protective role of estrogen deactivation. However, another study on 35 women and 39 men found that high $17 \beta \mathrm{HSD} 2$ levels were associated with poor prognosis in female patients with distal CRC (reviewed in Foster, 2013). Also 17ßHSD1 level measured by RT-qPCR and western blotting in specimens from 52 patients was lower in CRC than adjacent normal mucosa (Rawłuszko et al., 2011). CRC show also higher CYP19A1 mRNA compared with adjacent normal mucosa $(n=31)$ (Sato et al., 2012).

Although no clear target for drugs has been identified in the intracrine network, intracrine enzymes showed some values as biomarkers. In CRC, high STS/SULT1E1 ratio correlates with poor prognosis (Foster, 2013) and AKR1C3/17ßHSD5 expression with lymph-node metastasis (Nakarai et al., 2015). In addition, AKR1C1 and AKR1C3/17ßHSD5 associate with cisplatin resistance in CRC, hence inhibitors of these AKR1Cs may be used to re-sensitize patients to chemotherapy (Matsunaga et al., 2013). In a study were the levels of E1, E2 and DHEA-S were measured in CRC specimens and adjacent normal mucosa of men and women by LC-MS, intra-tumor estrogens were elevated and (in particular E1) correlated with poor prognosis. In line with an unfavorable role of intra-tissue estrogens, absence on STS was associated with long survival (Sato et al., 2009).

\section{GIT: Conclusions}

Human GIT/DS is unable to metabolize cholesterol and there is no clear evidence that it expresses $3 \beta$ HSDs, hence DHEA cannot be used to generate androgens and estrogens (Table 7 and Figure 3). Several SULTs are expressed throughout GIT and involved in detoxification and STS is regulated by estrogen in vitro via non-classical GPER signaling (Gilligan et al., 2017).

The role of steroids in pathology is complex, with divergent effects that depend on time, length and extent of exposure. In line with this, intracrine networks have unclear roles in pathogenesis. In the GIT these networks are strongly involved in the metabolisms of fatty acids and bile acids (outside the scope of this review).

\section{Bone Tissue and Skeletal System}

Bones consist of mineralized connective tissue with structural and supportive functions. The hard exterior part (cortical bone) and the trabecular and spongy cancellous tissue filling the bone interior are identical but differ in the level of mineralization. Osteoblasts, derived from multipotent mesenchymal stem cells, build the bone tissue through deposition of Type-I collagen and through the release of ions that combine chemically forming the bone mineral. Osteoclasts differentiate from hematopoietic stem cells and cause resorption of the mineralized bone mass. The balance between osteoblasts and osteoclasts regulates mineral deposition and resorption. Sex steroid hormones contribute to control bone development during puberty, contribute to bone physiology, bone mass maintenance and regulate the rate of mineral bone deposition and resorption (Svoboda et al., 2010).

The presence of the ERs as well as other hormone-receptors in normal osteoblastic cells, osteoclasts and osteoblasts is documented (Gruber et al., 2002) and estrogens and androgens stimulate bone formation and inhibit bone resorption in both males and females. During human puberty and throughout adulthood, E2 and $\mathrm{T}$ induce osteoblast proliferation (Kassem et al., 1998), which is mediated by IGF and GH (Riggs et al., 2002; Svoboda et al., 2010). Such human effects are well recapitulated in animal models. ER $\alpha-\mathrm{KO}$ (Vidal et al., 2000) and CYP19A1$\mathrm{KO}$ mice (Oz et al., 2000) exhibit low BMD in both genders and E2 treatment rescues the CYP19A1-KO phenotype (Miyaura et al., 2001). Additionally, ovariectomy stimulates osteoclast differentiation through (indirect) increased levels of IL-1, 6 and TNF in osteoblasts and other bone-derived stromal cells (Gruber et al., 2002; Svoboda et al., 2010).

Accelerated bone loss and increased osteoporotic fractures are associated with postmenopausal estrogen deficiency and low sex steroid levels elicit similar manifestations in men (Compston, 2001; Riggs et al., 2002; Syed and Khosla, 2005). Free E2 levels are associated with low lumbar spine and femoral neck bone mineral density (BMD) in both genders (Zarrabeitia et al., 2007) and estrogen therapy reduces bone loss and the risk of fracture in women with osteoporosis (Gruber et al., 2002). 
TABLE 7 | Expression of intracrine enzymes in the gastrointestinal tract (GIT)-results of the systematic search.

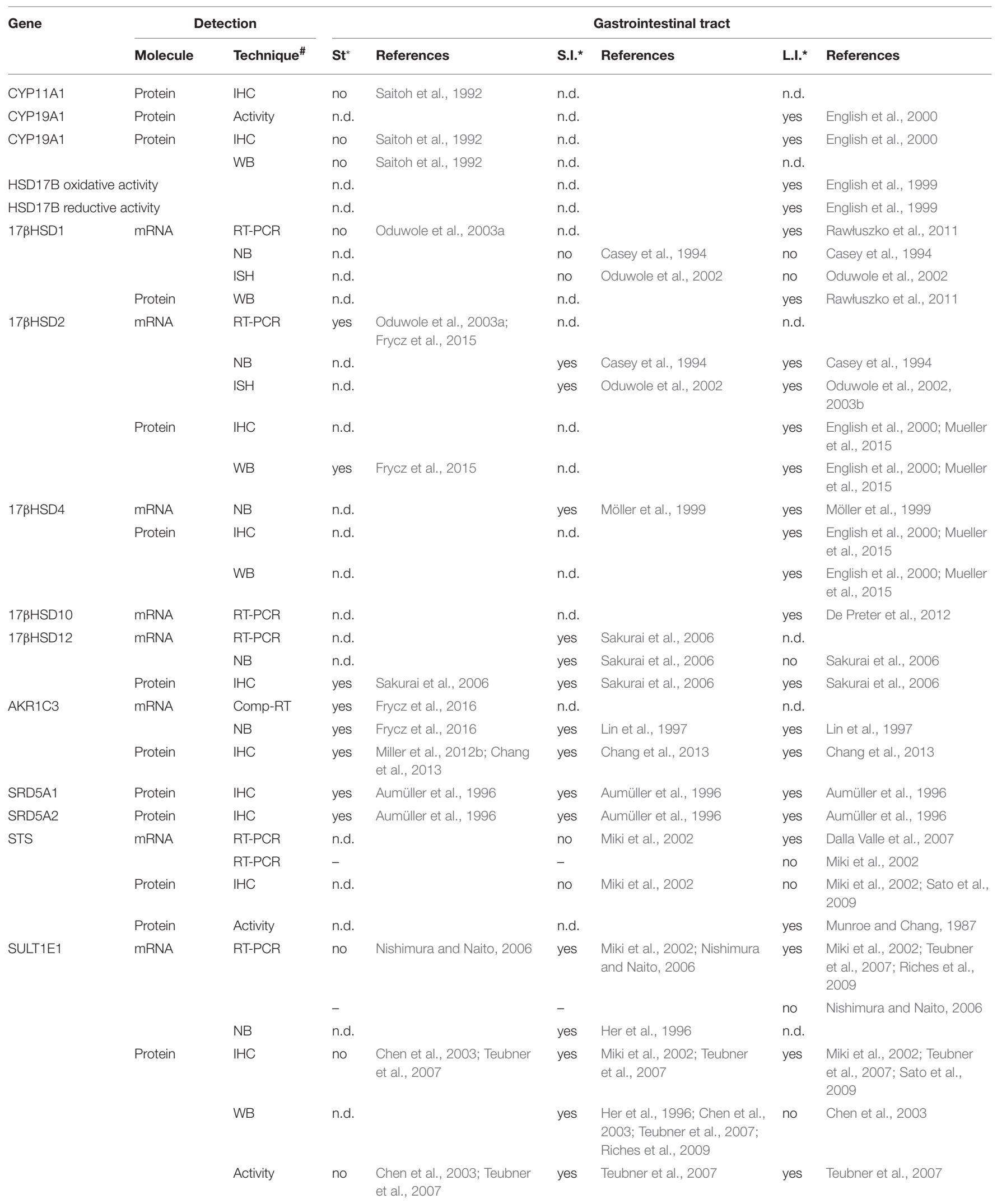


TABLE 7 | Continued

\begin{tabular}{|c|c|c|c|c|c|c|c|c|}
\hline \multirow[t]{2}{*}{ Gene } & \multicolumn{2}{|c|}{ Detection } & \multicolumn{6}{|c|}{ Gastrointestinal tract } \\
\hline & Molecule & Technique $\#$ & $\mathbf{S t}^{\star}$ & References & S.I.* & References & L.I.* & References \\
\hline \multirow[t]{8}{*}{ SULT2A1 } & mRNA & RT-PCR & no & Nishimura and Naito, 2006 & yes & Nishimura and Naito, 2006 & no & Nishimura and Naito, 2006 \\
\hline & & ISH & yes & Tashiro et al., 2000 & n.d. & & n.d. & \\
\hline & & NB & n.d. & & yes & Her et al., 1996 & n.d. & \\
\hline & Protein & $\mathrm{IHC}$ & no & Teubner et al., 2007 & yes & Teubner et al., 2007 & yes & Teubner et al., 2007 \\
\hline & & WB & yes & $\begin{array}{l}\text { Tashiro et al., 2000; Chen } \\
\text { et al., } 2003\end{array}$ & yes & $\begin{array}{l}\text { Her et al., 1996; Chen et al., } \\
\text { 2003; Teubner et al., 2007; } \\
\text { Riches et al., } 2009\end{array}$ & yes & $\begin{array}{l}\text { Chen et al., 2003; Teubner } \\
\text { et al., 2007; Riches et al., } \\
2009\end{array}$ \\
\hline & & WB & n.d. & & n.d. & & no & Chen et al., 2003 \\
\hline & & Activity & yes & Tashiro et al., 2000 & yes & $\begin{array}{l}\text { Chen et al., 2003; Teubner } \\
\text { et al., } 2007\end{array}$ & yes & $\begin{array}{l}\text { Chen et al., 2003; Teubner } \\
\text { et al., } 2007\end{array}$ \\
\hline & & Activity & no & Teubner et al., 2007 & - & & - & \\
\hline \multirow[t]{4}{*}{ SULT1A1 } & mRNA & RT-PCR & yes & Nishimura and Naito, 2006 & yes & Nishimura and Naito, 2006 & yes & Nishimura and Naito, 2006 \\
\hline & Protein & $\mathrm{IHC}$ & yes & Teubner et al., 2007 & yes & Teubner et al., 2007 & yes & Teubner et al., 2007 \\
\hline & & WB & yes & Teubner et al., 2007 & yes & $\begin{array}{l}\text { Teubner et al., 2007; Riches } \\
\text { et al., } 2009\end{array}$ & yes & $\begin{array}{l}\text { Teubner et al., 2007; Riches } \\
\text { et al., } 2009\end{array}$ \\
\hline & & Activity & yes & Teubner et al., 2007 & yes & Teubner et al., 2007 & yes & Teubner et al., 2007 \\
\hline \multirow[t]{2}{*}{ SULT2B1 } & mRNA & RT-PCR & no & Nishimura and Naito, 2006 & yes & Nishimura and Naito, 2006 & no & Nishimura and Naito, 2006 \\
\hline & & NB & n.d. & & n.d. & & no & Meloche and Falany, 2001 \\
\hline
\end{tabular}

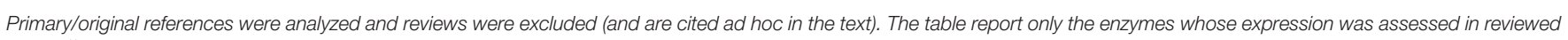
studies ${ }^{* *}$.

"St: stomach; S.I.: small intestine; L.I.: large intestine.

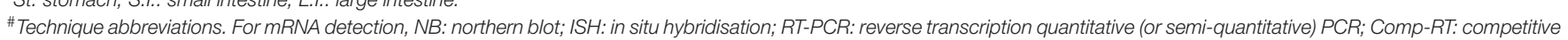
RT-PCR assay; NB: northern blotting. For protein detection: IHC: immunohistochemistry; WB; western blotting; activity: enzyme activity measurement.

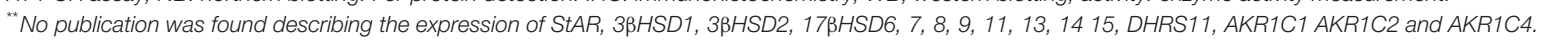
n.d.: not determined.

\section{Intracrinology in Healthy Bone-Systematic Search}

Bone expresses CYP19A1 and 17ßHSD1, and mRNA in situ hybridisation and immunohistochemistry signals were seen in lining cells, osteoblasts, chondrocytes of articular cartilage, and adipocytes adjacent to bone trabeculae in both male and female tibiae. CYP19A1 mRNA was also widely present in various bones (ribs, femurs) with inter-individual variability, but no relation with gender or age (Sasano et al., 1997). STS and $17 \beta \mathrm{HSD}$ activities were demonstrated by recovery of $[3 \mathrm{H}] \mathrm{E} 1$ and [3H]E2 after incubating femur-head fragments with [3H]E1-S (15 women and 12 men with osteoarthritis indicated for hip replacement). No gender-related differences were observed and E2 formation from androgens was lower than that from E1$\mathrm{S}$, indicating a predominant role of the sulphatase pathway in bone estrogen supply (Muir et al., 2004). Subsequent studies also demonstrated the presence of CYP11A1, CYP17A1, 17 $\beta$ HSD reductive and oxidative activity in bone tissues (Table 8). Overall, however, only six papers describing the level of intracrine enzymes in bone tissues were retrieved by the systemic search (Table 8) and most studies on bone intracrinology used in vitro cell cultures. In vitro studies were not included in our systematic review, but those on bone are briefly described in the next paragraph. These studies demonstrate the presence of a complex intracrine networks.

\section{Bone Intracrinology: In vitro and in vivo}

From early ' $90 \mathrm{~s}$, various isotopic techniques demonstrated the

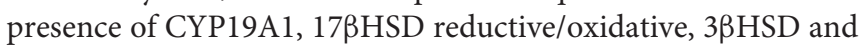

STS activities and the mRNA expression of $17 \beta$ HSD1, 2, 4, STS, SULT1E1, CYP19A1 and SDR5A in human osteoblastic (e.g., HOS, U20S, HTB-96 and MG63) and osteosarcoma cell lines like CRL-1543 (Purohit et al., 1992; Fujikawa et al., 1997; Jakob et al., 1997; Dong et al., 1998; Saito and Yanaihara, 1998; Janssen et al., 1999; Muir et al., 2004; Svoboda et al., 2007; Dias and Selcer, 2014).

In vitro evidence using osteoblastic cells show that E2 has mitogenic effects, which is blocked by the $\mathrm{ER} \alpha$ antagonist fulvestrant. Since both E1-S and DHEA-S elicit effects similar to E2, which are blocked by STS inhibition (Selcer and Difrancesca, 2012; Dias and Selcer, 2014), these studies demonstrate that conjugated steroids are activated and that DHEA is converted to E2. Studies in rat osteoblast with [14C]T demonstrated that $\mathrm{T}$ is converted by SRD5As and AKR1Cs to $3 \alpha / 3 \beta$ DIOLs, which induce proliferation via activation of ER (and not AR) (Enríquez et al., 2013).

In vitro models of osteoblast differentiation showed that various differentiation stages are accompanied by declines in STS, CYP19A1 and 17ßHSD1 (Janssen et al., 1999; Dias and Selcer, 2016).

In rats, during and after sexual maturation, in situ hybridization showed that $\operatorname{ER} \alpha$ and $\operatorname{ER} \beta$ localize in osteoblasts, osteoclasts and osteocytes covering the tibia metaphysis (responsible for elongation of long bones), and co-localize with STS. Starting at sexual maturation (e.g., 7-week-old), ERs also co-localize with CYP19A1, 17ßHSD1, 2 and SRD5A1 (van der Eerden et al., 2004). In addition, male transgenic mice 
overexpressing $17 \beta$ HSD2 show disturbed IGF-I/steroid actions in bone, with growth retardation, decreased bone formation at prepuberty and decreased serum levels of IGF-I, osteocalcin and T (Shen et al., 2008).

\section{Diseases and Treatments}

Genetic variants of estrogen and intracrine pathways are associated with bone disturbances (Table 5). Defects in the CYP19A1 and ER $\alpha$ are associated with low BMD and other skeletal disturbances (e.g., high stature, delayed bone age) and estrogen therapy ameliorates some bone abnormalities caused by CYP19A1 deficiencies in men (Smith et al., 1994; Morishima et al., 1995; Carani et al., 1997; Mullis et al., 1997; Bilezikian et al., 1998). In lumbar vertebrae, CYP19A1 levels correlate with changes in osteoporotic degree (Sasano et al., 1997).

Inhibitors of $17 \beta \mathrm{HSD} 2$ attracted attention as potential drugs to oppose the effects of low E2 on BMD, fracture and osteoporosis. Ovariectomised female macaques receiving a $17 \beta$ HSD2 inhibitor display desirable bone balance, bone strength and lower bone resorption compared with untreated controls (Bagi et al., 2008). Several compounds targeting this enzyme have been developed and their use and challenges in osteoporosis were recently reviewed (Soubhye et al., 2015).

In a study on 35 chondrosarcoma biopsies (a malignant bone cancer occurring in middle aged patients), ER $\alpha$ (mRNA and IHC) and CYP19A1 (mRNA and activity) were demonstrated in the majority of the samples, and the AI exemestane impaired the E2and androgen-induced proliferation of primary chondrosarcoma cells (Cleton-Jansen et al., 2005). Although AIs were proposed as novel drugs to treat this condition (Bovée et al., 2005), a pilot study on six patients with progressive disease showed no benefit of exemestane in progression-free survival compared with untreated patients (Meijer et al., 2011).

In a study of 28 osteosarcoma specimens (one of the most common bone cancers developing at young age) strong ER $\beta$ and PR immunoreactivity was seen in over $80 \%$ of the samples (and also correlated with $\mathrm{Ki67}$ ). $\mathrm{ER} \alpha$ and AR staining was seen in $30 \%$ of the samples, whereas CYP19A1 was undetected (Dohi et al., 2008). In another study, 20 osteosarcoma specimens, including 11 good responders to chemotherapy and nine poor responders, were subjected to cDNA microarray and 17 $\beta$ HSD10 resulted unregulated in the poor responder group. Results were further confirmed by IHC on 69 archival biopsies, hence targeting $17 \beta$ HSD10 may be a valuable approach for drug (re)sensitisation (Salas et al., 2009).

Additional intracrine imbalances are described in bone diseases, such as higher androgen reducing $17 \beta \mathrm{HSD}$ activity in benign vs. malignant tumors, declines of CYP19A1 from normal bone to osteosarcoma and expression of SULT1E1 in the majority of the skeletal benign and malignant lesions, originated in bones or from primary tumors elsewhere (Svoboda et al., 2007).

\section{Bone Tissue: Conclusions}

In vitro, animal and human studies show that intracrinology controls bone development, benign and malignant conditions, and offer novel potential drug targets (Table 8 and Figure 3). Steroids can be synthesized in situ from cholesterol (RodríguezSanz et al., 2015) and can be recruited from the serum via the sulphatase pathway. DHEA is substrate for androgen and estrogen production. The action of androgens is partly mediated by their conversion to estrogens via CYP19A1 or to estrogenic 3 $\alpha / \beta$ DIOLs (Vanderschueren et al., 2008).

\section{Lungs}

Sex steroids play an important role in lung development and homeostasis. Androgens, progestogens and estrogens are present and exert genomic and non-genomic actions via their hormonereceptors. Classical ERs (with ER $\beta$ as predominant form) and membrane GPER are expressed (Couse et al., 1997; Prossnitz and Barton, 2011; Konings et al., 2017). Sex steroids remain active in the lungs throughout lifetime and modulate lung function in both a beneficial or detrimental way, extensively reviewed (González-Arenas and Agramonte-Hevia, 2012; Townsend et al., 2012; Sathish et al., 2015).

E2 and P regulate epithelial sodium channel expression in alveolar epithelial cells (Luo et al., 2015). In alveolar smooth muscle cells, E2 induces bronchodilation via the reduction of intracellular $\mathrm{Ca}^{2+}$ (Townsend et al., 2010).

Both human and animal studies support a promoting role for estrogens and inhibitory role for androgens in lung development and maturation. During gestation and neonatal period, AR is expressed in mesenchymal and epithelial cells. Androgens inhibit the production of surfactants, which starts later in male than in female neonatal lungs (Carey et al., 2007), but also support the developing lung during branching morphogenesis (Kimura et al., 2003).

\section{Lung Intracrinology in Lungs-Systematic Search}

Adult human lungs express CYP19A1 and most 17ßHSDs (1, 2, 4, 7, 8, 11, 12, 17ßHSD5/AKR1C3; Table 8). STS, SULT and $17 \beta \mathrm{HSD} 1,12$ and $17 \beta \mathrm{HSD} 5 / \mathrm{AKR} 1 \mathrm{C} 3$ immunoreactivity localizes in the bronchial epithelium (weak for types 1 and 12, strong for type $17 \beta$ HSD5) and alveolar macrophages (Sakurai et al., 2006; Miller et al., 2012b; Chang et al., 2013; Konings et al., 2017).

Intracrinology controls lung development and maturation as shown in various animal models (Boucher et al., 2009) and intracrine enzymes are expressed already during fetal stages. Human fetal lungs possess StAR, CYP11A1, 33HSD1 mRNA (Pezzi et al., 2003), SULT1E1 activity (Jones et al., 1992) and show $17 \beta$ HSD 1 and 2 mRNAs expression at 13 and 20 weeks of gestational age (Takeyama et al., 2000). High mRNA levels of AR, $17 \beta$ HSD 2 and $17 \beta$ HSD5/AKR1C3 in mid-late gestation period and adult lungs indicate the present of androgen metabolism (Simard et al., 2010). Immunoreactivity for 17ßHSD11 is detected in bronchioles of 14 and 31 weeks old fetuses, whereas other structures are negative (e.g., alveoli, ciliated epithelium, acini of the trachea). The expression of $17 \beta$ HSD11 increases during the second half of pregnancy and maintains similar patterns in neonatal (14 days) and adult lugs (Brereton et al., 2001).

\section{Intracrinology and Lung Diseases}

Altered intracrinology is involved in lung disorders already from neonatal stages toward adulthood, and SNPs in intracrine genes are associated with the onset of diseases (Zhang et al., 
2013). Higher concentration of estrogens were measured by LCMS in women with multiple-synchronous-lung adenocarcinoma compared with single adenocarcinoma (Ikeda et al., 2016) and in neoplastic tissue compared with adjacent normal lungs (Niikawa et al., 2008; Verma et al., 2013). Type 1 17ßHSD mRNA, protein and activity are present in various non-smallcell-lung-cancer (NSCLC) cell lines where the mitogenic effect of E1 is abrogated by $17 \beta$ HSD1 knockdown (Drzewiecka and Jagodzinski, 2012; Verma et al., 2013). In specimens from 48 NSCLC patients, $17 \beta$ HSD1 expression was associated with squamous cell carcinoma and stage $3 \mathrm{~A}$ disease (Drzewiecka et al., 2015). In another study on 103 NSCLC specimens, high $17 \beta$ HSD1 immunoreactivity was associated with low intratumoural E1 and high E2:E1 ratio, whereas higher 17 $\beta$ HSD2 immunoreactivity was associated with high intratumoural E1. Multivariate regression analysis also demonstrated that increased $17 \beta$ HSD1 immunoreactivity in tumors was an independent negative prognostic factor (Verma et al., 2013).

CYP19A1 is expressed in lung cancer and has potential therapeutic value (Niikawa et al., 2008; Verma et al., 2011; Siegfried and Stabile, 2014). A recent IHC study on 335 NSCLC specimens found an inverse association between CYP19A1 expression with disease specific survival (Skjefstad et al., 2016). Similar data, although restricted to women only, were confirmed in an independent study on 150 primary lung adenocarcinoma specimens, where CYP19A1 was found as the main driver of local estrogen supply (Tanaka et al., 2016). Another study on 110 lung adenocarcinoma specimens found an association between CYP19A1 mRNA (RT-qPCR) and poor prognosis in females, never-smokers and harboring EGFR mutations (Kohno et al., 2014). However, a recent mRNA study on 96 NSCLC patients showed that CYP19A1 in combination with ER is a good prognostic marker (Aresti et al., 2014).

STS and SULT1E1 immunoreactivity is detected in the majority of NSCLC cases, and STS is a good prognostic marker (Iida et al., 2013).

Lymphangioleiomyomatosis (LAM) is a rare, potentially fatal disease affecting predominantly young women. It is strongly hormone sensitive and it is hypothesized to originate from the uterus as lung metastasis (Prizant and Hammes, 2016). The levels of ERs, PR, AR, CYP19A1, STS, 17ßHSD1 and SRD5A2 were recently assessed among 30 LAM biopsies. CYP19A1 expression resulted a useful classification marker with implication for potential therapy (Adachi et al., 2015). A recent study on specimens from 73 patients with chronic obstructive pulmonary disease (COPD) and 48 controls described an association between both CYP19A1 and 17ßHSD1 with COPD (Konings et al., 2017). CYP19A1 is also implicated in interstitial pneumonia interstitial pneumonia, where local E2 concentration and CYP19A1 activity and immunoreactivity were elevated in diseased compared with normal tissue (Taniuchi et al., 2014).

\section{Potential Novel Treatments}

Blocking the estrogen signaling showed promising preclinical results in animal models of lung cancer (Verma et al., 2011). In humans, antiestrogen treatments (ER antagonists, GnRH, oophorectomy, P) have been used in LAM (Taveira-DaSilva and Moss, 2014) and lung cancer patients (Verma et al., 2011; Kohno et al., 2014). A phase II study on advanced NSCLC patients non-responsive to platinum-based drugs tested the dual-regimen mTOR/CYP19A1 inhibitors. Unfortunately, this study was prematurely terminated due to high toxicity (Singhal et al., 2015) and one additional trial using ER antagonist plus AI (fulvestrant and anastrozole) as consolidation therapy in postmenopausal women with advanced NSCLC (NCT00932152) was terminated due to poor recruitment.

Better results were obtained using the AI letrozole as single agent or in combination with rapamycin in a phase II trial on 17 postmenopausal women with LAM (NCT01353209). AI treatment was safe and well tolerated also in the dual drug regimen (Lu et al., 2017).

\section{Lungs: Conclusions}

Steroids are involved in lung maturation, development and in susceptibility to diseases. Most $17 \beta$ HSDs, STS/SULT1E1, CYP19A1 are expressed indicating the lung's ability to metabolize androgens, estrogens and progestogens. Evidence of $3 \beta \mathrm{HSD}$ is limited to fetal tissues (Table 8 and Figure 3). Approaches aimed at decreasing local estrogens may offer future novel treatments for various lung diseases.

\section{Brain and Central Nervous System (CNS)}

One of the first CNS actions of sex steroids to be described is the hypothalamus-pituitary-gonadal axes control (Andersen and Ezcurra, 2014). The identification of steroid-receptors outside the hypothalamus, like hippocampus (controlling memory), prefrontal cortex, cerebellum and dopaminergic system regulation indicated that sex steroids have complex and widespread effects in the CNS. They control aggressive behavior, cognitive functions, mood, food intake, appetite, addiction, blood pressure, fine motor skills, motor coordination, pain circuit and both estrogens and androgens are neuroprotective (López and Tena-Sempere, 2015; Soma et al., 2015; McEwen and Milner, 2017). Estrogen deprivation in animals and humans is associated with development of metabolic disorders and estrogen administration has a general catabolic effect (López and TenaSempere, 2015). Animal experiments and KO models show that $\mathrm{ER} \alpha$ mediates the major actions of estrogens in the CNS, like the metabolic control functions (Musatov et al., 2007) and the negative-feedback on the hypothalamus-pituitary-gonadal axes (Couse et al., 2003). However, both nuclear and non-nuclear ERs are relevant in distinct CNS regions (Almey et al., 2015; López and Tena-Sempere, 2015; McEwen and Milner, 2017).

Local steroid synthesis in the CNS is demonstrated in animal studies. CYP19A1-KO mice have increased ischemic damages compared with ovariectomised wild-type mice, indicating a local action of CYP19A1 (McEwen and Milner, 2017). Similar conclusions were drawn for the estrogen protective effects on stroke, Alzheimer (AD), Parkinson diseases, aggressive behavior (Soma et al., 2015; McEwen and Milner, 2017) and mice with ablation in various $17 \beta$ HSDs show neuronal defects (Table 4). In rodents, CNS regions like the hippocampus and the hypothalamus express the enzymes involved in the local generation of steroids, like StAR, CYP11A1, CYP17A1, 3ßHSD1, 
TABLE 8 | Expression of intracrine enzymes in bone, lungs and central nervous system (CNS) - results of the systematic search.

\begin{tabular}{|c|c|c|c|c|c|c|c|c|}
\hline \multirow[t]{2}{*}{ Gene } & \multicolumn{2}{|c|}{ Detection } & \multirow[t]{2}{*}{ Bone } & \multirow[t]{2}{*}{ References } & \multirow[t]{2}{*}{ Lung } & \multirow[t]{2}{*}{ References } & \multirow[t]{2}{*}{ CNS } & \multirow[t]{2}{*}{ References } \\
\hline & Molecule & Technique $^{\#}$ & & & & & & \\
\hline StAR & mRNA & RT-PCR & n.d. & & yes $^{\wedge}$ & Pezzi et al., 2003 & n.d. & \\
\hline \multirow[t]{2}{*}{ CYP11A1 } & mRNA & RT-PCR & yes & Rodríguez-Sanz et al., 2015 & yes $^{\wedge}$ & Pezzi et al., 2003 & yes & Stoffel-Wagner, 2001 \\
\hline & & Comp-RT & n.d. & & n.d. & & yes & $\begin{array}{l}\text { Beyenburg et al., 1999; } \\
\text { Watzka et al., } 1999\end{array}$ \\
\hline & & RT-PCR & - & & - & & no & Steckelbroeck et al., 2004b \\
\hline & Protein & WB & yes $^{*}$ & Rodríguez-Sanz et al., 2015 & n.d. & & n.d. & \\
\hline & & $\mathrm{IHC}$ & n.d. & & n.d. & & no & Steckelbroeck et al., 2004b \\
\hline & & Activity & n.d. & & n.d. & & no & Steckelbroeck et al., 2004b \\
\hline \multirow[t]{5}{*}{ CYP19A1 } & mRNA & RT-PCR & yes & Oz et al., 2001 & yes & $\begin{array}{l}\text { Pezzi et al., 2003; Aresti } \\
\text { et al., 2014; Kohno et al., } \\
\text { 2014; Konings et al., } 2017\end{array}$ & yes & $\begin{array}{l}\text { Sasano et al., 1998; } \\
\text { Stoffel-Wagner et al., } \\
\text { 1998a; Stoffel-Wagner, } \\
\text { 2001; Yague et al., } 2006\end{array}$ \\
\hline & & $\mathrm{ISH}$ & yes & Sasano et al., 1997 & n.d. & & n.d. & \\
\hline & Protein & $\mathrm{IHC}$ & yes & $\begin{array}{l}\text { Sasano et al., 1997; Oz } \\
\text { et al., } 2001\end{array}$ & yes & $\begin{array}{l}\text { Verma et al., 2013; Siegfried } \\
\text { and Stabile, 2014; Taniuchi } \\
\text { et al., 2014; Konings et al., } \\
2017\end{array}$ & yes & $\begin{array}{l}\text { Naftolin et al., 1996; Yague } \\
\text { et al., 2006, } 2010\end{array}$ \\
\hline & & ELISA & n.d. & & yes & $\begin{array}{l}\text { Aresti et al., 2014; Skjefstad } \\
\text { et al., 2016; Tanaka et al., } \\
2016\end{array}$ & n.d. & \\
\hline & & Activity & yes & Schweikert et al., 1995 & yes & Taniuchi et al., 2014 & yes & $\begin{array}{l}\text { Naftolin and MacLusky, } \\
\text { 1982; Stoffel-Wagner, } 2001\end{array}$ \\
\hline 3ßHSD1 & mRNA & RT-PCR & n.d. & & yes $^{\wedge}$ & Pezzi et al., 2003 & no & Stoffel-Wagner, 2001 \\
\hline 3ßHSD2 & mRNA & RT-PCR & n.d. & & $\mathrm{no}^{\wedge}$ & Pezzi et al., 2003 & no & Stoffel-Wagner, 2001 \\
\hline & & Comp-RT & n.d. & & n.d. & & yes & Beyenburg et al., 2000 \\
\hline & mRNA & $\mathrm{ISH}$ & yes & Sasano et al., 1997 & n.d. & & n.d. & \\
\hline \multirow[t]{3}{*}{$17 \beta \mathrm{HSD} 1$} & Protein & Comp-RT & n.d. & & n.d. & & yes & $\begin{array}{l}\text { Stoffel-Wagner et al., } \\
\text { 1999b; Beyenburg et al., } \\
2000\end{array}$ \\
\hline & & $\mathrm{IHC}$ & yes & Sasano et al., 1997 & yes & $\begin{array}{l}\text { Verma et al., 2013; } \\
\text { Drzewiecka et al., 2015; } \\
\text { Konings et al., } 2017\end{array}$ & n.d. & \\
\hline & & WB & n.d. & & yes & Drzewiecka et al., 2015 & n.d. & \\
\hline \multirow[t]{5}{*}{$17 \beta \mathrm{HSD} 2$} & mRNA & RT-PCR & n.d. & & yes & $\begin{array}{l}\text { Takeyama et al., 2000; } \\
\text { Simard et al., 2010; Konings } \\
\text { et al., } 2017\end{array}$ & no & Stoffel-Wagner, 2001 \\
\hline & & Comp-RT & n.d. & & n.d. & & n.d. & $\begin{array}{l}\text { Stoffel-Wagner et al., } \\
\text { 1999b; Beyenburg et al., } \\
2000\end{array}$ \\
\hline & & NB & n.d. & & n.d. & & no & Casey et al., 1994 \\
\hline & Protein & $\mathrm{IHC}$ & n.d. & & yes & Verma et al., 2013 & n.d. & \\
\hline & & WB & & & n.d. & & n.d. & \\
\hline
\end{tabular}


TABLE 8 | Continued

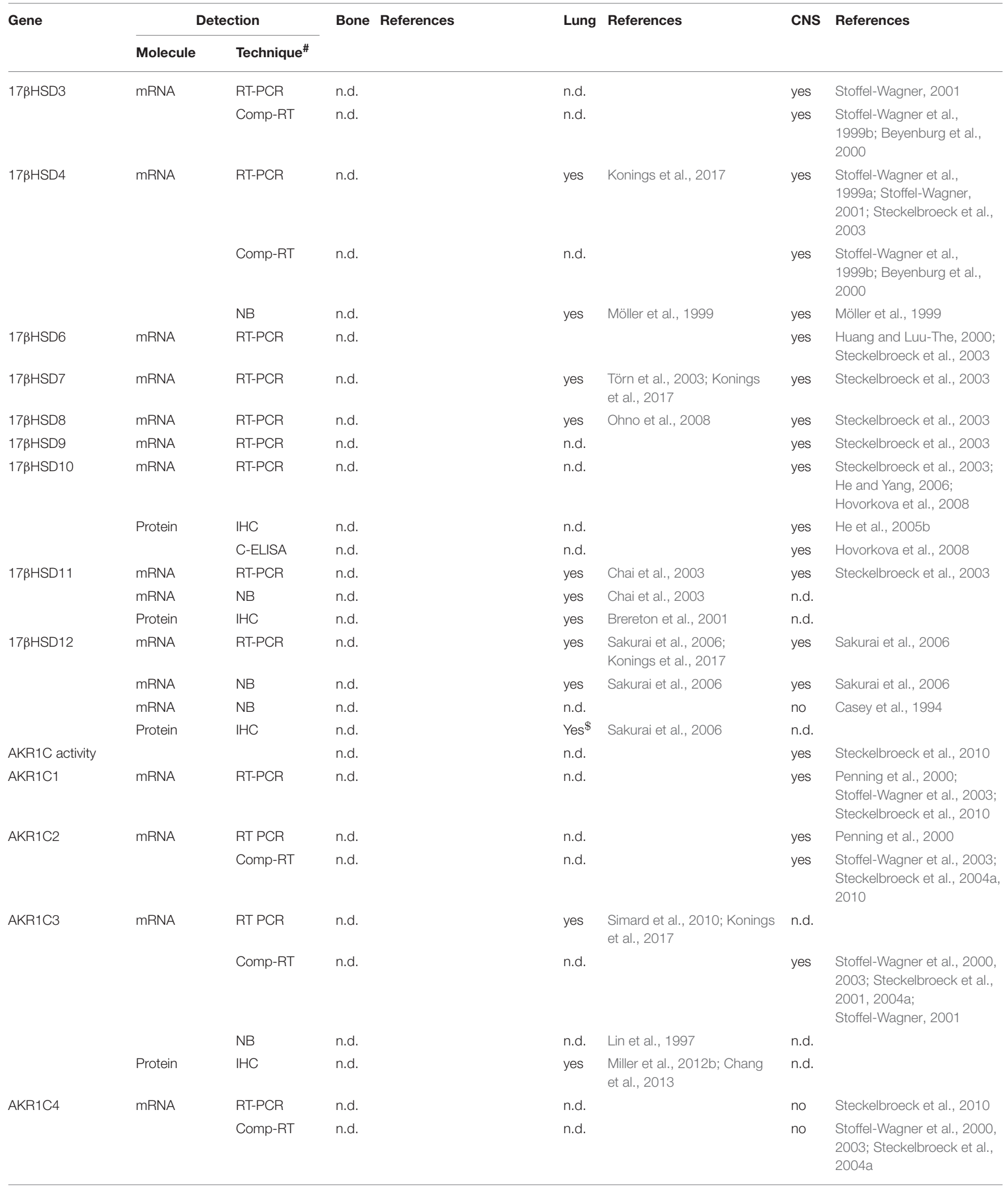


TABLE 8 | Continued

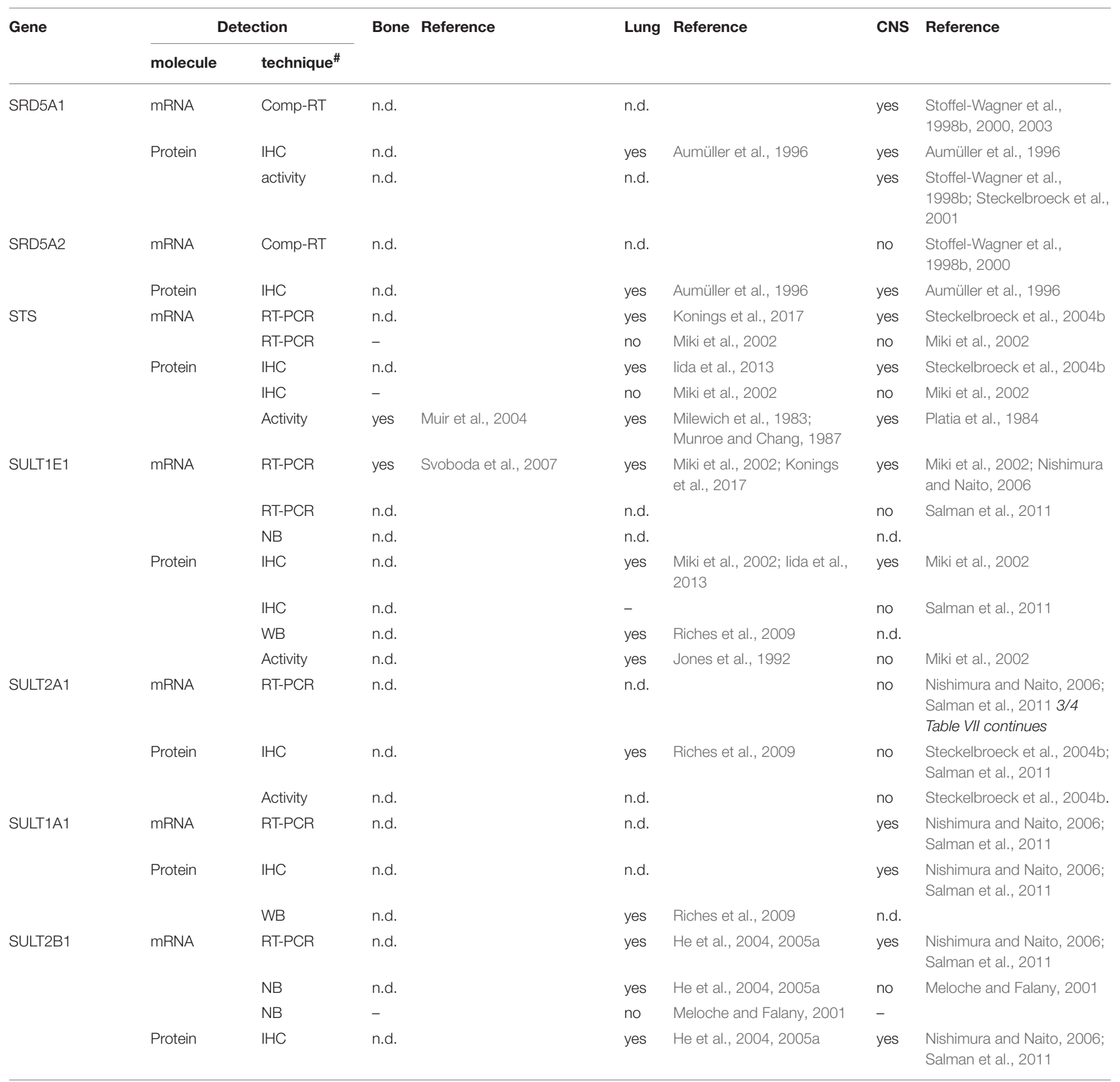

Primary/original references were analyzed and reviews were excluded (and are cited ad hoc in the text). The table report only the enzymes whose expression was assessed in reviewed studies ${ }^{* \star}$.

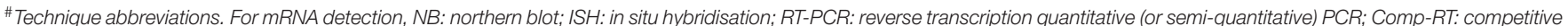
RT-PCR assay; NB: northern blotting. For protein detection: IHC: immunohistochemistry; C-ELISA: competitive ELISA assay; WB: western blotting; activity: enzyme activity measurement. "CYP11A1 and CYP17A1 activities were detected in primary cells of bone.

${ }^{* *}$ No publication was found describing the expression of 17BHSD13, 14, 15 and DHRS11.

$\wedge$ Detected in fetal lung tissue.

$\$$ IHC signal in bronchial epithelium.

CYP19A1, 17ßHSD1, SRD5A1 and 2 (mRNA and protein by immunohistochemistry or western blot), and can produce pregnenolone, DHEA, androgens and estrogens from cholesterol, as confirmed by HPLC using radiolabelled substrates and tissue cultures of brain slices (Mukai et al., 2006; Murakami et al., 2006). CYP enzymes of rat hippocampus co-localize in pyramidal neurons (CA1-CA3 regions) and granule cells (dentate gyrus) (Mukai et al., 2006; Murakami et al., 2006). 
Regulation of intracrine enzymes varies during development and sexual maturation, as indicated by mRNA expression (RT-qPCR) of 20 intracrine enzymes analyzed in rat hippocampus post-natal and throughout early (1 week) development (Kimoto et al., 2010).

Intracrinology in the CNS is particularly relevant because, beside the traditional pathway via the receptors, several steroids have neuroactivity and are allosteric modulators of $\mathrm{GABA}_{\mathrm{A}}$ receptors (Figure 2 ). Such actions are possessed also by steroids that are unable to activate the steroid hormone receptors, such as $3 \beta$ - and $3 \alpha$-hydroxyl sulpho-conjugates (P5-S and DHEAS), $5 \beta$-reduced steroids ( $5 \beta \mathrm{AN}$, etiocholanolone and $5 \beta$-THP isomers; Table 1), which are all $\mathrm{GABA}_{\mathrm{A}}$ negative modulators (in contrast to unconjugated $3 \alpha$-hydroxysteroids) (Stoffel-Wagner, 2001; Belelli and Lambert, 2005; Agís-Balboa et al., 2006; Gibbs et al., 2006; Reddy, 2010; Steckelbroeck et al., 2010).

\section{Intracrinology in CNS-Systematic Search}

Intracrine enzymes are widely expressed in human CNS (Table 8) and intratissue concentrations of steroids in distinct regions differ between regions and from the levels in the blood (Mukai et al., 2006; Murakami et al., 2006; Jäntti et al., 2010). In contrast to rodents, however, the presence of the complete steroid biosynthetic pathway is not clearly demonstrated in the human CNS and contrasting data were reported (Table 8). CYP11A1 mRNA was detected in the temporal, frontal neocortex and subcortical white matter of men, women and children (StoffelWagner, 2001). Low mRNA levels of CYP17A1 were detected in the hippocampus, amygdala, caudate nucleus, cerebellum, corpus callosum, spinal cord and thalamus (Stoffel-Wagner, 2001; Yu et al., 2002), but other authors found no expression of this enzyme (Steckelbroeck et al., 2004b, 2010; MacKenzie et al., 2008). No 3ßHSD1 or 2 was detected in temporal lobes, hippocampus, thalamus and amygdala (Stoffel-Wagner, 2001; Steckelbroeck et al., 2010), although other authors detected low levels in amygdala, caudate nucleus, cerebellum, corpus callosum, hippocampus, spinal cord and thalamus (Yu et al., 2002).

The temporal lobes (both neocortex and white matter) have $17 \beta H S D$ oxidative and reductive activities, CYP19A1 mRNA expression and activity, which is also present in hippocampus (Stoffel-Wagner et al., 1999a; Stoffel-Wagner, 2001). Temporal lobe specimens from 10 men to 12 women indicated that $17 \beta$ HSD estrogen-oxidative and DHEA-reductive metabolisms are predominant, thus producing $\mathrm{E} 1$ and $\mathrm{A} 5$, respectively (Stoffel-Wagner, 2001). Regarding the different 17ßHSDs, type 1,3 , and 4 mRNAs (but not type 2) were demonstrated by competitive reverse transcription-PCR in specimens from 34 women, 32 men and 10 children (Casey et al., 1994; Beyenburg et al., 2000). Subsequent studies confirmed the expression of types $4,7,8,10,1117 \beta$ HSD and AKR1C3/17 $\beta$ HSD5 in temporal lobes and hippocampus (Stoffel-Wagner, 2001; Steckelbroeck et al., 2003). In particular $17 \beta$ HSD10 is involved in the deactivation of THP to $5 \alpha \mathrm{DHP}$, and it is an important regulator of neurological functions (Yang et al., 2016).

Production of $5 \alpha$-androstane and pregnane neurosteroids is mediated by the action of SRD5As and AKR1Cs (Figure 2). SRD5A1 (not type 2) mRNA and enzyme activity were demonstrated in temporal neocortex and subcortical white matter, hippocampus, cerebellum, hypothalamus (Steckelbroeck et al., 2001; Stoffel-Wagner, 2001), and AKR1C4 mRNA was detected in both hippocampus and temporal lobe (StoffelWagner, 2001). AKR1C1 and AKR1C2 are widely expressed in CNS and since no specific inhibitors directed against AKR1C1 to 4 could completely inhibit AKR1C brain activity, the involvement of an unidentified enzyme is suggested (Steckelbroeck et al., $2010)$. Isomeric $5 \beta$-neurosteroids require the action of AKR1D1, and it is unknown whether AKR1D1 is expressed in CNS, or liver $5 \beta$-steroids reach peripheral regions via the circulation (Jin et al., 2011).

The sulphatase pathway in the CNS is relevant because (although recent studies are revisiting this paradigm; Qaiser et al., 2017), sulphated-steroids do not cross the blood-brain barrier. Therefore, sulphated neurosteroids like DHEA-S and P5-S need to be generated locally, and in line with this, their level in the CNS is independent from the level in the blood (Rajkowski et al., 1997) and varies throughout distinct brain regions (especially hippocampus and hypothalamus) (Jäntti et al., 2010).

STS and SULTs are widely expressed, with no gender-related differences (Table 8) (Kríz et al., 2008a,b; Mueller et al., 2015). SULT1A1 has high expression especially in specimens isolated from cerebellum, occipital and frontal lobes (Salman et al., 2009). No brain region expresses SULT2A1, whereas contrasting data exist for SULT2B1 and SULT1E1 (Table 8).

\section{Diseases and Treatments}

Steroid metabolism is deviated in schizophrenia (Bicikova et al., 2013) and aberrations and unbalances of intracrine enzymes are associated with neurological disorders (Luchetti et al., 2011 and see Table 5). In a study of 49 patients with $\mathrm{AD}$, prefrontal cortex mRNA levels of 17 $\beta$ HSD1, CYP19A1 and AKR1C2 increased at late stages (Luchetti et al., 2011). STS and SULT activities, measured by radioimmunoassay and GC-MS in 55 human brain tumor specimens, varied between tumor types (Kríz et al., 2008b). Immunoreactivity for AKR1C3/17ßHSD5 was low in medulloblastomas ( $n=10$ analyzed), high in 37 glial neoplasms and 18 meningiomas and was absent in intracranial schwannoma $(n=7)$ (Park et al., 2010). A recent screening of a chemical library of steroid inhibitors using three low grade pediatric glioma cell lines found that inhibition of $17 \beta$ HSD 3 blocked cell growth and induced apoptosis in vitro (Ajeawung et al., 2013)

Type $1017 \beta \mathrm{HSD}$ is associated with $\mathrm{AD}$ and is a potential target in diseases like $\mathrm{AD}$, Parkinson, and an X-linked mental retardation, that may arise from the impaired degradation of branched chain fatty acid, isoleucine or aberrant neurosteroid (THP) metabolism (Lim et al., 2011; Yang et al., 2016).

STS has been implicated in ADHD and a recent mouse study indicates that genetic and pharmacological manipulations of the STS axis influence the inhibitory processes and give rise to improvements in response control (Davies et al., 2014). A recent animal experiment using a model of autoimmune encephalomyelitis showed high SULT1A1 mRNA expression in laser-captured-micro-dissected white matter astrocytes, suggesting that deactivation of estrogens (and other phenolic substrates) may be responsible for the resistance to anti-neuroinflammatory treatments in these cells and could be possibly 
used as new treatments to protect CNS from inflammatory injuries (Guillot et al., 2015).

\section{CNS: Conclusions}

CNS can synthesize steroids from cholesterol, although this is restricted to few brain regions. Steroid metabolism in the CNS is particular complex due to the formation of both $5 \alpha-/ \beta$-reduced and sulpho-conjugated neurosteroids (Table 8 and Figure 3 ).

\section{Intracrinology in Other Tissues and Systems}

Steroid metabolism is also important in the immune system, skin and adipose tissue. A thorough review of these systems is outside the scope of this study, however, a brief mention is given below.

\section{Immune System and Inflammation}

Beside corticosteroids, several other steroids affect the immune system and inflammation. A5 induces white blood cells and platelets production in bone marrow (Chen et al., 2004); estrogens and androgens control B-lymphocyte development in a sex-dependent way and modulate autoimmune diseases (McCarthy, 2000; Calippe et al., 2010; Sakiani et al., 2013).

Lipopolysaccharide-mediated proinflammatory pathway in macrophages and NF- $\mathrm{B}$ activation are blocked by estrogens, which induce T-helper (Th) type 2 responses, whereas androgens stimulate type 1 responses (Iwasa et al., 2015). DHEA and DHEA-S also regulate the maturation of Th1 or Th2 cells. It was shown that plasma Th2 lymphocytes and its major secreted cytokine IL6 increase with age, and this is reversed in mice upon administration of DHEA or DHEA-S (Reed et al., 2005). Such effect was recapitulated in vitro by DHEA but not DHEA$S$ implicating the involvement of macrophage STS in lymphoid tissues where Th cell maturation occurs. In line with this, the effect of DHEA-S, but not DHEA, was impaired in vivo by an STS inhibitor (Reed et al., 2005). These data prompted to propose STS inhibition as a therapeutic approach for diseases associated with inappropriate immune responses and excess Th1 cytokines such as rheumatoid arthritis (Reed et al., 2005). Whether the action of DHEA is secondary to its conversion to androgens or estrogens is currently unclear. STS activity of peripheral blood leukocytes is higher in women during the follicular phase of the menstrual cycle than in women in the luteal phase or in men and it becomes highest during pregnancy, suggesting a role for $\mathrm{P}$ in regulating STS activity (Reed et al., 2005; Mueller et al., 2015). In vitro studies also demonstrated that STS activity is induced by cytokines such as IL6 and TNF (Mueller et al., 2015).

Opposite deregulation of the sulphatase pathway is seen in other chronic inflammatory diseases/cell types. Vascular smooth muscle cells show higher STS activity in women with mild atherosclerosis compared with women with severe disease (and male), whereas SULT1E1 activity is lower in women with severe disease (Mueller et al., 2015).

CYP19A1 is also expressed in macrophages (Konings et al., 2017) and KO mice have increased numbers of peripheral blood and bone marrow cells and inflammatory renal lesions (Shim et al., 2004). CYP19A1 inhibitors exacerbate the autoimmune lesions in a murine model of Sjögren syndrome and estrogen administration reverses such phenotype (Iwasa et al., 2015; Park et al., 2015). Opposite effects are observed in prostate, where elevated intracrine estrogens due to CYP19A1 overexpression induce inflammation and pre-malignant pathology (Ellem et al., 2009) as well as in adipose tissue (Reed et al., 2005).

\section{Skin}

The skin is the largest human organ and first barrier against pathogens where important immune functions interconnected with intracrine steroid metabolism take place (Slominski et al., 2013). Keratinocytes and sebocytes express ERs, intracrine enzymes, and the activity of sebaceous glands is influenced by steroids as indicated by the sebum production at andrenarche (Slominski et al., 2013). CYP17A1, CYP19A1, 17ßHSD1, 2, 3, 4 (and enzymes metabolizing corticosteroid - outside the scope of this review) are detected in human skin. Some genes are under the influence of vitamin D and sebocytes can synthesize T from adrenal precursors (Hughes et al., 1997; Thiboutot et al., 1998; Slominski et al., 2013). Low 17ßHSD oxidative metabolism characterizes sebaceous glands from skin areas prone to develop acne compared with other locations, suggesting a protective role of the oxidative metabolism against androgen excess (Fritsch et al., 2001). Sulphatase pathway is present in the skin (Reed et al., 2005; Simard et al., 2005), and genetic variants in STS and SULTs are associated with skin disturbances, most likely because of unbalanced steroid accumulation (Table 5).

\section{Adipose Tissue}

The adipose tissue is one of the most complex endocrine organs that besides secreting leptin and adiponectin, is a site of steroid metabolism, it establishes interaction with the CNS for glucose and lipid metabolism control, energy homeostasis and inflammation. The implication of sex steroids in adipose tissue is demonstrated by the different fat distribution that characterizes men and women (Mauvais-Jarvis et al., 2013; Varlamov et al., 2014; López and Tena-Sempere, 2015, 2016; Palmer and Clegg, 2015). ER-KO and CYP19A1-KO mice develop obesity with human-like phenotypes (López and TenaSempere, 2015). Estrogens protect against metabolic syndrome and men lacking endogenous estrogens (CYP19A1 or ER- $\alpha$ deficiency) develop hypertriglyceridemia, glucose intolerance and insulin resistance (Kim et al., 2014). In adipose tissue of men, 17ßHSD2 levels and androgen inactivation correlate with BMI (Fouad Mansour et al., 2015). A mouse study also showed that increased unsulphated-estrogen availability due to loss of SULT1E1 improved metabolic function in a model of type 2 diabetes, which leads to speculations about a potential role of SULT1E1 inhibition for this disease - at least in women (Gao et al., 2012).

Fat consists of different tissue types (white and brown) and different regional depots with distinct physiological, intracrinological characteristics and distinct relations with pathologies and metabolic disorders (Blouin et al., 2009; Mauvais-Jarvis et al., 2013). White adipose tissue is mainly subcutaneous (abdomen) or visceral (surrounding the inner organs), this last being associated with metabolic risks. A plethora of investigations demonstrated the ability of adipose tissue to 
aromatise androgens into estrogens and that the intra-tissue steroid levels are higher than the levels in blood (Bélanger et al., 2002). Androgenic and estrogenic 17 $\beta$ HSD activity and the mRNA for $17 \beta$ HSD1, 2, 3, 7, 12, AKR1C3/17 $\beta$ HSD5 were detected in both intra-abdominal and subcutaneous fat (Bélanger et al., 2002; Quinkler et al., 2004; Bellemare et al., 2009; Wang et al., 2013).

Both subcutaneous and visceral fat tissue of women expresses the androgenic $17 \beta$ HSD3 (generally considered testis specific) indicating that adipose tissue in women is substantially androgenic. Such characteristic in the visceral depot increased with increasing BMI, suggesting a link between central obesity and metabolic diseases (Corbould et al., 2002).

Additionally, several enzymes (AKR1C2, AKR1C3/17ßHSD5, CYP19A1, STS and SULT1E1) vary throughout adipocyte differentiation and maturation (Quinkler et al., 2004; Bellemare et al., 2009; Blouin et al., 2009; Mueller et al., 2015).

\section{CONCLUSIONS AND RECOMMENDATIONS}

Intracrinology consists of a complex and intricate network of alternative and redundant pathways that generate, deactivate steroids in peripheral tissues and ultimately control steroid exposure in a tissue specific manner. A number of compounds have that ability to bind and activate more than one nuclear receptors thus exerting multiple biological actions. Blood steroids represent a reservoir of substrates that support these intracrine networks. Studies retrieved by the systematic search demonstrated that most investigations rely on RT-PCR or IHC to detect enzyme and protein, and frequently without multiple-technique confirmation of the data. Since both techniques present limitations, and antibodies for IHC often perform sub-optimally (detection limit is not sufficient to detect some intracrine enzymes, crossreactivity between isoforms) these techniques are not always suitable to infer the real biological role of a reaction/enzyme.

However, the recent technological advances in steroid profiling together with an improved knowledge of intracrine enzymes and the possibility to validate data using multiple approaches (RNA, protein, activity, steroid profiling) create today unprecedented opportunities to expand our understanding of intracrinology, its relation with endocrinology and to exploit this knowledge in patient care. Improved multiplex platforms allowing to profile in peripheral tissues all steroids depicted in Figure 2 are awaited and will elucidate the relevant tissue-specific networks. It is envisaged that novel prognostic markers and drug targets will become of clinical relevance soon.

We should however be aware that the redundant actions of intracrine enzymes, their substrate promiscuity, the existence of alternative pathways and the patient-to-patient variability might result in drug insensitivity. Dual/triple inhibitors will help solving this problem. In addition, in order to optimize research on novel drugs, the classical preclinical drug discovery pipelines (safety, pharmacokinetics and dynamics), should encompass parallel research lines to learn how to pre-select potentially responsive patients.

Finally, since we know that steroidal and intracrine drugs might have profound effects on the CNS, it is desirable to have in depth research on the neurological effects of potential novel drugs during the nonclinical phase of drug development. This will facilitate to select suitable compounds to the clinical development.

\section{AUTHOR CONTRIBUTIONS}

GK drafted the study, prepared figures, tables, intermediate versions, final version and approved final version. LB drafted part of the study, contributed to intermediate versions and approved final version. KC drafted part of the study, contributed to intermediate versions and approved final version. BD contributed to intermediate versions and approved final version. TL drafted part of the study, contributed to intermediate versions and approved final version. PK contributed to intermediate versions and approved final version. $\mathrm{MB}$ contributed to intermediate versions and approved final version. RK contributed to intermediate versions and approved final version. SX drafted part of the study, contributed to intermediate versions and approved final version. AR drafted the study, prepared figures, tables, intermediate versions, final version and approved final version.

\section{FUNDING}

The present study was sponsored by the Dutch Cancer Society (KWF Kankerbestrijding: www.kwf.nl), contract number UM13-5782 granted to AR.

\section{ACKNOWLEDGMENTS}

We are grateful to Dr. Margaretha A Skowron (Department of Urology, University Düsseldorf, Germany) for graphic arts.

We also would like to thank the management and curator of the online databases we made use of, namely: the database of chemical molecules PubChem (www.ncbi.nlm.nih.gov; pubchem.ncbi.nlm.nih.gov) maintained by the National Center for Biotechnology Information (NCBI; National Library of Medicine/United States National Institutes of Health - NIH); Chemical Abstracts Service (CAS), maintained by the American Chemical Society (www.cas.org); Human Metabolome Data Base (HMDB, www.hmdb.ca), funded and maintained by Genome Canada; Chemical Book (www.chemicalbook.com), funded by industrial partners; Chemical Entities of Biological Interest (ChEBI; www.ebi.ac.uk/chebi/init.do), curated by the European Bioinformatics Institute of the European Molecular Biology Laboratory (EMBL); drug and drug target database Drugbank (www.drugbank.ca/drugs), University of Alberta and The Metabolomics Innovation Centre; GeneCards (www. genecards.org), developed and maintained by the Crown Human Genome Center at the Weizmann Institute of Science; Online Mendelian Inheritance in Man $^{\circledR}\left(\mathrm{OMIM}^{\circledR}\right.$, https://omim.org/), 
McKusick-Nathans Institute of Genetic Medicine, Johns Hopkins University (Baltimore, MD); Mouse Genome Informatics (MGI; www.informatics.jax.org), Mouse Genome Database at the Mouse Genome Informatics website, The Jackson Laboratory, Bar Harbor, Maine.

\section{SUPPLEMENTARY MATERIAL}

The Supplementary Material for this article can be found online at: https://www.frontiersin.org/articles/10.3389/fphar. 2018.00940/full\#supplementary-material

\section{REFERENCES}

Abdelsamie, A. S., van Koppen, C. J., Bey, E., Salah, M., Börger, C., Siebenbürger, L. et al. (2017). 17ß-HSD1 inhibitor with sub-nanomolar IC50 for a proof-of-principle study. Eur. J. Med. Chem. 127, 944-957. doi: 10.1016/j.ejmech.2016.11.004

Acién, P., Velasco, I., Gutiérrez, M., and Martínez-Beltrán, M. (2007). Aromatase expression in endometriotic tissues and its relationship to clinical and analytical findings. Fertil. Steril. 88, 32-38. doi: 10.1016/j.fertnstert.2006.11.188

Adachi, K., Miki, Y., Saito, R., Hata, S., Yamauchi, M., Mikami, Y., et al. (2015). Intracrine steroid production and mammalian target of rapamycin pathways in pulmonary lymphangioleiomyomatosis. Hum. Pathol. 46, 1685-1693. doi: 10.1016/j.humpath.2015.02.019

Adessi, G. L., Prost, O., Agnani, G., Petitjean, A., and Burnod, J. (1984). Estrone sulfatase activity in normal and abnormal endometrium. Arch. Gynecol. 236, 13-18. doi: 10.1007/BF02114864

Adjei, A. A., Thomae, B. A., Prondzinski, J. L., Eckloff, B. W., Wieben, E. D., and Weinshilboum, R. M. (2003). Human estrogen sulfotransferase (SULT1E1) pharmacogenomics: Gene resequencing and functional genomics. Br. J. Pharmacol. 139, 1373-1382. doi: 10.1038/sj.bjp.0705369

Aghajanova, L., Hamilton, A., Kwintkiewicz, J., Vo, K. C., and Giudice, L. C. (2009). Steroidogenic enzyme and key decidualization marker dysregulation in endometrial stromal cells from women with versus without endometriosis. Biol. Reprod. 80, 105-114. doi: 10.1095/biolreprod.108.070300

Agís-Balboa, R. C., Pinna, G., Zhubi, A., Maloku, E., Veldic, M., Costa, E., et al. (2006). Characterization of brain neurons that express enzymes mediating neurosteroid biosynthesis. Proc. Natl. Acad. Sci. U.S.A. 103, 14602-14607. doi: 10.1073/pnas.0606544103

Ajeawung, N. F., Maltais, R., Jones, C., Poirier, D., and Kamnasaran, D. (2013). Viability screen on pediatric low grade glioma cell lines unveils a novel anticancer drug of the steroid biosynthesis inhibitor family. Cancer Lett. 330, 96-105. doi: 10.1016/j.canlet.2012.11.034

Almey, A., Milner, T. A., and Brake, W. G. (2015). Estrogen receptors in the central nervous system and their implication for dopamine-dependent cognition in females. Horm. Behav. 74, 125-138. doi: 10.1016/j.yhbeh.2015.06.010

Alshogran, O. Y. (2017). Pharmacogenetics of aldo-keto reductase 1C (AKR1C) enzymes. Expert Opin. Drug Metab. Toxicol. 13, 1063-1073. doi: 10.1080/17425255.2017.1376648

Amant, F., Moerman, P., Neven, P., Timmerman, D., Van Limbergen, E., and Vergote, I. (2005). Endometrial cancer. Lancet 366, 491-505. doi: 10.1016/S0140-6736(05)67063-8

Amos-Landgraf, J. M., Heijmans, J., Wielenga, M. C., Dunkin, E., Krentz, K. J., Clipson, L., et al. (2014). Sex disparity in colonic adenomagenesis involves promotion by male hormones, not protection by female hormones. Proc. Natl. Acad. Sci. U.S.A. 111, 16514-16519. doi: 10.1073/pnas.1323064111

Andersen, C. Y., and Ezcurra, D. (2014). Human steroidogenesis: implications for controlled ovarian stimulation with exogenous gonadotropins. Reprod. Biol. Endocrinol. 12:128. doi: 10.1186/1477-7827-12-128

Andrew, A. S., Hu, T., Gu, J., Gui, J., Ye, Y., Marsit, C. J., et al. (2012). HSD3B and gene-gene interactions in a pathway-based analysis of genetic susceptibility to bladder cancer. PLoS ONE 7:e51301. doi: 10.1371/journal.pone.0051301

Aresti, U., Carrera, S., Iruarrizaga, E., Fuente, N., Marrodan, I., de Lobera, A. R., et al. (2014). Estrogen receptor 1 gene expression and its combination with estrogen receptor 2 or aromatase expression predicts survival in non-small cell lung cancer. PLoS ONE 9:e109659. doi: 10.1371/journal.pone.0109659

Arnold, C., and Einspanier, A. (2013). Medical treatment improves social behavior in a primate endometriosis model (Callithrix jacchus). J. Med. Primatol. 42, 112-119. doi: 10.1111/jmp.12042

Ashton, K. A., Proietto, A., Otton, G., Symonds, I., McEvoy, M., Attia, J., et al. (2010). Polymorphisms in genes of the steroid hormone biosynthesis and metabolism pathways and endometrial cancer risk. Cancer Epidemiol. 34, 328-337. doi: 10.1016/j.canep.2010.03.005

Attar, E., Tokunaga, H., Imir, G., Yilmaz, M. B., Redwine, D., Putman, M., et al. (2009). Prostaglandin E2 via steroidogenic factor-1 coordinately regulates transcription of steroidogenic genes necessary for estrogen synthesis in endometriosis. J. Clin. Endocrinol. Metab. 94, 623-631. doi: $10.1210 /$ jc. $2008-1180$

Audet-Walsh, É., Bellemare, J., Lacombe, L., Fradet, Y., Fradet, V., Douville, P., et al. (2012). The impact of germline genetic variations in hydroxysteroid (17beta) dehydrogenases on prostate cancer outcomes after prostatectomy. Eur. Urol. 62, 88-96. doi: 10.1016/j.eururo.2011.12.021

Audet-Walsh, E., Lepine, J., Gregoire, J., Plante, M., Caron, P., Tetu, B., et al. (2011). Profiling of endogenous estrogens, their precursors, and metabolites in endometrial cancer patients: association with risk and relationship to clinical characteristics. J. Clin. Endocrinol. Metab. 96, E330E339. doi: 10.1210/jc.2010-2050

Aumüller, G., Eicheler, W., Renneberg, H., Adermann, K., Vilja, P., and Forssmann, W. G. (1996). Immunocytochemical evidence for differential subcellular localization of 5 alpha-reductase isoenzymes in human tissues. Acta Anat. (Basel). 156, 241-252. doi: 10.1159/000147852

Bacallao, K., Leon, L., Gabler, F., Soto, E., Romero, C., Valladares, L., et al. (2008). In situ estrogen metabolism in proliferative endometria from untreated women with polycystic ovarian syndrome with and without endometrial hyperplasia. $J$. Steroid Biochem. Mol. Biol. 110, 163-169. doi: 10.1016/j.jsbmb.2008.03.031

Baes, M., Huyghe, S., Carmeliet, P., Declercq, P. E., Collen, D., Mannaerts, G. P., et al. (2000). Inactivation of the peroxisomal multifunctional protein-2 in mice impedes the degradation of not only 2-methyl-branched fatty acids and bile acid intermediates but also of very long chain fatty acids. J. Biol. Chem. 275, 16329-16336. doi: 10.1074/jbc.M001994200

Bagi, C. M., Wood, J., Wilkie, D., and Dixon, B. (2008). Effect of $17 \beta$ hydroxysteroid dehydrogenase type 2 inhibitor on bone strength in ovariectomized cynomolgus monkeys. J. Musculoskelet. Neuronal Interact. 8, 267-280.

Bair, S. R., and Mellon, S. H. (2004). Deletion of the mouse P450c17 gene causes early embryonic lethality. Mol. Cell. Biol. 24, 5383-5390. doi: 10.1128/MCB.24.12.5383-5390.2004

Balk, S. P., and Knudsen, K. E. (2008). AR, the cell cycle, and prostate cancer. Nucl. Recept. Signal. 6:e001.

Barzi, A., Lenz, A. M., Labonte, M. J., and Lenz, H. J. (2013). Molecular pathways: estrogen pathway in colorectal cancer. Clin. Cancer Res. 19, 5842-5848. doi: 10.1158/1078-0432.CCR-13-0325

Bélanger, C., Luu-The, V., Dupont, P., and Tchernof, A. (2002). Adipose tissue intracrinology: potential importance of local androgen/estrogen metabolism in the regulation of adiposity. Horm. Metab. Res. 34, 737-745. doi: $10.1055 / \mathrm{s}-2002-38265$

Belelli, D., and Lambert, J. J. (2005). Neurosteroids: endogenous regulators of the GABA(A) receptor. Nat. Rev. Neurosci. 6, 565-575. doi: 10.1038/nrn1703

Bellemare, V., Faucher, F., Breton, R., and Luu-The, V. (2005). Characterization of $17 \alpha$-hydroxysteroid dehydrogenase activity (17 $\alpha$-HSD) and its involvement in the biosynthesis of epitestosterone. BMC Biochem. 6:12. doi: 10.1186/1471-2091-6-12

Bellemare, V., Laberge, P., Noel, S., Tchernof, A., and Luu-The, V. (2009). Differential estrogenic $17 \beta$-hydroxysteroid dehydrogenase activity and type 12, 17 $\beta$-hydroxysteroid dehydrogenase expression levels in preadipocytes and differentiated adipocytes. J. Steroid Biochem. Mol. Biol. 114, 129-134. doi: 10.1016/j.jsbmb.2009.01.002 
Berstein, L., Zimarina, T., Imyanitov, E., Kovalevskij, A., Maximov, S., Pujol, P., et al. (2006). Hormonal imbalance in two types of endometrial cancer and genetic polymorphism of steroidogenic enzymes. Maturitas 54, 352-355. doi: 10.1016/j.maturitas.2006.04.017

Beyenburg, S., Stoffel-Wagner, B., Watzka, M., Blumcke, I., Bauer, J., Schramm, J., et al. (1999). Expression of cytochrome P450scc mRNA in the hippocampus of patients with temporal lobe epilepsy. Neuroreport 10, 3067-3070. doi: 10.1097/00001756-199909290-00035

Beyenburg, S., Watzka, M., Blumcke, I., Schramm, J., Bidlingmaier, F., Elger, C. E., et al. (2000). Expression of mRNAs encoding for $17 \beta$-hydroxisteroid dehydrogenase isozymes 1, 2, 3 and 4 in epileptic human hippocampus. Epilepsy Res. 41, 83-91. doi: 10.1016/S0920-1211(00)00130-3

Bicikova, M., Hill, M., Ripova, D., Mohr, P., and Hampl, R. (2013). Determination of steroid metabolome as a possible tool for laboratory diagnosis of schizophrenia. J. Steroid Biochem. Mol. Biol. 133, 77-83. doi: 10.1016/j.jsbmb.2012.08.009

Bilezikian, J. P., Morishima, A., Bell, J., and Grumbach, M. M. (1998). Increased bone mass as a result of estrogen therapy in a man with aromatase deficiency. N. Engl. J. Med. 339, 599-603. doi: 10.1056/NEJM199808273390905

Blake, J. A., Eppig, J. T., Kadin, J. A., Richardson, J. E., Smith, C. L., Bult, C. J., et al. (2017). Mouse Genome Database (MGD)-2017: community knowledge resource for the laboratory mouse. Nucleic Acids Res. 45, D723D729. doi: 10.1093/nar/gkw1040

Blouin, K., Nadeau, M., Mailloux, J., Daris, M., Lebel, S., Luu-The, V., et al. (2009). Pathways of adipose tissue androgen metabolism in women: depot differences and modulation by adipogenesis. Am. J. Physiol. Endocrinol. Metab. 296, E244-E255. doi: 10.1152/ajpendo.00039.2008

Bothe, U., Busemann, M., Steinmeyer, A., Droescher, P., Fischer, O. M., Peters, M., et al. (2017). "First time disclosure of BAY 1128688: a novel AKR1C3 inhibitor for the treatment of endometriosis," in 254th ACS National Meeting \& Exposition (Washington, DC).

Boucher, E., Provost, P. R., Plante, J., and Tremblay, Y. (2009). Androgen receptor and $17 \beta-H S D$ type 2 regulation in neonatal mouse lung development. Mol. Cell. Endocrinol. 311, 109-119. doi: 10.1016/j.mce.2009.06.012

Bovée, J. V., Cleton-Jansen, A. M., Taminiau, A. H., and Hogendoorn, P. C. (2005). Emerging pathways in the development of chondrosarcoma of bone and implications for targeted treatment. Lancet Oncol. 6, 599-607. doi: 10.1016/S1470-2045(05)70282-5

Brereton, P., Suzuki, T., Sasano, H., Li, K., Duarte, C., Obeyesekere, V., et al. (2001). Pan1b (17ßHSD11)-enzymatic activity and distribution in the lung. Mol. Cell. Endocrinol. 171, 111-117. doi: 10.1016/S0303-7207(00)00417-2

Brosens, J., Verhoeven, H., Campo, R., Gianaroli, L., Gordts, S., Hazekamp, J., et al. (2004). High endometrial aromatase P450 mRNA expression is associated with poor IVF outcome. Hum. Reprod. 19, 352-356. doi: 10.1093/humrep/deh075

BroŽic, P., Turk, S., Rizner, T. L., and Gobec, S. (2011). Inhibitors of aldo-keto reductases AKR1C1-AKR1C4. Curr. Med. Chem. 18, 2554-2565. doi: 10.2174/092986711795933713

Bukulmez, O., Hardy, D. B., Carr, B. R., Auchus, R. J., Toloubeydokhti, T., Word, R. A., et al. (2008a). Androstenedione up-regulation of endometrial aromatase expression via local conversion to estrogen: potential relevance to the pathogenesis of endometriosis. J. Clin. Endocrinol. Metab. 93, 3471-3477. doi: $10.1210 /$ jc. $2008-0248$

Bukulmez, O., Hardy, D. B., Carr, B. R., Word, R. A., and Mendelson, C. R. (2008b). Inflammatory status influences aromatase and steroid receptor expression in endometriosis. Endocrinology 149, 1190-1204. doi: 10.1210/en.2007-0665

Bulun, S. E., Economos, K., Miller, D., and Simpson, E. R. (1994). CYP19 (aromatase cytochrome P450) gene expression in human malignant endometrial tumors. J. Clin. Endocrinol. Metab. 79, 1831-1834.

Bulun, S. E., Mahendroo, M. S., and Simpson, E. R. (1993). Polymerase chain reaction amplification fails to detect aromatase cytochrome $\mathrm{P} 450$ transcripts in normal human endometrium or decidua. J. Clin. Endocrinol. Metab. 76, 1458-1463.

Caiazza, F., Ryan, E. J., Doherty, G., Winter, D. C., and Sheahan, K. (2015). Estrogen receptors and their implications in colorectal carcinogenesis. Front. Oncol. 5:19. doi: 10.3389/fonc.2015.00019

Calippe, B., Douin-Echinard, V., Delpy, L., Laffargue, M., Lelu, K., Krust, A., et al. (2010). 17 $\beta$-estradiol promotes TLR4-triggered proinflammatory mediator production through direct estrogen receptor alpha signaling in macrophages in vivo. J. Immunol. 185, 1169-1176. doi: 10.4049/jimmunol.0 902383

Carani, C., Qin, K., Simoni, M., Faustini-Fustini, M., Serpente, S., Boyd, J., et al. (1997). Effect of testosterone and estradiol in a man with aromatase deficiency. N. Engl. J. Med. 337, 91-95. doi: 10.1056/NEJM199707103370204

Carey, M. A., Card, J. W., Voltz, J. W., Arbes, S. J. Jr., Germolec, D. R., Korach, K. S., et al. (2007). It's all about sex: gender, lung development and lung disease. Trends Endocrinol. Metab. 18, 308-313. doi: 10.1016/j.tem.2007.08.003

Carneiro, M. M., Morsch, D. M., Camargos, A. F., Reis, F. M., and Spritzer, P. M. (2008). Androgen receptor and $5 \alpha$-reductase are expressed in pelvic endometriosis. BJOG 115, 113-117. doi: 10.1111/j.1471-0528.2007.01521.x

Carneiro, M. M., Morsch, D. M., Camargos, A. F., Spritzer, P. M., and Reis, F. M. (2007). Expression of 17 $\beta$-hydroxysteroid dehydrogenase type 2 in pelvic endometriosis. Gynecol. Endocrinol. 23, 188-192. doi: 10.1080/09513590701200850

Caron, K. M., Soo, S. C., Wetsel, W. C., Stocco, D. M., Clark, B. J., and Parker, K. L. (1997). Targeted disruption of the mouse gene encoding steroidogenic acute regulatory protein provides insights into congenital lipoid adrenal hyperplasia. Proc. Natl. Acad. Sci. U.S.A. 94, 11540-11545. doi: 10.1073/pnas.94.21.11540

Casey, M. L., MacDonald, P. C., and Andersson, S. (1994). 17 beta-Hydroxysteroid dehydrogenase type 2: chromosomal assignment and progestin regulation of gene expression in human endometrium. J. Clin. Invest. 94, 2135-2141. doi: 10.1172/JCI117569

Chai, Z., Brereton, P., Suzuki, T., Sasano, H., Obeyesekere, V., Escher, G., et al. (2003). 17 $\beta$-hydroxysteroid dehydrogenase type XI localizes to human steroidogenic cells. Endocrinology 144, 2084-2091. doi: 10.1210/en.2002-221030

Chang, T. S., Lin, H. K., Rogers, K. A., Brame, L. S., Yeh, M. M., Yang, Q., et al. (2013). Expression of aldo-keto reductase family 1 member C3 (AKR1C3) in neuroendocrine tumors \& adenocarcinomas of pancreas, gastrointestinal tract, and lung. Int. J. Clin. Exp. Pathol. 6, 2419-2429.

Chen, F., Knecht, K., Leu, C., Rutledge, S. J., Scafonas, A., Gambone, C., et al. (2004). Partial agonist/antagonist properties of androstenedione

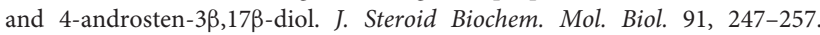
doi: 10.1016/j.jsbmb.2004.04.009

Chen, G., Zhang, D., Jing, N., Yin, S., Falany, C. N., and RadominskaPandya, A. (2003). Human gastrointestinal sulfotransferases: identification and distribution. Toxicol. Appl. Pharmacol. 187, 186-197. doi: 10.1016/S0041-008X(02)00073-X

Cho, L. Y., Yang, J. J., Ko, K. P., Ma, S. H., Shin, A., Choi, B. Y., et al. (2012). Genetic susceptibility factors on genes involved in the steroid hormone biosynthesis pathway and progesterone receptor for gastric cancer risk. PLOS ONE 7:e47603. doi: 10.1371/journal.pone.0047603

Ciuffi, M., Savino, L., Santini, D., Buzzoni, P., Scarselli, G., and Mazzei, T. (1982). Estradiol and progestin receptors, 17-beta-hydroxysteroid-dehydrogenase and histopathologic grade in endometrial carcinoma. Tumori 68, 217-221. doi: $10.1177 / 030089168206800305$

Cleton-Jansen, A. M., van Beerendonk, H. M., Baelde, H. J., Bovee, J. V., Karperien, M., and Hogendoorn, P. C. (2005). Estrogen signaling is active in cartilaginous tumors: implications for antiestrogen therapy as treatment option of metastasized or irresectable chondrosarcoma. Clin. Cancer Res. 11, 8028-8035. doi: 10.1158/1078-0432.CCR-05-1253

Colette, S., Defrere, S., Lousse, J. C., Van Langendonckt, A., Gotteland, J. P., Loumaye, E., et al. (2011). Inhibition of steroid sulfatase decreases endometriosis in an in vivo murine model. Hum. Reprod. 26, 1362-1370. doi: 10.1093/humrep/der079

Colette, S., Defrere, S., Van Kerk, O., Van Langendonckt, A., Dolmans, M. M., and Donnez, J. (2013). Differential expression of steroidogenic enzymes according to endometriosis type. Fertil. Steril. 100, 1642-1649. doi: 10.1016/j.fertnstert.2013.08.003

Colette, S., Lousse, J. C., Defrere, S., Curaba, M., Heilier, J. F., Van Langendonckt, A., et al. (2009). Absence of aromatase protein and mRNA expression in endometriosis. Hum. Reprod. 24, 2133-2141. doi: 10.1093/humrep/dep199

Compston, J. E. (2001). Sex steroids and bone. Physiol. Rev. 81, 419-447. doi: 10.1152/physrev.2001.81.1.419

Corbould, A. M., Bawden, M. J., Lavranos, T. C., Rodgers, R. J., and Judd, S. J. (2002). The effect of obesity on the ratio of type 3, 17 $\beta$-hydroxysteroid dehydrogenase mRNA to cytochrome $\mathrm{P} 450$ aromatase mRNA in subcutaneous 
abdominal and intra-abdominal adipose tissue of women. Int. J. Obes. Relat. Metab. Disord. 26, 165-175. doi: 10.1038/sj.ijo.0801886

Cornel, K. M., Krakstad, C., Delvoux, B., Xanthoulea, S., Jori, B., Bongers, M. Y., et al. (2017). High mRNA levels of $17 \beta$-hydroxysteroid dehydrogenase type 1 correlate with poor prognosis in endometrial cancer. Mol. Cell. Endocrinol. 442, 51-57. doi: 10.1016/j.mce.2016.11.030

Cornel, K. M., Kruitwagen, R. F., Delvoux, B., Visconti, L., Van de Vijver, K. K., Day, J. M., et al. (2012). Overexpression of $17 \beta$-Hydroxysteroid Dehydrogenase Type 1 Increases the exposure of endometrial cancer to $17 \beta$-Estradiol. J. Clin. Endocrinol. Metab. 97, E591-E601. doi: 10.1210/jc.2011-2994

Cornel, K. M. C., Bongers M. Y., Kruitwagen, R. P. F. M., Romano, A. (2018). Local estrogen metabolism (intracrinology) in endometrial cancer: a systematic review. Mol. Cell. Endocrinol. Accepted for publication.

Couse, J. F., and Korach, K. S. (1999). Estrogen receptor null mice: what have we learned and where will they lead us? Endocr. Rev. 20, 358-417.

Couse, J. F., Lindzey, J., Grandien, K., Gustafsson, J. A., and Korach, K. S. (1997). Tissue distribution and quantitative analysis of estrogen receptor$\alpha(E R \alpha)$ and estrogen receptor- $\beta$ (ER $\beta)$ messenger ribonucleic acid in the wild-type and ER $\alpha$-knockout mouse. Endocrinology 138, 4613-4621. doi: 10.1210/endo.138.11.5496

Couse, J. F., Yates, M. M., Walker, V. R., and Korach, K. S. (2003). Characterization of the hypothalamic-pituitary-gonadal axis in estrogen receptor (ER) Null mice reveals hypergonadism and endocrine sex reversal in females lacking ER $\alpha$ but not ERß. Mol. Endocrinol. 17, 1039-1053. doi: 10.1210/me.2002-0398

Dalla Valle, L., Toffolo, V., Nardi, A., Fiore, C., Armanini, D., Belvedere, P., et al. (2007). The expression of the human steroid sulfatase-encoding gene is driven by alternative first exons. J. Steroid Biochem. Mol. Biol. 107, 22-29. doi: 10.1016/j.jsbmb.2007.05.004

Das, A., Mantena, S. R., Kannan, A., Evans, D. B., Bagchi, M. K., and Bagchi, I. C. (2009). De novo synthesis of estrogen in pregnant uterus is critical for stromal decidualization and angiogenesis. Proc. Natl. Acad. Sci. U.S.A. 106, 12542-12547. doi: 10.1073/pnas.0901647106

Dassen, H., Punyadeera, C., Kamps, R., Delvoux, B., Van Langendonckt, A., Donnez, J., et al. (2007). Estrogen metabolizing enzymes in endometrium and endometriosis. Hum. Reprod. 22, 3148-3158. doi: 10.1093/humrep/dem310

Davies, W., Humby, T., Trent, S., Eddy, J. B., Ojarikre, O. A., and Wilkinson, L. S. (2014). Genetic and pharmacological modulation of the steroid sulfatase axis improves response control; comparison with drugs used in ADHD. Neuropsychopharmacology 39, 2622-2632. doi: 10.1038/npp.2014.115

Day, J. M., Foster, P. A., Tutill, H. J., Schmidlin, F., Sharland, C. M., Hargrave, J. D., et al. (2013). STX2171, a 17 $\beta$-hydroxysteroid dehydrogenase type 3 inhibitor, is efficacious in vivo in a novel hormone-dependent prostate cancer model. Endocr. Relat. Cancer 20, 53-64. doi: 10.1530/ERC-12-0231

De Graaff, A. A., D’Hooghe, T. M., Dunselman, G. A., Dirksen, C. D., Hummelshoj, L., Consortium, W. E., et al. (2013). The significant effect of endometriosis on physical, mental and social wellbeing: results from an international crosssectional survey. Hum. Reprod. 28, 2677-2685. doi: 10.1093/humrep/det284

De Graaff, A. A., Dirksen, C. D., Simoens, S., De Bie, B., Hummelshoj, L., D'Hooghe, T. M., et al. (2015). Quality of life outcomes in women with endometriosis are highly influenced by recruitment strategies. Hum. Reprod. 30, 1331-1341. doi: 10.1093/humrep/dev084

De Graaff, A. A., Van Lankveld, J., Smits, L. J., Van Beek, J. J., and Dunselman, G. A. (2016). Dyspareunia and depressive symptoms are associated with impaired sexual functioning in women with endometriosis, whereas sexual functioning in their male partners is not affected. Hum. Reprod. 31, 2577-2586. doi: 10.1093/humrep/dew215

De Preter, V., Arijs, I., Windey, K., Vanhove, W., Vermeire, S., Schuit, F., et al. (2012). Impaired butyrate oxidation in ulcerative colitis is due to decreased butyrate uptake and a defect in the oxidation pathway. Inflamm. Bowel Dis. 18, 1127-1136. doi: 10.1002/ibd.21894

Delvoux, B., D’Hooghe, T., Kyama, C., Koskimies, P., Hermans, R. J., Dunselman, G. A., et al. (2014). Inhibition of type 1, 17ß-hydroxysteroid dehydrogenase impairs the synthesis of $17 \beta$-estradiol in endometriosis lesions. J. Clin. Endocrinol. Metab. 99, 276-284. doi: 10.1210/jc.2013-2851

Delvoux, B., Groothuis, P., D’Hooghe, T., Kyama, C., Dunselman, G., and Romano, A. (2009). Increased production of $17 \beta$-estradiol in endometriosis lesions is the result of impaired metabolism. J. Clin. Endocrinol. Metab. 94, 876-883. doi: $10.1210 /$ jc. $2008-2218$
Delvoux, B., Husen, B., Aldenhoff, Y., Koole, L., Dunselman, G., Thole, H., et al. (2007). A sensitive HPLC method for the assessment of metabolic conversion of estrogens. J. Steroid Biochem. Mol. Biol. 104, 246-251. doi: 10.1016/j.jsbmb.2007.03.006

Deng, H. Z., You, C., Xing, Y., Chen, K. Y., and Zou, X. B. (2016), A Family-Based Association Study of CYP11A1 and CYP11B1 Gene Polymorphisms With Autism in Chinese Trios. J. Child Neurol. 31, 733-737. doi: $10.1177 / 0883073815620672$

Devroey, P., Bourgain, C., Macklon, N. S., and Fauser, B. C. (2004). Reproductive biology and IVF: ovarian stimulation and endometrial receptivity. Trends Endocrinol. Metab. 15, 84-90. doi: 10.1016/j.tem.2004.01.009

Dheenadayalu, K., Mak, I., Gordts, S., Campo, R., Higham, J., Puttemans, P., et al. (2002). Aromatase P450 messenger RNA expression in eutopic endometrium is not a specific marker for pelvic endometriosis. Fertil. Steril. 78, 825-829. doi: 10.1016/S0015-0282(02)03324-1

Di Costanzo, L., Penning, T. M., and Christianson, D. W. (2009). Aldo-keto reductases in which the conserved catalytic histidine is substituted. Chem. Biol. Interact. 178, 127-133. doi: 10.1016/j.cbi.2008.10.046

Dias, N. J., and Selcer, K. W. (2014). Steroid sulfatase mediated growth Sof human MG-63 pre-osteoblastic cells. Steroids 88, 77-82. doi: 10.1016/j.steroids.2014.07.001

Dias, N. J., and Selcer, K. W. (2016). Steroid sulfatase in the human MG63 preosteoblastic cell line: Antagonistic regulation by glucocorticoids and NFkappaB. Mol. Cell. Endocrinol. 420, 85-96. doi: 10.1016/j.mce.2015.11.029

Dickinson, M. E., Flenniken, A. M., Ji, X., Teboul, L., Wong, M. D., White, J. K., et al. (2016). High-throughput discovery of novel developmental phenotypes. Nature 537, 508-514. doi: 10.1038/nature 19356

Doherty, J. A., Weiss, N. S., Freeman, R. J., Dightman, D. A., Thornton, P. J., Houck, J. R., et al. (2005). Genetic factors in catechol estrogen metabolism in relation to the risk of endometrial cancer. Cancer Epidemiol. Biomarkers Prev. 14, 357-366. doi: 10.1158/1055-9965.EPI-04-0479

Dohi, O., Hatori, M., Suzuki, T., Ono, K., Hosaka, M., Akahira, J., et al. (2008). Sex steroid receptors expression and hormone-induced cell proliferation in human osteosarcoma. Cancer Sci. 99, 518-523. doi: 10.1111/j.1349-7006.2007.00673.x

Dong, Y., Qiu, Q. Q., Debear, J., Lathrop, W. F., Bertolini, D. R., and Tamburini, P. P. (1998). 17ß-hydroxysteroid dehydrogenases in human bone cells. J. Bone Miner. Res. 13, 1539-1546. doi: 10.1359/jbmr.1998.13.10.1539

Driessen, C. A., Winkens, H. J., Hoffmann, K., Kuhlmann, L. D., Janssen, B. P., Van Vugt, A. H., et al. (2000). Disruption of the 11-cis-retinol dehydrogenase gene leads to accumulation of cis-retinols and cis-retinyl esters. Mol. Cell. Biol. 20, 4275-4287. doi: 10.1128/MCB.20.12.4275-4287.2000

Drzewiecka, H., Galecki, B., Jarmolowska-Jurczyszyn, D., Kluk, A., Dyszkiewicz, W., and Jagodzinski, P. P. (2015). Increased expression of 17-betahydroxysteroid dehydrogenase type 1 in non-small cell lung cancer. Lung Cancer 87, 107-116. doi: 10.1016/j.lungcan.2014.12.012

Drzewiecka, H., and Jagodzinski, P. P. (2012). Conversion of estrone to 17beta-estradiol in human non-small-cell lung cancer cells in vitro. Biomed. Pharmacother. 66, 530-534. doi: 10.1016/j.biopha.2012.02.006

Duell, E. J., Travier, N., Lujan-Barroso, L., Boutron-Ruault, M. C., ClavelChapelon, F., Palli, D., et al. (2010). Menstrual and reproductive factors, exogenous hormone use, and gastric cancer risk in a cohort of women from the European Prospective Investigation Into Cancer and Nutrition. Am. J. Epidemiol. 172, 1384-1393. doi: 10.1093/aje/ kwq321

Dunselman, G. A., Vermeulen, N., Becker, C., Calhaz-Jorge, C., D'Hooghe, T., De Bie, B., et al. (2014). ESHRE guideline: management of women with endometriosis. Hum. Reprod. 29, 400-412. doi: 10.1093/humrep/det457

Ellem, S. J., Wang, H., Poutanen, M., and Risbridger, G. P. (2009). Increased endogenous estrogen synthesis leads to the sequential induction of prostatic inflammation (prostatitis) and prostatic pre-malignancy. Am. J. Pathol. 175, 1187-1199. doi: 10.2353/ajpath.2009.081107

Endo, S., Miyagi, N., Matsunaga, T., Hara, A., and Ikari, A. (2016). Human dehydrogenase/reductase (SDR family) member 11 is a novel type of $17 \beta$ hydroxysteroid dehydrogenase. Biochem. Biophys. Res. Commun. 472, 231-236. doi: 10.1016/j.bbrc.2016.01.190

English, M. A., Hughes, S. V., Kane, K. F., Langman, M. J., Stewart, P. M., and Hewison, M. (2000). Oestrogen inactivation in the colon: analysis of the expression and regulation of $17 \beta$-hydroxysteroid dehydrogenase 
isozymes in normal colon and colonic cancer. Br. J. Cancer 83, 550-558. doi: 10.1054/bjoc.2000.1324

English, M. A., Kane, K. F., Cruickshank, N., Langman, M. J., Stewart, P. M., and Hewison, M. (1999). Loss of estrogen inactivation in colonic cancer. J. Clin. Endocrinol. Metab. 84, 2080-2085. doi: 10.1210/jcem.84.6.5772

Enríquez, J., Larrea, F., Santillan, R., Hernandez, A., Herrero, B., PerezPalacios, G., et al. (2013). Neonatal rat osteoblasts bioconvert testosterone to non-phenolic metabolites with estrogen-like effects on bone cell proliferation and differentiation. Horm. Mol. Biol. Clin. Investig. 13, 41-49. doi: 10.1515/hmbci-2012-0035

Falk, R. T., Dallal, C. M., Lacey, J. V. Jr., Bauer, D. C., Buist, D. S., Cauley, J. A., et al. (2015). Estrogen metabolites are not associated with colorectal cancer risk in postmenopausal women. Cancer Epidemiol. Biomarkers Prev. 24, 1419-1422. doi: 10.1158/1055-9965.EPI-15-0541

Ferrero, S., Gillott, D. J., Venturini, P. L., and Remorgida, V. (2011). Use of aromatase inhibitors to treat endometriosis-related pain symptoms: a systematic review. Reprod. Biol. Endocrinol. 9, 89. doi: 10.1186/1477-7827-9-89

Fisher, C. R., Graves, K. H., Parlow, A. F., and Simpson, E. R. (1998). Characterization of mice deficient in aromatase (ArKO) because of targeted disruption of the cyp19 gene. Proc. Natl. Acad. Sci. U.S.A. 95, 6965-6970. doi: 10.1073/pnas.95.12.6965

Flach, K. D., and Zwart, W. (2016). The first decade of estrogen receptor cistromics in breast cancer. J. Endocrinol. 229 R43-56. doi: 10.1530/JOE-16-0003

Fontein, D. B., Houtsma, D., Nortier, J. W., Baak-Pablo, R. F., Kranenbarg, E. M., van der Straaten, T. R., et al. (2014). Germline variants in the $\mathrm{CYP}_{19} \mathrm{~A}_{1}$ gene are related to specific adverse events in aromatase inhibitor users: a substudy of Dutch patients in the TEAM trial. Breast Cancer Res. Treat. 144, 599-606. doi: 10.1007/s10549-014-2873-2

Foster, P. A. (2013). Oestrogen and colorectal cancer: mechanisms and controversies. Int. J. Colorectal Dis. 28, 737-749. doi: 10.1007/s00384-012-1628-y

Foster, P. A., Reed, M. J., and Purohit, A. (2008a). Recent developments of steroid sulfatase inhibitors as anti-cancer agents. Anticancer. Agents Med. Chem. 8, 732-738. doi: 10.2174/187152008785914815

Foster, P. A., Woo, L. W., Potter, B. V., Reed, M. J., and Purohit, A. (2008b). The use of steroid sulfatase inhibitors as a novel therapeutic strategy against hormone-dependent endometrial cancer. Endocrinology 149, 4035-4042. doi: 10.1210/en.2008-0223

Fouad Mansour, M., Pelletier, M., Boulet, M. M., Mayrand, D., Brochu, G., Lebel, S., et al. (2015). Oxidative activity of $17 \beta$-hydroxysteroid dehydrogenase on testosterone in male abdominal adipose tissues and cellular localization of 17ß-HSD type 2. Mol. Cell. Endocrinol. 414, 168-176. doi: 10.1016/j.mce.2015.06.016

Freedman, N. D., Ahn, J., Hou, L., Lissowska, J., Zatonski, W., Yeager, M., et al. (2009). Polymorphisms in estrogen- and androgen-metabolizing genes and the risk of gastric cancer. Carcinogenesis 30, 71-77. doi: 10.1093/carcin/bgn258

Freedman, N. D., Chow, W. H., Gao, Y. T., Shu, X. O., Ji, B. T., Yang, G., et al. (2007). Menstrual and reproductive factors and gastric cancer risk in a large prospective study of women. Gut 56, 1671-1677. doi: 10.1136/gut.2007.129411

Fritsch, M., Orfanos, C. E., and Zouboulis, C. C. (2001). Sebocytes are the key regulators of androgen homeostasis in human skin. J. Invest. Dermatol. 116, 793-800. doi: 10.1046/j.1523-1747.2001.01312.x

Frycz, B. A., Murawa, D., Borejsza-Wysocki, M., Marciniak, R., Murawa, P., Drews, M., et al. (2015). Expression of 17 $\beta$-hydroxysteroid dehydrogenase type 2 is associated with some clinicopathological features in gastric cancer. Biomed. Pharmacother. 70, 24-27. doi: 10.1016/j.biopha.2014.12.042

Frycz, B. A., Murawa, D., Borejsza-Wysocki, M., Wichtowski, M., Spychala, A., Marciniak, R., et al. (2016). Transcript level of AKR1C3 is down-regulated in gastric cancer. Biochem. Cell Biol. 94, 138-146. doi: 10.1139/bcb-201 5-0096

Fujikawa, H., Okura, F., Kuwano, Y., Sekizawa, A., Chiba, H., Shimodaira, K., et al. (1997). Steroid sulfatase activity in osteoblast cells. Biochem. Biophys. Res. Commun. 231, 42-47. doi: 10.1006/bbrc.1996.6038

Gangloff, A., Shi, R., Nahoum, V., and Lin, S. X. (2003). Pseudo-symmetry of C19 steroids, alternative binding orientations, and multispecificity in human estrogenic 17 $\beta$-hydroxysteroid dehydrogenase. FASEB J. 17, 274-276. doi: 10.1096/fj.02-0397fje
Gao, J., He, J., Shi, X., Stefanovic-Racic, M., Xu, M., O’Doherty, R. M., et al. (2012). Sex-specific effect of estrogen sulfotransferase on mouse models of type 2 diabetes. Diabetes 61, 1543-1551. doi: 10.2337/db11-1152

Gargano, E. M., Allegretta, G., Perspicace, E., Carotti, A., Van Koppen, C., Frotscher, M., et al. (2015). 17 $\beta$-Hydroxysteroid dehydrogenase Type 2 inhibition: discovery of selective and metabolically stable compounds inhibiting both the human enzyme and its murine ortholog. PLoS ONE 10:e0134754. doi: 10.1371/journal.pone.0134754

Ghosh, D., Dhara, S., Kumar, A., and Sengupta, J. (1999). Immunohistochemical localization of receptors for progesterone and oestradiol-17 beta in the implantation site of the rhesus monkey. Hum. Reprod. 14, 505-514. doi: 10.1093/humrep/14.2.505

Gibbs, T. T., Russek, S. J., and Farb, D. H. (2006). Sulfated steroids as endogenous neuromodulators. Pharmacol. Biochem. Behav. 84, 555-567. doi: 10.1016/j.pbb.2006.07.031

Gibson, D. A., McInnes, K. J., Critchley, H. O., and Saunders, P. T. (2013). Endometrial Intracrinology-generation of an estrogen-dominated microenvironment in the secretory phase of women. J. Clin. Endocrinol. Metab. 98, E1802-E1806. doi: 10.1210/jc.2013-2140

Gilligan, L. C., Gondal, A., Tang, V., Hussain, M. T., Arvaniti, A., Hewitt, A. M., et al. (2017). Estrone sulfate transport and steroid sulfatase activity in colorectal cancer: implications for hormone replacement therapy. Front. Pharmacol. 8:103. doi: 10.3389/fphar.2017.00103

González-Arenas, A., and Agramonte-Hevia, J. (2012). Sex steroid hormone effects in normal and pathologic conditions in lung physiology. Mini Rev. Med. Chem. 12, 1055-1062. doi: 10.2174/138955712802762194

Goodarzi, M. O., Antoine, H. J., and Azziz, R. (2007). Genes for enzymes regulating dehydroepiandrosterone sulfonation are associated with levels of dehydroepiandrosterone sulfate in polycystic ovary syndrome. J. Clin. Endocrinol. Metab. 92, 2659-2664. doi: 10.1210/jc.2006-2600

Goodarzi, M. O., Shah, N. A., Antoine, H. J., Pall, M., Guo, X., and Azziz, R. (2006). Variants in the $5 \alpha$-reductase type 1 and type 2 genes are associated with polycystic ovary syndrome and the severity of hirsutism in affected women. $J$. Clin. Endocrinol. Metab. 91, 4085-4091. doi: 10.1210/jc.2006-0227

Groothuis, P. G., Dassen, H. H., Romano, A., and Punyadeera, C. (2007). Estrogen and the endometrium: lessons learned from gene expression profiling in rodents and human. Hum. Reprod. Update 13, 405-417. doi: 10.1093/humupd/dmm009

Gruber, C. J., Tschugguel, W., Schneeberger, C., and Huber, J. C. (2002). Production and actions of estrogens. N. Engl. J. Med. 346, 340-352. doi: 10.1056/NEJMra000471

Guillot, F., Garcia, A., Salou, M., Brouard, S., Laplaud, D. A., and Nicot, A. B. (2015). Transcript analysis of laser capture microdissected white matter astrocytes and higher phenol sulfotransferase 1A1 expression during autoimmune neuroinflammation. J. Neuroinflammation 12, 130. doi: 10.1186/s12974-015-0348-y

Gulyaeva, L. F., Mikhailova, O. N., PustyInyak, V. O., Kim, I. V. IV., Gerasimov, A. V., Krasilnikov, S. E., et al. (2008). Comparative analysis of SNP in estrogen-metabolizing enzymes for ovarian, endometrial, and breast cancers in Novosibirsk, Russia. Adv. Exp. Med. Biol. 617, 359-366. doi: 10.1007/978-0-387-69080-3_34

Gylfe, A. E., Katainen, R., Kondelin, J., Tanskanen, T., Cajuso, T., Hanninen, U., et al. (2013). Eleven candidate susceptibility genes for common familial colorectal cancer. PLoS Genet. 9:e1003876. doi: 10.1371/journal.pgen.1003876

Hakkarainen, J., Jokela, H., Pakarinen, P., Heikela, H., Katkanaho, L., Vandenput, L., et al. (2015). Hydroxysteroid (17 $\beta$ )-dehydrogenase 1-deficient female mice present with normal puberty onset but are severely subfertile due to a defect in luteinization and progesterone production. FASEB J. 29, 3806-3816. doi: 10.1096/fj.14-269035

He, D., Frost, A. R., and Falany, C. N. (2005a). Identification and immunohistochemical localization of Sulfotransferase 2B1b (SULT2B1b) in human lung. Biochim Biophys Act. 1724, 119-126. doi: 10.1016/j.bbagen.2005.03.018

He, D., Meloche, C. A., Dumas, N. A., Frost, A. R., and Falany, C. N. (2004). Different subcellular localization of sulphotransferase 2B1b in human placenta and prostate. Biochem. J. 379, 533-540. doi: 10.1042/bj20 031524 
He, W., Gauri, M., Li, T., Wang, R., and Lin, S. X. (2016). Current knowledge of the multifunctional $17 \beta$-hydroxysteroid dehydrogenase type 1 (HSD17B1). Gene 588, 54-61. doi: 10.1016/j.gene.2016.04.031

He, X. Y., Wegiel, J., Yang, Y. Z., Pullarkat, R., Schulz, H., and Yang, S. Y. (2005b). Type 10, 17ß-hydroxysteroid dehydrogenase catalyzing the oxidation of steroid modulators of gamma-aminobutyric acid type A receptors. Mol. Cell. Endocrinol. 229, 111-117. doi: 10.1016/j.mce.2004.08.011

He, X. Y., and Yang, S. Y. (2006). Roles of type 10, 17ß-hydroxysteroid dehydrogenase in intracrinology and metabolism of isoleucine and fatty acids. Endocr. Metab. Immune Disord. Drug Targets 6, 95-102. doi: $10.2174 / 187153006776056639$

Heijmans, J., Wielenga, M. C., Rosekrans, S. L., van Lidth de Jeude, J. F., Roelofs, J., Groothuis, P., et al. (2014). Oestrogens promote tumorigenesis in a mouse model for colitis-associated cancer. Gut 63, 310-316. doi: 10.1136/gutjnl-2012-304216

Her, C., Szumlanski, C., Aksoy, I. A., and Weinshilboum, R. M. (1996). Human jejunal estrogen sulfotransferase and dehydroepiandrosterone sulfotransferase: immunochemical characterization of individual variation. Drug Metab. Dispos. 24, 1328-1335.

Hevir, N., Ribic-Pucelj, M., and Lanisnik Rizner, T. (2013). Disturbed balance between phase I and II metabolizing enzymes in ovarian endometriosis: a source of excessive hydroxy-estrogens and ROS? Mol. Cell. Endocrinol. 367, 74-84. doi: 10.1016/j.mce.2012.12.019

Hevir, N., Sinkovec, J., and Rizner, T. L. (2011a). Disturbed expression of phase I and phase II estrogen-metabolizing enzymes in endometrial cancer: lower levels of CYP1B1 and increased expression of S-COMT. Mol. Cell. Endocrinol. 331, 158-167. doi: 10.1016/j.mce.2010.09.011

Hevir, N., Vouk, K., Sinkovec, J., Ribic-Pucelj, M., and Rizner, T. L. (2011b). Aldoketo reductases AKR1C1, AKR1C2 and AKR1C3 may enhance progesterone metabolism in ovarian endometriosis. Chem. Biol. Interact. 191, 217-226. doi: 10.1016/j.cbi.2011.01.003

Hewitt, S. C., Winuthayanon, W., and Korach, K. S. (2016). What's new in estrogen receptor action in the female reproductive tract. J. Mol. Endocrinol. 56 R55-71. doi: 10.1530/JME-15-0254

Hilborn, E., Stal, O., and Jansson, A. (2017). Estrogen and androgen-converting enzymes $17 \beta$-hydroxysteroid dehydrogenase and their involvement in cancer: with a special focus on $17 \beta$-hydroxysteroid dehydrogenase type 1,2 , and breast cancer. Oncotarget 8, 30552-30562. doi: 10.18632/oncotarget.15547

Hogan, A. M., Collins, D., Baird, A. W., and Winter, D. C. (2009). Estrogen and gastrointestinal malignancy. Mol. Cell. Endocrinol. 307, 19-24. doi: 10.1016/j.mce.2009.03.016

Honda, S., Harada, N., Ito, S., Takagi, Y., and Maeda, S. (1998). Disruption of sexual behavior in male aromatase-deficient mice lacking exons 1 and 2 of the cyp19 gene. Biochem. Biophys. Res. Commun. 252, 445-449. doi: 10.1006/bbrc. 1998.9672

Hovorkova, P., Kristofikova, Z., Horinek, A., Ripova, D., Majer, E., Zach, P., et al. (2008). Lateralization of $17 \beta$-hydroxysteroid dehydrogenase type 10 in hippocampi of demented and psychotic people. Dement. Geriatr. Cogn. Disord. 26, 193-198. doi: 10.1159/000151778

Hu, M. C., Hsu, N. C., El Hadj, N. B., Pai, C. I., Chu, H. P., Wang, C. K., et al. (2002). Steroid deficiency syndromes in mice with targeted disruption of Cyp11a1. Mol. Endocrinol. 16, 1943-1950. doi: 10.1210/me.2002-0055

Huang, C. C., Shih, M. C., Hsu, N. C., Chien, Y., and Chung, B. C. (2012). Fetal glucocorticoid synthesis is required for development of fetal adrenal medulla and hypothalamus feedback suppression. Endocrinology 153, 4749-4756. doi: 10.1210/en.2012-1258

Huang, X. F., and Luu-The, V. (2000). Molecular characterization of a first human 3( $\alpha->\beta)$-hydroxysteroid epimerase. J. Biol. Chem. 275, 29452-29457. doi: 10.1074/jbc.M000562200

Huber, A., Keck, C. C., Hefler, L. A., Schneeberger, C., Huber, J. C., Bentz, E. K., et al. (2005). Ten estrogen-related polymorphisms and endometriosis: a study of multiple gene-gene interactions. Obstet. Gynecol. 106, 1025-1031. doi: 10.1097/01.AOG.0000185259.01648.41

Hudelist, G., Czerwenka, K., Keckstein, J., Haas, C., Fink-Retter, A., Gschwantler-Kaulich, D., et al. (2007). Expression of aromatase and estrogen sulfotransferase in eutopic and ectopic endometrium: evidence for unbalanced estradiol production in endometriosis. Reprod. Sci. 14, 798-805. doi: 10.1177/1933719107309120
Hughes, S. V., Robinson, E., Bland, R., Lewis, H. M., Stewart, P. M., and Hewison, M. (1997). 1,25-dihydroxyvitamin D3 regulates estrogen metabolism in cultured keratinocytes. Endocrinology 138, 3711-3718. doi: 10.1210/endo.138.9.5406

Huhtinen, K., Desai, R., Stahle, M., Salminen, A., Handelsman, D. J., Perheentupa, A., et al. (2012a). Endometrial and endometriotic concentrations of estrone and estradiol are determined by local metabolism rather than circulating levels. $J$. Clin. Endocrinol. Metab. 97, 4228-4235. doi: 10.1210/jc.2012-1154

Huhtinen, K., Saloniemi-Heinonen, T., Keski-Rahkonen, P., Desai, R., Laajala, D., Stahle, M., et al. (2014). Intra-tissue steroid profiling indicates differential progesterone and testosterone metabolism in the endometrium and endometriosis lesions. J. Clin. Endocrinol. Metab. 99 E2188-2197. doi: 10.1210/jc.2014-1913

Huhtinen, K., Stahle, M., Perheentupa, A., and Poutanen, M. (2012b). Estrogen biosynthesis and signaling in endometriosis. Mol. Cell. Endocrinol. 358, 146-154. doi: 10.1016/j.mce.2011.08.022

Husen, B., Huhtinen, K., Poutanen, M., Kangas, L., Messinger, J., and Thole, H. (2006). Evaluation of inhibitors for 17ß-hydroxysteroid dehydrogenase type 1 in vivo in immunodeficient mice inoculated with MCF-7 cells stably expressing the recombinant human enzyme. Mol. Cell. Endocrinol. 248, 109-113. doi: 10.1016/j.mce.2005.11.042

Ihlenfeldt, W. D., Bolton, E. E., and Bryant, S. H. (2009). The PubChem chemical structure sketcher. J. Cheminform. 1:20. doi: 10.1186/1758-2946-1-20

Iida, S., Kakinuma, H., Miki, Y., Abe, K., Sakurai, M., Suzuki, S., et al. (2013). Steroid sulphatase and oestrogen sulphotransferase in human non-small-cell lung carcinoma. Br. J. Cancer 108, 1415-1424. doi: 10.1038/bjc.2013.84

Ikeda, K., Shiraishi, K., Yoshida, A., Shinchi, Y., Sanada, M., Motooka, Y., et al. (2016). Synchronous multiple lung adenocarcinomas: estrogen concentration in peripheral lung. PLOS ONE 11:e0160910. doi: 10.1371/journal.pone.0160910

Iqbal, M. J., Greenway, B., Wilkinson, M. L., Johnson, P. J., and Williams, R. (1983). Sex-steroid enzymes, aromatase and 5 alpha-reductase in the pancreas: a comparison of normal adult, foetal and malignant tissue. Clin. Sci. 65, 71-75. doi: $10.1042 / \mathrm{cs} 0650071$

Ishida, M., Choi, J. H., Hirabayashi, K., Matsuwaki, T., Suzuki, M., Yamanouchi, K., et al. (2007). Reproductive phenotypes in mice with targeted disruption of the 20a-hydroxysteroid dehydrogenase gene. J. Reprod. Dev. 53, 499-508. doi: $10.1262 /$ jrd. 18125

Ito, K., Miki, Y., Suzuki, T., McNamara, K. M., and Sasano, H. (2016). In situ androgen and estrogen biosynthesis in endometrial cancer: focus on androgen actions and intratumoral production. Endocr. Relat. Cancer 23 R323-335. doi: 10.1530/ERC-15-0470

Ito, K., Suzuki, T., Akahira, J., Moriya, T., Kaneko, C., Utsunomiya, H., et al. (2002). Expression of androgen receptor and $5 \alpha$-reductases in the human normal endometrium and its disorders. Int. J. Cancer 99, 652-657. doi: $10.1002 / \mathrm{ijc} .10394$

Ito, K., Utsunomiya, H., Suzuki, T., Saitou, S., Akahira, J., Okamura, K., et al. (2006). 17ß-hydroxysteroid dehydrogenases in human endometrium and its disorders. Mol. Cell. Endocrinol. 248, 136-140. doi: 10.1016/j.mce.2005.11.038

Iwasa, A., Arakaki, R., Honma, N., Ushio, A., Yamada, A., Kondo, T., et al. (2015). Aromatase controls Sjogren syndrome-like lesions through monocyte chemotactic protein-1 in target organ and adipose tissue-associated macrophages. Am. J. Pathol. 185, 151-161. doi: 10.1016/j.ajpath.2014. 09.006

Jakob, F., Siggelkow, H., Homann, D., Kohrle, J., Adamski, J., and Schutze, N. (1997). Local estradiol metabolism in osteoblast- and osteoclast-like cells. J. Steroid Biochem. Mol. Biol. 61, 167-174. doi: 10.1016/S0960-0760(97)80009-X

Janssen, J. M., Bland, R., Hewison, M., Coughtrie, M. W., Sharp, S., Arts, J., et al. (1999). Estradiol formation by human osteoblasts via multiple pathways: relation with osteoblast function. J. Cell. Biochem. 75, 528-537. doi: 10.1002/ (SICI) 1097-4644(19991201)75:3\&lt;528::AID-JCB16\&gt;3.0.CO;2-3

Jäntti, S. E., Tammimaki, A., Raattamaa, H., Piepponen, P., Kostiainen, R., and Ketola, R. A. (2010). Determination of steroids and their intact glucuronide conjugates in mouse brain by capillary liquid chromatography-tandem mass spectrometry. Anal. Chem. 82, 3168-3175. doi: 10.1021/ac902321z

Järvensivu, P., Heinosalo, T., Hakkarainen, J., Kronqvist, P., Saarinen, N., and Poutanen, M. (2018). HSD17B1 expression induces inflammation-aided rupture of mammary gland myoepithelium. Endocr. Relat. Cancer 25, 393-406. doi: 10.1530/ERC-17-0476 
Järvensivu, P., Saloniemi-Heinonen, T., Awosanya, M., Koskimies, P., Saarinen, N., and Poutanen, M. (2015). HSD17B1 expression enhances estrogen signaling stimulated by the low active estrone, evidenced by an estrogen responsive element-driven reporter gene in vivo. Chem. Biol. Interact. 234, 126-134. doi: 10.1016/j.cbi.2015.01.008

Jeon, Y. T., Park, S. Y., Kim, Y. B., Kim, J. W., Park, N. H., Kang, S. B., et al. (2007). Aromatase expression was not detected by immunohistochemistry in endometrial cancer. Ann. N. Y. Acad. Sci. 1095, 70-75. doi: 10.1196/annals.1397.010

Jin, Y., Mesaros, A. C., Blair, I. A., and Penning, T. M. (2011). Stereospecific reduction of $5 \beta$-reduced steroids by human ketosteroid reductases of the AKR (aldo-keto reductase) superfamily: role of AKR1C1-AKR1C4 in the metabolism of testosterone and progesterone via the $5 \beta$-reductase pathway. Biochem. J. 437, 53-61. doi: 10.1042/BJ20101804

Jokela, H., Rantakari, P., Lamminen, T., Strauss, L., Ola, R., Mutka, A. L., et al. (2010). Hydroxysteroid (17 $\beta$ ) dehydrogenase 7 activity is essential for fetal de novo cholesterol synthesis and for neuroectodermal survival and cardiovascular differentiation in early mouse embryos. Endocrinology 151, 1884-1892. doi: 10.1210/en.2009-0928

Jones, A. L., Hume, R., Bamforth, K. J., and Coughtrie, M. W. (1992). Estrogen and phenol sulfotransferase activities in human fetal lung. Early Hum. Dev. 28, 65-77. doi: 10.1016/0378-3782(92)90007-4

Jones, M. R., Mathur, R., Cui, J., Guo, X., Azziz, R., and Goodarzi, M. O. (2009). Independent confirmation of association between metabolic phenotypes of polycystic ovary syndrome and variation in the type $6,17 \beta$ hydroxysteroid dehydrogenase gene. J. Clin. Endocrinol. Metab. 94, 5034-5038. doi: $10.1210 /$ jc.2009-0931

Jongen, V. H., Thijssen, J. H., Hollema, H., Donker, G. H., Santema, J. G., Van der Zee, A. G., et al. (2005). Is aromatase cytochrome P450 involved in the pathogenesis of endometrioid endometrial cancer? Int. J. Gynecol. Cancer 15, 529-536. doi: 10.1111/j.1525-1438.2005.15320.x

Kancheva, L., Hill, M., Vcelakova, H., Vrbikova, J., Pelikanova, T., and Starka, L. (2007). The identification and simultaneous quantification of neuroactive androstane steroids and their polar conjugates in the serum of adult men, using gas chromatography-mass spectrometry. Steroids 72, 792-801. doi: 10.1016/j.steroids.2007.06.006

Kassem, M., Okazaki, R., Harris, S. A., Spelsberg, T. C., Conover, C. A., and Riggs, B. L. (1998). Estrogen effects on insulin-like growth factor gene expression in a human osteoblastic cell line with high levels of estrogen receptor. Calcif. Tissue Int. 62, 60-66. doi: 10.1007/s002239900395

Kemilainen, H., Adam, M., Maki-Jouppila, J., Damdimopoulou, P., Damdimopoulos, A. E., Kere, J., et al. (2016). The hydroxysteroid (17 $\beta$ ) dehydrogenase family gene HSD17B12 Is involved in the prostaglandin synthesis pathway, the ovarian function, and regulation of fertility. Endocrinology 157, 3719-3730. doi: 10.1210/en.2016-1252

Kennelly, R., Kavanagh, D. O., Hogan, A. M., and Winter, D. C. (2008). Oestrogen and the colon: potential mechanisms for cancer prevention. Lancet Oncol. 9, 385-391. doi: 10.1016/S1470-2045(08)70100-1

Kikuchi, A., Furutani, T., Azami, H., Watanabe, K., Niimi, T., Kamiyama, Y., et al. (2014). In vitro and in vivo characterisation of ASP9521: a novel, selective, orally bioavailable inhibitor of $17 \beta$-hydroxysteroid dehydrogenase type 5 (17 $\beta$ HSD5; AKR1C3). Invest. New Drugs 32, 860-870. doi: 10.1007/s10637-014-0130-5

Kim, J. H., Cho, H. T., and Kim, Y. J. (2014). The role of estrogen in adipose tissue metabolism: insights into glucose homeostasis regulation. Endocr. J. 61, 1055-1067. doi: 10.1507/endocrj.EJ14-0262

Kim, S., Thiessen, P. A., Bolton, E. E., Chen, J., Fu, G., Gindulyte, A., et al. (2016). PubChem substance and compound databases. Nucleic Acids Res. 44, D1202-D1213. doi: 10.1093/nar/gkv951

Kim, T. S., Maeda, A., Maeda, T., Heinlein, C., Kedishvili, N., Palczewski, K., et al. (2005). Delayed dark adaptation in 11-cis-retinol dehydrogenase-deficient mice: a role of RDH11 in visual processes in vivo. J. Biol. Chem. 280, 8694-8704. doi: 10.1074/jbc.M413172200

Kimoto, T., Ishii, H., Higo, S., Hojo, Y., and Kawato, S. (2010). Semicomprehensive analysis of the postnatal age-related changes in the mRNA expression of sex steroidogenic enzymes and sex steroid receptors in the male rat hippocampus. Endocrinology 151, 5795-5806. doi: 10.1210/en.2010-0581

Kimura, Y., Suzuki, T., Kaneko, C., Darnel, A. D., Akahira, J., Ebina, M., et al. (2003). Expression of androgen receptor and $5 \alpha$-reductase types 1 and 2 in early gestation fetal lung: a possible correlation with branching morphogenesis. Clin. Sci. 105, 709-713. doi: 10.1042/CS20030236

Kitaoka, Y., Kitawaki, J., Koshiba, H., Inoue, S., Ishihara, H., Teramoto, M., et al. (2004). Aromatase cytochrome P450 and estrogen and progesterone receptors in uterine sarcomas: correlation with clinical parameters. J. Steroid Biochem. Mol. Biol. 88, 183-189. doi: 10.1016/j.jsbmb.2003.11.013

Kitawaki, J., Kado, N., Ishihara, H., Koshiba, H., Kitaoka, Y., and Honjo, H. (2002). Endometriosis: the pathophysiology as an estrogen-dependent disease. J. Steroid Biochem. Mol. Biol. 83, 149-155. doi: 10.1016/S0960-0760(02)00260-1

Kitawaki, J., Koshiba, H., Ishihara, H., Kusuki, I., Tsukamoto, K., and Honjo, H. (2000). Progesterone induction of $17 \beta$-hydroxysteroid dehydrogenase type 2 during the secretory phase occurs in the endometrium of estrogen-dependent benign diseases but not in normal endometrium. J. Clin. Endocrinol. Metab. 85, 3292-3296. doi: 10.1210/jcem.85.9.6829

Kitawaki, J., Kusuki, I., Koshiba, H., Tsukamoto, K., Fushiki, S., and Honjo, H. (1999). Detection of aromatase cytochrome P-450 in endometrial biopsy specimens as a diagnostic test for endometriosis. Fertil. Steril. 72, 1100-1106. doi: 10.1016/S0015-0282(99)00424-0

Knapp, P., Chabowski, A., Blachnio-Zabielska, A., Walentowicz-Sadlecka, M., Grabiec, M., and Knapp, P. A. (2013). Expression of estrogen receptors (alpha, beta), cyclooxygenase-2 and aromatase in normal endometrium and endometrioid cancer of uterus. Adv. Med. Sci. 58, 96-103. doi: 10.2478/v10039-012-0055-1

Kobayashi, H., Yoshida, S., Sun, Y. J., Shirasawa, N., and Naito, A. (2013). Postnatal development of gastric aromatase and portal venous estradiol-17 $\beta$ levels in male rats. J. Endocrinol. 218, 117-124. doi: 10.1530/JOE-13-0074

Kohno, M., Okamoto, T., Suda, K., Shimokawa, M., Kitahara, H., Shimamatsu, S., et al. (2014). Prognostic and therapeutic implications of aromatase expression in lung adenocarcinomas with EGFR mutations. Clin. Cancer Res. 20, 3613-3622. doi: 10.1158/1078-0432.CCR-13-2683

Koizumi, M., Momoeda, M., Hiroi, H., Hosokawa, Y., Tsutsumi, R., Osuga, Y., et al. (2010). Expression and regulation of cholesterol sulfotransferase (SULT2B1b) in human endometrium. Fertil. Steril. 93, 1538-1544. doi: 10.1016/j.fertnstert.2009.01.075

Konings, G. F., Cornel, K. M., Xanthoulea, S., Delvoux, B., Skowron, M. A., Kooreman, L., et al. (2018). Blocking 17 $\beta$-hydroxysteroid dehydrogenase type 1 in endometrial cancer: a potential novel endocrine therapeutic approach. $J$. Pathol. 244, 203-214. doi: 10.1002/path.5004

Konings, G. F., Reynaert, N. L., Delvoux, B., Verhamme, F. M., Bracke, K. R., Brusselle, G. G., et al. (2017). Increased levels of enzymes involved in local estradiol synthesis in chronic obstructive pulmonary disease. Mol. Cell. Endocrinol. 443, 23-31. doi: 10.1016/j.mce.2016.12.001

Kreitmann, O., Kreitmann-Gimbal, B., Bayard, F., and Hodgen, G. D. (1979). 17 beta-hydroxysteroid dehydrogenase in monkey endometrium: characterization of enzyme activity, and effects of estradiol alone or in combination with progesterone. Steroids 34, 693-703. doi: 10.1016/0039-128X(79)90139-9

Kríz, L., Bicikova, M., and Hampl, R. (2008a). Roles of steroid sulfatase in brain and other tissues. Physiol. Res. 57, 657-668.

Kríz, L., Bicikova, M., Mohapl, M., Hill, M., Cerny, I., and Hampl, R. (2008b). Steroid sulfatase and sulfuryl transferase activities in human brain tumors. J. Steroid Biochem. Mol. Biol. 109, 31-39. doi: 10.1016/j.jsbmb.2007. 12.004

Laatikainen, T., Laitinen, E. A., and Vihko, R. (1971). Secretion of free and sulfate-conjugated neutral steroids by the human testis. Effect of administration of human chorionic gonadotropin. J. Clin. Endocrinol. Metab. 32, 59-64. doi: 10.1210/jcem-32-1-59

Labrie, F. (1991). Intracrinology. Mol. Cell. Endocrinol. 78, C113-C118. doi: 10.1016/0303-7207(91)90116-A

Labrie, F. (2015). All sex steroids are made intracellularly in peripheral tissues by the mechanisms of intracrinology after menopause. J. Steroid Biochem. Mol. Biol. 145, 133-138. doi: 10.1016/j.jsbmb.2014.06.001

Labrie, F., and Labrie, C. (2013). DHEA and intracrinology at menopause, a positive choice for evolution of the human species. Climacteric 16, 205-213. doi: 10.3109/13697137.2012.733983

Lane, G. (1990). The histochemistry of isocitric and oestradiol-17 beta dehydrogenases in the endometrium of postmenopausal women treated with oestrogens and progestogens. Histochem. J. 22, 45-50. doi: 10.1007/BF019 62878 
Laplante, Y., Rancourt, C., and Poirier, D. (2009). Relative involvement of three $17 \beta$-hydroxysteroid dehydrogenases (types 1, 7 and 12) in the formation of estradiol in various breast cancer cell lines using selective inhibitors. Mol. Cell. Endocrinol. 301, 146-153. doi: 10.1016/j.mce.2008.08.026

Lavasani, S., Chlebowski, R. T., Prentice, R. L., Kato, I., Wactawski-Wende, J., Johnson, K. C., et al. (2015). Estrogen and colorectal cancer incidence and mortality. Cancer 121, 3261-3271. doi: 10.1002/cncr.29464

Lee, D. K., Kurihara, I., Jeong, J. W., Lydon, J. P., DeMayo, F. J., Tsai, M. J., et al. (2010). Suppression of ER $\alpha$ activity by COUP-TFII is essential for successful implantation and decidualization. Mol. Endocrinol. 24, 930-940. doi: 10.1210/me.2009-0531

Lépine, J., Audet-Walsh, E., Gregoire, J., Tetu, B., Plante, M., Menard, V., et al. (2010). Circulating estrogens in endometrial cancer cases and their relationship with tissular expression of key estrogen biosynthesis and metabolic pathways. J. Clin. Endocrinol. Metab. 95, 2689-2698. doi: 10.1210/jc.2010-2648

Li, C., Chen, P., Palladino, A., Narayan, S., Russell, L. K., Sayed, S., et al. (2010). Mechanism of hyperinsulinism in short-chain 3-hydroxyacyl-CoA dehydrogenase deficiency involves activation of glutamate dehydrogenase. J. Biol. Chem. 285, 31806-31818. doi: 10.1074/jbc.M110.123638

Li, Y., Qin, L., Xiao, Z. J., Wang, Y. L., Herva, R., Leng, J. H., et al. (2003). Expression of $\mathrm{P} 450$ aromatase and $17 \beta$-hydroxysteroid dehydrogenase type 1 at fetal-maternal interface during tubal pregnancy. J. Steroid Biochem. Mol. Biol. 87, 241-246. doi: 10.1016/j.jsbmb.2003.09.013

Lilla, C., Risch, A., Verla-Tebit, E., Hoffmeister, M., Brenner, H., and ChangClaude, J. (2007). SULT1A1 genotype and susceptibility to colorectal cancer. Int. J. Cancer 120, 201-206. doi: 10.1002/ijc.22156

Lim, Y. A., Grimm, A., Giese, M., Mensah-Nyagan, A. G., Villafranca, J. E., Ittner, L. M., et al. (2011). Inhibition of the mitochondrial enzyme ABAD restores the amyloid-beta-mediated deregulation of estradiol. PLoS ONE 6:e28887. doi: 10.1371/journal.pone.0028887

Lin, H. K., Jez, J. M., Schlegel, B. P., Peehl, D. M., Pachter, J. A., and Penning, T. M. (1997). Expression and characterization of recombinant type 2, $3 \alpha$ hydroxysteroid dehydrogenase (HSD) from human prostate: demonstration of bifunctional $3 \alpha / 17 \beta-H S D$ activity and cellular distribution. Mol. Endocrinol. 11, 1971-1984.

Lindemann, K., Malander, S., Christensen, R. D., Mirza, M. R., Kristensen, G. B., Aavall-Lundqvist, E., et al. (2014). Examestane in advanced or recurrent endometrial carcinoma: a prospective phase II study by the Nordic Society of Gynecologic Oncology (NSGO). BMC Cancer 14:68. doi: 10.1186/1471-2407-14-68

Liu, Y., Yao, Z. X., Bendavid, C., Borgmeyer, C., Han, Z., Cavalli, L. R., et al. (2005). Haploinsufficiency of cytochrome P450, 17ß-hydroxylase/17,20 lyase (CYP17) causes infertility in male mice. Mol. Endocrinol. 19, 2380-2389. doi: 10.1210/me.2004-0418

Lønning, P. E., and Eikesdal, H. P. (2013). Aromatase inhibition 2013: clinical state of the art and questions that remain to be solved. Endocr. Relat. Cancer 20, R183-R201. doi: 10.1530/ERC-13-0099

López, M., and Tena-Sempere, M. (2015). Estrogens and the control of energy homeostasis: a brain perspective. Trends Endocrinol. Metab. 26, 411-421. doi: $10.1016 /$ j.tem.2015.06.003

López, M., and Tena-Sempere, M. (2016). Estradiol and brown fat. Best Pract. Res. Clin. Endocrinol. Metab. 30, 527-536. doi: 10.1016/j.beem.2016.08.004

Loria, R. M., and Graf, M. R. (2012). 17 $\alpha$-androstenediol-mediated oncophagy of tumor cells by different mechanisms is determined by the target tumor. Ann. N. Y. Acad. Sci. 1262, 127-133. doi: 10.1111/j.1749-6632.2012.06602.x

Loriot, Y., Fizazi, K., Jones, R. J., Van den Brande, J., Molife, R. L., Omlin, A., et al. (2014). Safety, tolerability and anti-tumour activity of the androgen biosynthesis inhibitor ASP9521 in patients with metastatic castration-resistant prostate cancer: multi-centre phase I/II study. Invest. New Drugs 32, 995-1004. doi: 10.1007/s10637-014-0101-x

Loth, D. W., Soler Artigas, M., Gharib, S. A., Wain, L. V., Franceschini, N., Koch, B., et al. (2014). Genome-wide association analysis identifies six new loci associated with forced vital capacity. Nat. Genet. 46, 669-677. doi: 10.1038/ng.3011

Loza, M. C. (1995). Hydrolysis of estrone sulfate in uterine minces of the 6-days pregnant rat. J. Steroid Biochem. Mol. Biol. 52, 277-280. doi: 10.1016/0960-0760(94)00177-N
Lu, C., Lee, H. S., Pappas, G. P., Dilling, D. F., Burger, C. D., Shifren, A., et al. (2017). A Phase II Clinical Trial of an Aromatase Inhibitor for Postmenopausal Women with Lymphangioleiomyomatosis. Ann. Am. Thorac. Soc. 14, 919-928. doi: 10.1513/AnnalsATS.201610-824OC

Luchetti, S., Bossers, K., Van de Bilt, S., Agrapart, V., Morales, R. R., Frajese, G. V., et al. (2011). Neurosteroid biosynthetic pathways changes in prefrontal cortex in Alzheimer's disease. Neurobiol. Aging 32, 1964-1976. doi: 10.1016/j.neurobiolaging.2009.12.014

Lundin, E., Wirgin, I., Lukanova, A., Afanasyeva, Y., Krogh, V., Axelsson, T., et al. (2012). Selected polymorphisms in sex hormone-related genes, circulating sex hormones and risk of endometrial cancer. Cancer Epidemiol. 36, 445-452. doi: 10.1016/j.canep.2012.04.006

Luo, L., Deng, J., Wang, D. X., He, J., and Deng, W. (2015). Regulation of epithelial sodium channel expression by oestradiol and progestogen in alveolar epithelial cells. Respir. Physiol. Neurobiol. 216, 52-62. doi: 10.1016/j.resp.2015.06.001

Luu-The, V., Belanger, A., and Labrie, F. (2008). Androgen biosynthetic pathways in the human prostate. Best Pract. Res. Clin. Endocrinol. Metab. 22, 207-221. doi: 10.1016/j.beem.2008.01.008

Luu-The, V., and Labrie, F. (2010). The intracrine sex steroid biosynthesis pathways. Prog. Brain Res. 181, 177-192. doi: 10.1016/S0079-6123(08)81010-2

Ma, B. B., Oza, A., Eisenhauer, E., Stanimir, G., Carey, M., Chapman, W., et al. (2004). The activity of letrozole in patients with advanced or recurrent endometrial cancer and correlation with biological markers-a study of the National Cancer Institute of Canada Clinical Trials Group. Int. J. Gynecol. Cancer 14, 650-658. doi: 10.1111/j.1048-891X.2004.14419.x

Ma, W. G., Song, H., Das, S. K., Paria, B. C., and Dey, S. K. (2003). Estrogen is a critical determinant that specifies the duration of the window of uterine receptivity for implantation. Proc. Natl. Acad. Sci. U.S.A. 100, 2963-2968. doi: 10.1073/pnas.0530162100

MacKenzie, S. M., Dewar, D., Stewart, W., Fraser, R., Connell, J. M., and Davies, E. (2008). The transcription of steroidogenic genes in the human cerebellum and hippocampus: a comparative survey of normal and Alzheimer's tissue. J. Endocrinol. 196, 123-130. doi: 10.1677/JOE-07-0427

Maentausta, O., Peltoketo, H., Isomaa, V., Jouppila, P., and Vihko, R. (1990). Immunological measurement of human $17 \beta$-hydroxysteroid dehydrogenase. J. Steroid. Biochem. 36, 673-680.

Mäentausta, O., Sormunen, R., Isomaa, V., Lehto, V. P., Jouppila, P., and Vihko, R. (1991). Immunohistochemical localization of 17 beta-hydroxysteroid dehydrogenase in the human endometrium during the menstrual cycle. Lab. Invest. 65, 582-587.

Mahendroo, M. S., Cala, K. M., Hess, D. L., and Russell, D. W. (2001). Unexpected virilization in male mice lacking steroid 5 alpha-reductase enzymes. Endocrinology 142, 4652-4662. doi: 10.1210/endo.142.11.8510

Mahendroo, M. S., Cala, K. M., and Russell, D. W. (1996). 5 alpha-reduced androgens play a key role in murine parturition. Mol. Endocrinol. 10, 380-392.

Maia, H. Jr., Pimentel, K., Casoy, J., Correia, T., Freitas, L. A., Zausner, B., et al. (2007). Aromatase expression in the eutopic endometrium of myomatous uteri: the influence of the menstrual cycle and oral contraceptive use. Gynecol. Endocrinol. 23, 320-324. doi: 10.1080/09513590701321565

Maia, H. Jr., Pimentel, K., Silva, T. M., Freitas, L. A., Zausner, B., Athayde, C., et al. (2006). Aromatase and cyclooxygenase-2 expression in endometrial polyps during the menstrual cycle. Gynecol. Endocrinol. 22, 219-224. doi: 10.1080/09513590600585955

Maltais, R., and Poirier, D. (2011). Steroid sulfatase inhibitors: a review covering the promising 2000-2010 decade. Steroids 76, 929-948. doi: 10.1016/j.steroids.2011.03.010

Manenda, M. S., Hamel, C. J., Masselot-Joubert, L., Picard, M. E., and Shi, R. (2016). Androgen-metabolizing enzymes: A structural perspective. J. Steroid Biochem. Mol. Biol. 161, 54-72. doi: 10.1016/j.jsbmb.2016 02.021

Marchais-Oberwinkler, S., Wetzel, M., Ziegler, E., Kruchten, P., Werth, R., Henn, C., et al. (2011). New drug-like hydroxyphenylnaphthol steroidomimetics as potent and selective $17 \beta$-hydroxysteroid dehydrogenase type 1 inhibitors for the treatment of estrogen-dependent diseases. J. Med. Chem. 54, 534-547. doi: $10.1021 /$ jm 1009082

Masi, L., Becherini, L., Gennari, L., Amedei, A., Colli, E., Falchetti, A., et al. (2001). Polymorphism of the aromatase gene in postmenopausal Italian 
women: distribution and correlation with bone mass and fracture risk. J. Clin. Endocrinol. Metab. 86, 2263-2269. doi: 10.1210/jc.86.5.2263

Matsunaga, T., Hojo, A., Yamane, Y., Endo, S., El-Kabbani, O., and Hara, A. (2013). Pathophysiological roles of aldo-keto reductases (AKR1C1 and AKR1C3) in development of cisplatin resistance in human colon cancers. Chem. Biol. Interact. 202, 234-242. doi: 10.1016/j.cbi.2012.09.024

Matsuzaki, S., Canis, M., Pouly, J. L., Dechelotte, P. J., and Mage, G. (2006). Analysis of aromatase and $17 \beta$-hydroxysteroid dehydrogenase type 2 messenger ribonucleic acid expression in deep endometriosis and eutopic endometrium using laser capture microdissection. Fertil. Steril. 85, 308-313. doi: $10.1016 /$ j.fertnstert.2005.08.017

Mauvais-Jarvis, F., Clegg, D. J., and Hevener, A. L. (2013). The role of estrogens in control of energy balance and glucose homeostasis. Endocr. Rev. 34, 309-338. doi: 10.1210/er.2012-1055

McCarthy, M. (2000). The "gender gap" in autoimmune disease. Lancet 356, 1088. doi: 10.1016/S0140-6736(05)74535-9

McEwen, B. S., and Milner, T. A. (2017). Understanding the broad influence of sex hormones and sex differences in the brain. J. Neurosci. Res. 95, 24-39. doi: 10.1002/jnr.23809

Meijer, D., Gelderblom, H., Karperien, M., Cleton-Jansen, A. M., Hogendoorn, P. C., and Bovee, J. V. (2011). Expression of aromatase and estrogen receptor alpha in chondrosarcoma, but no beneficial effect of inhibiting estrogen signaling both in vitro and in vivo. Clin. Sarcoma Res. 1:5. doi: 10.1186/2045-3329-1-5

Meloche, C. A., and Falany, C. N. (2001). Expression and characterization of the human 3 beta-hydroxysteroid sulfotransferases (SULT2B1a and SULT2B1b). J. Steroid Biochem. Mol. Biol. 77, 261-269. doi: 10.1016/S0960-0760(01)00064-4

Miki, Y., Nakata, T., Suzuki, T., Darnel, A. D., Moriya, T., Kaneko, C., et al. (2002). Systemic distribution of steroid sulfatase and estrogen sulfotransferase in human adult and fetal tissues. J. Clin. Endocrinol. Metab. 87, 5760-5768. doi: $10.1210 /$ jc.2002-020670

Milewich, L., Garcia, R. L., and Johnson, A. R. (1983). Steroid sulfatase activity in human lung tissue and in endothelial pulmonary cells in culture. J. Clin. Endocrinol. Metab. 57, 8-14. doi: 10.1210/jcem-57-1-8

Miller, P. B., Parnell, B. A., Bushnell, G., Tallman, N., Forstein, D. A., Higdon, H. L III., et al. (2012a). Endometrial receptivity defects during IVF cycles with and without letrozole. Hum. Reprod. 27, 881-888. doi: 10.1093/humrep/der452

Miller, V. L., Lin, H. K., Murugan, P., Fan, M., Penning, T. M., Brame, L. S., et al. (2012b). Aldo-keto reductase family 1 member C3 (AKR1C3) is expressed in adenocarcinoma and squamous cell carcinoma but not small cell carcinoma. Int. J. Clin. Exp. Pathol. 5, 278-289.

Miller, W. L., and Auchus, R. J. (2011). The molecular biology, biochemistry, and physiology of human steroidogenesis and its disorders. Endocr. Rev. 32, 81-151. doi: 10.1210/er.2010-0013

Mindnich, R., Moller, G., and Adamski, J. (2004). The role of 17 betahydroxysteroid dehydrogenases. Mol. Cell. Endocrinol. 218, 7-20. doi: 10.1016/j.mce.2003.12.006

Miyaura, C., Toda, K., Inada, M., Ohshiba, T., Matsumoto, C., Okada, T., et al. (2001). Sex- and age-related response to aromatase deficiency in bone. Biochem. Biophys. Res. Commun. 280, 1062-1068. doi: 10.1006/bbrc.2001.4246

Moeller, G., and Adamski, J. (2006). Multifunctionality of human 17ßhydroxysteroid dehydrogenases. Mol. Cell. Endocrinol. 248, 47-55. doi: $10.1016 /$ j.mce.2005.11.031

Moeller, G., and Adamski, J. (2009). Integrated view on 17 $\beta$ hydroxysteroid dehydrogenases. Mol. Cell. Endocrinol. 301, 7-19. doi: 10.1016/j.mce.2008.10.040

Möller, G., Leenders, F., van Grunsven, E. G., Dolez, V., Qualmann, B., Kessels, M. M., et al. (1999). Characterization of the HSD17B4 gene: D-specific multifunctional protein $2 / 17 \beta$-hydroxysteroid dehydrogenase IV. J. Steroid Biochem. Mol. Biol. 69, 441-446. doi: 10.1016/S0960-0760(99)00066-7

Morgat, A., Axelsen, K. B., Lombardot, T., Alcantara, R., Aimo, L., Zerara, M., et al. (2015). Updates in Rhea-a manually curated resource of biochemical reactions. Nucleic Acids Res. 43, D459-D464. doi: 10.1093/nar/gku961

Mori, T., Ito, F., Matsushima, H., Takaoka, O., Koshiba, A., Tanaka, Y., et al. (2015). Dienogest reduces HSD17 $\beta 1$ expression and activity in endometriosis. J. Endocrinol. 225, 69-76. doi: 10.1530/JOE-15-0052

Morice, P., Leary, A., Creutzberg, C., Abu-Rustum, N., and Darai, E. (2015). Endometrial cancer. Lancet 387, 1094-1108. doi: 10.1016/S0140-6736(15)00130-0
Morishima, A., Grumbach, M. M., Simpson, E. R., Fisher, C., and Qin, K. (1995). Aromatase deficiency in male and female siblings caused by a novel mutation and the physiological role of estrogens. J. Clin. Endocrinol. Metab. 80, 3689-3698.

Mueller, J. W., Gilligan, L. C., Idkowiak, J., Arlt, W., and Foster, P. A. (2015). The regulation of steroid action by sulfation and desulfation. Endocr. Rev. 36, 526-563. doi: 10.1210/er.2015-1036

Muir, M., Romalo, G., Wolf, L., Elger, W., and Schweikert, H. U. (2004). Estrone sulfate is a major source of local estrogen formation in human bone. J. Clin. Endocrinol. Metab. 89, 4685-4692. doi: 10.1210/jc.2004-0049

Mukai, H., Tsurugizawa, T., Ogiue-Ikeda, M., Murakami, G., Hojo, Y., Ishii, $\mathrm{H}$, et al. (2006). Local neurosteroid production in the hippocampus: influence on synaptic plasticity of memory. Neuroendocrinology 84, 255-263. doi: 10.1159/000097747

Mullis, P. E., Yoshimura, N., Kuhlmann, B., Lippuner, K., Jaeger, P., and Harada, H. (1997). Aromatase deficiency in a female who is compound heterozygote for two new point mutations in the P450arom gene: impact of estrogens on hypergonadotropic hypogonadism, multicystic ovaries, and bone densitometry in childhood. J. Clin. Endocrinol. Metab. 82, 1739-1745. doi: $10.1210 /$ jc.82.6.1739

Munroe, D. G., and Chang, P. L. (1987). Tissue-specific expression of human arylsulfatase-C isozymes and steroid sulfatase. Am. J. Hum. Genet. 40, 102-114.

Murakami, G., Tanabe, N., Ishii, H. T., Ogiue-Ikeda, M., Tsurugizawa, T., Mukai, H., et al. (2006). Role of cytochrome p450 in synaptocrinology: endogenous estrogen synthesis in the brain hippocampus. Drug Metab. Rev. 38, 353-369. doi: 10.1080/03602530600724068

Musatov, S., Chen, W., Pfaff, D. W., Mobbs, C. V., Yang, X. J., Clegg, D. J., et al. (2007). Silencing of estrogen receptor alpha in the ventromedial nucleus of hypothalamus leads to metabolic syndrome. Proc. Natl. Acad. Sci. U.S.A. 104, 2501-2506. doi: 10.1073/pnas.0610787104

Nadal, A., Alonso-Magdalena, P., Soriano, S., Ripoll, C., Fuentes, E., Quesada, I., et al. (2011). Role of estrogen receptors alpha, beta and GPER1/GPR30 in pancreatic beta-cells. Front Biosci (Landmark Ed) 16, 251-260. doi: $10.2741 / 3686$

Naftolin, F., Horvath, T. L., Jakab, R. L., Leranth, C., Harada, N., and Balthazart, J. (1996). Aromatase immunoreactivity in axon terminals of the vertebrate brain. An immunocytochemical study on quail, rat, monkey and human tissues. Neuroendocrinology 63, 149-155. doi: 10.1159/000126951

Naftolin, F., and MacLusky, N. J. (1982). Aromatase in the central nervous system. Cancer Res 42, 3274s-3276s.

Nakarai, C., Osawa, K., Akiyama, M., Matsubara, N., Ikeuchi, H., Yamano, T., et al. (2015). Expression of AKR1C3 and CNN3 as markers for detection of lymph node metastases in colorectal cancer. Clin. Exp. Med. 15, 333-341. doi: 10.1007/s10238-014-0298-1

Nayeb-Hashemi, H., and Kaunitz, J. D. (2009). Gastroduodenal mucosal defense. Curr. Opin. Gastroenterol. 25, 537-543. doi: 10.1097/MOG.0b013e328330da7b

Nemoto, Y., Toda, K., Ono, M., Fujikawa-Adachi, K., Saibara, T., Onishi, S., et al. (2000). Altered expression of fatty acid-metabolizing enzymes in aromatasedeficient mice. J. Clin. Invest. 105, 1819-1825. doi: 10.1172/JCI9575

Niikawa, H., Suzuki, T., Miki, Y., Suzuki, S., Nagasaki, S., Akahira, J., et al. (2008). Intratumoral estrogens and estrogen receptors in human non-small cell lung carcinoma. Clin. Cancer Res. 14, 4417-4426. doi: 10.1158/1078-0432.CCR-07-1950

Nishimura, M., and Naito, S. (2006). Tissue-specific mRNA expression profiles of human phase I metabolizing enzymes except for cytochrome P450 and phase II metabolizing enzymes. Drug Metab. Pharmacokinet. 21, 357-374. doi: $10.2133 / \mathrm{dmpk} .21 .357$

Noble, L. S., Simpson, E. R., Johns, A., and Bulun, S. E. (1996). Aromatase expression in endometriosis. J. Clin. Endocrinol. Metab. 81, 174-179.

Noble, L. S., Takayama, K., Zeitoun, K. M., Putman, J. M., Johns, D. A., Hinshelwood, M. M., et al. (1997). Prostaglandin E2 stimulates aromatase expression in endometriosis-derived stromal cells. J. Clin. Endocrinol. Metab. 82, 600-606. doi: 10.1210/jc.82.2.600

Noël, J. C., Anaf, V., Borghese, B., Vaiman, D., Fayt, I., and Chapron, C. (2011). The steroidogenic factor-1 protein is not expressed in various forms of endometriosis but is strongly present in ovarian cortical or medullary mesenchymatous cells adjacent to endometriotic foci. Fertil. Steril. 95, 2655-2657. doi: 10.1016/j.fertnstert.2011.01.131 
Nokelainen, P., Peltoketo, H., Mustonen, M., and Vihko, P. (2000). Expression of mouse $17 \beta$-hydroxysteroid dehydrogenase/17-ketosteroid reductase type 7 in the ovary, uterus, and placenta: localization from implantation to late pregnancy. Endocrinology 141, 772-778. doi: 10.1210/endo.141.2.7309

Oduwole, O. O., Isomaa, V. V., Nokelainen, P. A., Stenback, F., and Vihko, P. T. (2002). Downregulation of estrogen-metabolizing 17 betahydroxysteroid dehydrogenase type 2 expression correlates inversely with Ki67 proliferation marker in colon-cancer development. Int. J. Cancer 97, 1-6. doi: $10.1002 /$ ijc. 1567

Oduwole, O. O., Makinen, J. M., Isomaa, V. V., Karttunen, T. J., and Vihko, P. T. (2003a). Sex steroid metabolism in human gastric mucosa: 17 betahydroxysteroid dehydrogenase type 2 in normal, inflamed and neoplastic gastric tissues. Anticancer Res. 23, 3889-3897.

Oduwole, O. O., Makinen, M. J., Isomaa, V. V., Pulkka, A., Jernvall, P., Karttunen, T. J., et al. (2003b). 17 $\beta$-hydroxysteroid dehydrogenase type 2: independent prognostic significance and evidence of estrogen protection in female patients with colon cancer. J. Steroid Biochem. Mol. Biol. 87, 133-140. doi: 10.1016/j.jsbmb.2003.08.008

Ohno, S., Nishikawa, K., Honda, Y., and Nakajin, S. (2008). Expression in E. coli and tissue distribution of the human homologue of the mouse Ke 6 gene, $17 \beta$-hydroxysteroid dehydrogenase type 8. Mol. Cell. Biochem. 309, 209-215. doi: 10.1007/s11010-007-9637-9

Olson, S. H., Bandera, E. V., and Orlow, I. (2007). Variants in estrogen biosynthesis genes, sex steroid hormone levels, and endometrial cancer: a HuGE review. Am. J. Epidemiol. 165, 235-245. doi: 10.1093/aje/kwk015

Osinski, M., Wirstlein, P., Wender-Ozegowska, E., Mikolajczyk, M., Jagodzinski, P. P., and Szczepanska, M. (2018). HSD3B2, HSD17B1, HSD17B2, ESR1, ESR2 and AR expression in infertile women with endometriosis. Ginekol. Pol. 89, 125-134. doi: 10.5603/GP.a2018.0022

Oz, O. K., Millsaps, R., Welch, R., Birch, J., and Zerwekh, J. E. (2001). Expression of aromatase in the human growth plate. J. Mol. Endocrinol. 27, 249-253. doi: $10.1677 /$ jme.0.0270249

Oz, O. K., Zerwekh, J. E., Fisher, C., Graves, K., Nanu, L., Millsaps, R., et al. (2000). Bone has a sexually dimorphic response to aromatase deficiency. J. Bone Miner. Res. 15, 507-514. doi: 10.1359/jbmr.2000.15.3.507

Palmer, B. F., and Clegg, D. J. (2015). The sexual dimorphism of obesity. Mol. Cell. Endocrinol. 402, 113-119. doi: 10.1016/j.mce.2014.11.029

Palmieri, C., Stein, R. C., Liu, X., Hudson, E., Nicholas, H., Sasano, H., et al. (2017a). IRIS study: a phase II study of the steroid sulfatase inhibitor Irosustat when added to an aromatase inhibitor in ER-positive breast cancer patients. Breast Cancer Res. Treat. 165, 343-353. doi: 10.1007/s10549-0174328-z

Palmieri, C., Szydlo, R., Miller, M., Barker, L., Patel, N. H., Sasano, H., et al. (2017b). IPET study: an FLT-PET window study to assess the activity of the steroid sulfatase inhibitor irosustat in early breast cancer. Breast Cancer Res. Treat. 166, 527-539. doi: 10.1007/s10549-017-4427-x

Park, A. L., Lin, H. K., Yang, Q., Sing, C. W., Fan, M., Mapstone, T. B., et al. (2010).

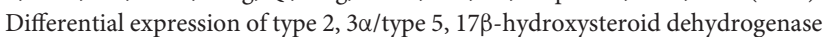
(AKR1C3) in tumors of the central nervous system. Int. J. Clin. Exp. Pathol. 3, 743-754.

Park, Y. S., Gauna, A. E., and Cha, S. (2015). Mouse models of primary Sjogren's syndrome. Curr. Pharm. Des. 21, 2350-2364. doi: 10.2174/1381612821666150316120024

Pathirage, N., Di Nezza, L. A., Salmonsen, L. A., Jobling, T., Simpson, E. R., and Clyne, C. D. (2006). Expression of aromatase, estrogen receptors, and their coactivators in patients with endometrial cancer. Fertil. Steril. 86, 469-472. doi: 10.1016/j.fertnstert.2005.12.057

Pautier, P., Vergote, I., Joly, F., Melichar, B., Kutarska, E., Hall, G., et al. (2017). A Phase 2, randomized, open-label study of irosustat versus megestrol acetate in advanced endometrial cancer. Int. J. Gynecol. Cancer 27, 258-266. doi: 10.1097/IGC.0000000000000862

Pearson Murphy, B. E., Steinberg, S. I., Hu, F. Y., and Allison, C. M. (2001). Neuroactive ring A-reduced metabolites of progesterone in human plasma during pregnancy: elevated levels of 5 alpha-dihydroprogesterone in depressed patients during the latter half of pregnancy. J. Clin. Endocrinol. Metab. 86, 5981-5987. doi: 10.1210/jcem.86.12.8122

Pelletier, G., Luu-The, V., Tetu, B., and Labrie, F. (1999). Immunocytochemical localization of type 5, 17ß-hydroxysteroid dehydrogenase in human reproductive tissues. J. Histochem. Cytochem. 47, 731-738. doi: 10.1177/002215549904700602

Penning, T. M. (2017). Aldo-Keto Reductase (AKR) 1C3 inhibitors: a patent review. Expert Opin. Ther. Pat. 27, 1329-1340. doi: 10.1080/13543776.2017.1379503

Penning, T. M., Burczynski, M. E., Jez, J. M., Hung, C. F., Lin, H. K., Ma, H., et al. (2000). Human $3 \alpha$-hydroxysteroid dehydrogenase isoforms (AKR1C1AKR1C4) of the aldo-keto reductase superfamily: functional plasticity and tissue distribution reveals roles in the inactivation and formation of male and female sex hormones. Biochem. J. 351, 67-77. doi: 10.1042/bj3510067

Penning, T. M., Jin, Y., Steckelbroeck, S., Lanisnik Rizner, T., and Lewis, M. (2004). Structure-function of human 3 alpha-hydroxysteroid dehydrogenases: genes and proteins. Mol. Cell. Endocrinol. 215, 63-72. doi: 10.1016/j.mce.2003. 11.006

Perez Carrion, R., Alberola Candel, V., Calabresi, F., Michel, R. T., Santos, R., Delozier, T., et al. (1994). Comparison of the selective aromatase inhibitor formestane with tamoxifen as first-line hormonal therapy in postmenopausal women with advanced breast cancer. Ann. Oncol. 5(Suppl. 7), S19-S24.

Pezzi, V., Mathis, J. M., Rainey, W. E., and Carr, B. R. (2003). Profiling transcript levels for steroidogenic enzymes in fetal tissues. J. Steroid Biochem. Mol. Biol. 87, 181-189. doi: 10.1016/j.jsbmb.2003.07.006

Piccinato, C. A., Neme, R. M., Torres, N., Sanches, L. R., Cruz Derogis, P. B., Brudniewski, H. F., et al. (2016a). Increased expression of CYP1A1 and CYP1B1 in ovarian/peritoneal endometriotic lesions. Reproduction 151, 683-692. doi: 10.1530/REP-15-0581

Piccinato, C. A., Neme, R. M., Torres, N., Sanches, L. R., Derogis, P., Brudniewski, H. F., et al. (2016b). Effects of steroid hormone on estrogen sulfotransferase and on steroid sulfatase expression in endometriosis tissue and stromal cells. J. Steroid Biochem. Mol. Biol. 158, 117-126. doi: 10.1016/j.jsbmb.2015.12.025

Piekorz, R. P., Gingras, S., Hoffmeyer, A., Ihle, J. N., and Weinstein, Y. (2005). Regulation of progesterone levels during pregnancy and parturition by signal transducer and activator of transcription 5 and $20 \alpha$-hydroxysteroid dehydrogenase. Mol. Endocrinol. 19, 431-440. doi: 10.1210/me.2004-0302

Platia, M. P., Fencl, M. D., Elkind-Hirsch, K. E., Canick, J. A., and Tulchinsky, D. (1984). Estrone sulfatase activity in the human brain and estrone sulfate levels in the normal menstrual cycle. J. Steroid Biochem. 21, 237-241. doi: 10.1016/0022-4731(84)90275-9

Pohl, O., Bestel, E., and Gotteland, J. P. (2014). Synergistic effects of E2MATE and norethindrone acetate on steroid sulfatase inhibition: a randomized phase I proof-of-principle clinical study in women of reproductive age. Reprod. Sci. 21, 1256-1265. doi: 10.1177/1933719114522526

Pollow, K., Lubbert, H., Jeske, R., and Pollow, B. (1975a). Studies on 17 $\beta$ hydroxysteroid dehydrogenase in human endometrium and endometrial carcinoma. Acta Endocrinol. 79, 146-156.

Pollow, K., Lubbert, H., and Pollow, B. (1975b). Studies on 17 beta-hydroxysteroid dehydrogenase in human endometrium and endometrial carcinoma. III. Partial purification and characterization of the microsomal enzyme. Acta Endocrinol (Copenh) 80, 355-364.

Pollow, K., Lubbert, H., and Pollow, B. (1976). On the mitochondrial 17 $\beta$ hydroxysteroid dehydrogenase from human endometrium and endometrial carcinoma: characterization and intramitochondrial distribution. J. Steroid Biochem. 7, 45-50. doi: 10.1016/0022-4731(76)90163-1

Polow, K., Lubbert, H., Boquoi, E., Kreutzer, G., Jeske, R., and Pollow, B. (1975). Studies on $17 \beta$-hydroxysteroid dehydrogenase in human endometrium and endometrial carcinoma I. Subcellular distribution and variations of specific enzyme activity. Acta Endocrinol (Copenh) 79, 134-145.

Prehn, C., Moller, G., and Adamski, J. (2009). Recent advances in $17 \beta$ hydroxysteroid dehydrogenases. J. Steroid Biochem. Mol. Biol. 114, 72-77. doi: 10.1016/j.jsbmb.2008.12.024

Prizant, H., and Hammes, S. R. (2016). Minireview: lymphangioleiomyomatosis (LAM): the "Other" steroid-sensitive cancer. Endocrinology 157, 3374-3383. doi: 10.1210/en.2016-1395

Prossnitz, E. R., and Barton, M. (2011). The G-protein-coupled estrogen receptor GPER in health and disease. Nat. Rev. Endocrinol. 7, 715-726. doi: $10.1038 /$ nrendo.2011.122

Prost, O., and Adessi, G. L. (1983). Estrone and dehydroepiandrosterone sulfatase activities in normal and pathological human endometrium biopsies. J. Clin. Endocrinol. Metab. 56, 653-661. doi: 10.1210/jcem-56-4-653 
Purohit, A., Flanagan, A. M., and Reed, M. J. (1992). Estrogen synthesis by osteoblast cell lines. Endocrinology 131, 2027-2029. doi: 10.1210/endo.131.4.1396346

Purohit, A., and Foster, P. A. (2012). Steroid sulfatase inhibitors for estrogen- and androgen-dependent cancers. J. Endocrinol. 212, 99-110. doi: 10.1530/JOE-11-0266

Purohit, A., Fusi, L., Brosens, J., Woo, L. W., Potter, B. V., and Reed, M. J. (2008). Inhibition of steroid sulphatase activity in endometriotic implants by 667 COUMATE: a potential new therapy. Hum. Reprod. 23, 290-297. doi: 10.1093/humrep/dem308

Qaiser, M. Z., Dolman, D. E. M., Begley, D. J., Abbott, N. J., Cazacu-Davidescu, M., Corol, D. I., et al. (2017). Uptake and metabolism of sulphated steroids by the blood-brain barrier in the adult male rat. J. Neurochem. 142, 672-685. doi: 10.1111/jnc.14117

Qian, Y. M., Sun, X. J., Tong, M. H., Li, X. P., Richa, J., and Song, W. C. (2001). Targeted disruption of the mouse estrogen sulfotransferase gene reveals a role of estrogen metabolism in intracrine and paracrine estrogen regulation. Endocrinology 142, 5342-5350. doi: 10.1210/endo.142. 12.8540

Qin, K., Ehrmann, D. A., Cox, N., Refetoff, S., and Rosenfield, R. L. (2006). Identification of a functional polymorphism of the human type $5,17 \beta$ hydroxysteroid dehydrogenase gene associated with polycystic ovary syndrome. J. Clin. Endocrinol. Metab. 91, 270-276. doi: 10.1210/jc. 2005-2012

Quinkler, M., Sinha, B., Tomlinson, J. W., Bujalska, I. J., Stewart, P. M., and Arlt, W. (2004). Androgen generation in adipose tissue in women with simple obesity-a site-specific role for $17 \beta$-hydroxysteroid dehydrogenase type 5. J. Endocrinol. 183, 331-342. doi: 10.1677/joe.1.05762

Rajkowski, K. M., Robel, P., and Baulieu, E. E. (1997). Hydroxysteroid sulfotransferase activity in the rat brain and liver as a function of age and sex. Steroids 62, 427-436. doi: 10.1016/S0039-128X(97)00013-5

Rantakari, P., Lagerbohm, H., Kaimainen, M., Suomela, J. P., Strauss, L., Sainio, K., et al. (2010). Hydroxysteroid (17\{beta\}) dehydrogenase 12 is essential for mouse organogenesis and embryonic survival. Endocrinology 151, 1893-1901. doi: 10.1210/en.2009-0929

Rantakari, P., Strauss, L., Kiviranta, R., Lagerbohm, H., Paviala, J., Holopainen, I., et al. (2008). Placenta defects and embryonic lethality resulting from disruption of mouse hydroxysteroid (17-beta) dehydrogenase 2 gene. Mol. Endocrinol. 22, 665-675. doi: 10.1210/me.2007-0257

Rauschenberger, K., Scholer, K., Sass, J. O., Sauer, S., Djuric, Z., Rumig, C., et al. (2010). A non-enzymatic function of $17 \beta$-hydroxysteroid dehydrogenase type 10 is required for mitochondrial integrity and cell survival. EMBO Mol. Med. 2, 51-62. doi: 10.1002/emmm.200900055

Rawłuszko, A. A., Horbacka, K., Krokowicz, P., and Jagodzinski, P. P. (2011). Decreased expression of $17 \beta$-hydroxysteroid dehydrogenase type 1 is associated with DNA hypermethylation in colorectal cancer located in the proximal colon. $B M C$ Cancer 11:522. doi: 10.1186/1471-2407-11-522

Reddy, D. S. (2010). Neurosteroids: endogenous role in the human brain and therapeutic potentials. Prog. Brain Res. 186, 113-137. doi: 10.1016/B978-0-444-53630-3.00008-7

Reed, M. J., Purohit, A., Woo, L. W., Newman, S. P., and Potter, B. V. (2005). Steroid sulfatase: molecular biology, regulation, and inhibition. Endocr. Rev. 26, 171-202. doi: 10.1210/er.2004-0003

Rege, J., Karashima, S., Lerario, A. M., Smith, J. M., Auchus, R. J., Kasa-Vubu, J. Z., et al. (2016). Age-dependent increases in adrenal cytochrome b5 and serum 5-androstenediol-3-sulfate. J. Clin. Endocrinol. Metab. 101, 4585-4593. doi: 10.1210/jc.2016-2864

Rennert, G., Rennert, H. S., Pinchev, M., Lavie, O., and Gruber, S. B. (2009). Use of hormone replacement therapy and the risk of colorectal cancer. J. Clin. Oncol. 27, 4542-4547. doi: 10.1200/JCO.2009.22.0764

Rhee, H. S., Oh, S. H., Ko, B. J., Han, D. M., Jeon, B. H., Park, H., et al. (2003). Expression of $3 \beta$-hydroxysteroid dehydrogenase and P450 side chain cleavage enzyme in the human uterine endometrium. Exp. Mol. Med. 35, 160-166. doi: 10.1038/emm.2003.22

Riches, Z., Stanley, E. L., Bloomer, J. C., and Coughtrie, M. W. (2009). Quantitative evaluation of the expression and activity of five major sulfotransferases (SULTs) in human tissues: the SULT "pie". Drug Metab. Dispos. 37, 2255-2261. doi: $10.1124 / \mathrm{dmd} .109 .028399$
Riggs, B. L., Khosla, S., and Melton, L. J. III. (2002). Sex steroids and the construction and conservation of the adult skeleton. Endocr. Rev. 23, 279-302. doi: 10.1210/edrv.23.3.0465

Rižner, T. L. (2013). Estrogen biosynthesis, phase I and phase II metabolism, and action in endometrial cancer. Mol. Cell. Endocrinol. 381, 124-139. doi: 10.1016/j.mce.2013.07.026

Rižner, T. L. (2016). The important roles of steroid sulfatase and sulfotransferases in gynecological diseases. Front. Pharmacol. 7:30. doi: 10.3389/fphar.2016.00030

Rižner, T. L., and Penning, T. M. (2014). Role of aldo-keto reductase family 1 (AKR1) enzymes in human steroid metabolism. Steroids 79, 49-63. doi: 10.1016/j.steroids.2013.10.012

Rizner, T. L., Smuc, T., Rupreht, R., Sinkovec, J., and Penning, T. M. (2006). AKR1C1 and AKR1C3 may determine progesterone and estrogen ratios in endometrial cancer. Mol. Cell. Endocrinol. 248, 126-135. doi: $10.1016 /$ j.mce.2005.10.009

Rižner, T. L., Thalhammer, T., and Ozvegy-Laczka, C. (2017). The importance of steroid uptake and intracrine action in endometrial and ovarian cancers. Front. Pharmacol. 8:346. doi: 10.3389/fphar.2017.00346

Rodríguez-Sanz, M., Garcia-Giralt, N., Prieto-Alhambra, D., Servitja, S., Balcells, S., Pecorelli, R., et al. (2015). CYP11A1 expression in bone is associated with aromatase inhibitor-related bone loss. J. Mol. Endocrinol. 55, 69-79. doi: 10.1530/JME-15-0079

Rose, P. G., Brunetto, V. L., VanLe, L., Bell, J., Walker, J. L., and Lee, R. B. (2000). A phase II trial of anastrozole in advanced recurrent or persistent endometrial carcinoma: a Gynecologic Oncology Group study. Gynecol. Oncol. 78, 212-216. doi: 10.1006/gyno.2000.5865

Roshan, M. H., Tambo, A., and Pace, N. P. (2016). The role of testosterone in colorectal carcinoma: pathomechanisms and open questions. EPMA J. 7, 22. doi: 10.1186/s13167-016-0071-5

Rosner, W., Hankinson, S. E., Sluss, P. M., Vesper, H. W., and Wierman, M. E. (2013). Challenges to the measurement of estradiol: an endocrine society position statement. J. Clin. Endocrinol. Metab. 98, 1376-1387. doi: 10.1210/jc.2012-3780

Rubin, G. L., Harrold, A. J., Mills, J. A., Falany, C. N., and Coughtrie, M. W. (1999). Regulation of sulphotransferase expression in the endometrium during the menstrual cycle, by oral contraceptives and during early pregnancy. Mol. Hum. Reprod. 5, 995-1002. doi: 10.1093/molehr/5.11.995

Sahu, B., Sun, W., Perusek, L., Parmar, V., Le, Y. Z., Griswold, M. D., et al. (2015). Conditional ablation of retinol dehydrogenase 10 in the retinal pigmented epithelium causes delayed dark adaption in mice. J. Biol. Chem. 290, 27239-27247. doi: 10.1074/jbc.M115.682096

Saito, H., and Yanaihara, T. (1998). Steroid formation in osteoblast-like cells. J. Int. Med. Res. 26, 1-12. doi: 10.1177/030006059802600101

Saitoh, Y., Sasano, H., Naganuma, H., Ohtani, H., Sasano, N., Ohuchi, A., et al. (1992). De novo expression of aromatase in gastric carcinoma. Light and electron microscopic immunohistochemical and immunoblot study. Pathol. Res. Pract. 188, 53-60. doi: 10.1016/S0344-0338(11)81156-8

Sakiani, S., Olsen, N. J., and Kovacs, W. J. (2013). Gonadal steroids and humoral immunity. Nat. Rev. Endocrinol. 9, 56-62. doi: 10.1038/nrendo.2012.206

Sakurai, N., Miki, Y., Suzuki, T., Watanabe, K., Narita, T., Ando, K., et al. (2006). Systemic distribution and tissue localizations of human $17 \beta$ hydroxysteroid dehydrogenase type 12. J. Steroid Biochem. Mol. Biol. 99, 174-181. doi: 10.1016/j.jsbmb.2006.01.010

Salas, S., Jezequel, P., Campion, L., Deville, J. L., Chibon, F., Bartoli, C., et al. (2009). Molecular characterization of the response to chemotherapy in conventional osteosarcomas: predictive value of HSD17B10 and IFITM2. Int. J. Cancer 125, 851-860. doi: 10.1002/ijc.24457

Salman, E. D., Faye-Petersen, O., and Falany, C. N. (2011). Hydroxysteroid sulfotransferase $2 \mathrm{~B} 1 \mathrm{~b}$ expression and localization in normal human brain. Horm. Mol. Biol. Clin. Investig. 8, 445-454. doi: 10.1515/HMBCI.2011.117

Salman, E. D., Kadlubar, S. A., and Falany, C. N. (2009). Expression and localization of cytosolic sulfotransferase (SULT) $1 \mathrm{~A} 1$ and SULT1A3 in normal human brain. Drug Metab. Dispos. 37, 706-709. doi: 10.1124/dmd.108.025767

Saloniemi, T., Jarvensivu, P., Koskimies, P., Jokela, H., Lamminen, T., GhaemMaghami, S., et al. (2010). Novel hydroxysteroid (17ß) dehydrogenase 1 inhibitors reverse estrogen-induced endometrial hyperplasia in transgenic mice. Am. J. Pathol. 176, 1443-1451. doi: 10.2353/ajpath.2010.090325 
Sánchez-Guijo, A., Neunzig, J., Gerber, A., Oji, V., Hartmann, M. F., Schuppe, H. C., et al. (2016). Role of steroid sulfatase in steroid homeostasis and characterization of the sulfated steroid pathway: Evidence from steroid sulfatase deficiency. Mol. Cell. Endocrinol. 437, 142-153. doi: 10.1016/j.mce.2016. 08.019

Sasano, H., Takashashi, K., Satoh, F., Nagura, H., and Harada, N. (1998). Aromatase in the human central nervous system. Clin. Endocrinol. (Oxf). 48, 325-329. doi: 10.1046/j.1365-2265.1998.00390.x

Sasano, H., Uzuki, M., Sawai, T., Nagura, H., Matsunaga, G., Kashimoto, O., et al. (1997). Aromatase in human bone tissue. J. Bone Miner. Res. 12, 1416-1423. doi: 10.1359/jbmr.1997.12.9.1416

Sathish, V., Martin, Y. N., and Prakash, Y. S. (2015). Sex steroid signaling: implications for lung diseases. Pharmacol. Ther. 150, 94-108. doi: 10.1016/j.pharmthera.2015.01.007

Sato, R., Suzuki, T., Katayose, Y., Miura, K., Shiiba, K., Miki, Y., et al. (2012). Aromatase in colon carcinoma. Anticancer Res. 32, 3069-3075.

Sato, R., Suzuki, T., Katayose, Y., Miura, K., Shiiba, K., Tateno, H., et al. (2009). Steroid sulfatase and estrogen sulfotransferase in colon carcinoma: regulators of intratumoral estrogen concentrations and potent prognostic factors. Cancer Res. 69, 914-922. doi: 10.1158/0008-5472.CAN-08-0906

Schweikert, H. U., Wolf, L., and Romalo, G. (1995). Oestrogen formation from androstenedione in human bone. Clin. Endocrinol. (Oxf). 43, 37-42. doi: 10.1111/j.1365-2265.1995.tb01890.x

Scublinsky, A., Marin, C., and Gurpide, E. (1976). Localization of estradiol $17 \beta$ dehydrogenase in human endometrium. J. Steroid Biochem. 7, 745-747. doi: 10.1016/0022-4731(76)90174-6

Segawa, T., Shozu, M., Murakami, K., Kasai, T., Shinohara, K., Nomura, K., et al. (2005). Aromatase expression in stromal cells of endometrioid endometrial cancer correlates with poor survival. Clin. Cancer Res. 11, 2188-2194. doi: 10.1158/1078-0432.CCR-04-1859

Selcer, K. W., and Difrancesca, H. M. (2012). Characterization of steroid sulfatase in the MC3T3-E1 mouse pre-osteoblastic cell line. Steroids 77, 696-702. doi: 10.1016/j.steroids.2012.02.024

Setiawan, V. W., Doherty, J. A., Shu, X. O., Akbari, M. R., Chen, C., De Vivo, I., et al. (2009). Two estrogen-related variants in $\mathrm{CYP}_{19} \mathrm{~A}_{1}$ and endometrial cancer risk: a pooled analysis in the epidemiology of endometrial cancer consortium. Cancer Epidemiol. Biomarkers Prev. 18, 242-247. doi: 10.1158/1055-9965.EPI-08-0689

Setiawan, V. W., Hankinson, S. E., Colditz, G. A., Hunter, D. J., and De Vivo, I. (2004). HSD17B1 gene polymorphisms and risk of endometrial and breast cancer. Cancer Epidemiol. Biomarkers Prev. 13, 213-219. doi: 10.1158/1055-9965.EPI-03-0241

Shang, E., Lai, K., Packer, A. I., Paik, J., Blaner, W. S., de Morais Vieira, M., et al. (2002). Targeted disruption of the mouse cis-retinol dehydrogenase gene: visual and nonvisual functions. J. Lipid Res. 43, 590-597.

Shehu, A., Mao, J., Gibori, G. B., Halperin, J., Le, J., Devi, Y. S., et al. (2008). Prolactin receptor-associated protein $/ 17 \beta$-hydroxysteroid dehydrogenase type 7 gene (Hsd17b7) plays a crucial role in embryonic development and fetal survival. Mol. Endocrinol. 22, 2268-2277. doi: 10.1210/me.2008-0165

Shen, Z., Peng, Z., Sun, Y., Vaananen, H. K., and Poutanen, M. (2008). Overexpression of human hydroxysteroid (17 $\beta$ ) dehydrogenase 2 induces disturbance in skeletal development in young male mice. J. Bone Miner. Res. 23, 1217-1226. doi: 10.1359/jbmr.080322

Shi, L., Yang, X., Dong, X., and Zhang, B. (2016). Polymorphism of HSD17B1 Ser312Gly with Cancer Risk: Evidence from 66,147 Subjects. Twin Res. Hum. Genet. 19, 136-145. doi: 10.1017/thg.2016.6

Shim, G. J., Warner, M., Kim, H. J., Andersson, S., Liu, L., Ekman, J., et al. (2004). Aromatase-deficient mice spontaneously develop a lymphoproliferative autoimmune disease resembling Sjogren's syndrome. Proc. Natl. Acad. Sci. U.S.A. 101, 12628-12633. doi: 10.1073/pnas.0405 099101

Shimizu, K. (1979). Metabolism of $\left[17-{ }^{2} \mathrm{H}\right]$ pregnenolone into $5-\left[17 \beta-{ }^{2} \mathrm{H}, 17 \alpha-\right.$ ${ }^{18} \mathrm{O}$ ] androstene- $3 \beta, 17 \alpha$-diol and other products by incubation with the microsomal fraction of boar testis under ${ }^{18} \mathrm{O}_{2}$ atmosphere. Biochim. Biophys. Acta 575, 37-45. doi: 10.1016/0005-2760(79)90128-0

Shimodaira, M., Nakayama, T., Sato, I., Sato, N., Izawa, N., Mizutani, Y., et al. (2012). Estrogen synthesis genes $\mathrm{CYP}_{19} \mathrm{~A}_{1}, \mathrm{HSD}_{3} \mathrm{~B}_{1}$, and
$\mathrm{HSD}_{3} \mathrm{~B}_{2}$ in hypertensive disorders of pregnancy. Endocrine 42, 700-707. doi: 10.1007/s12020-012-9699-7

Shimodaira, M., Nakayama, T., Sato, N., Aoi, N., Sato, M., Izumi, Y., et al. (2010). Association of HSD3B1 and HSD3B2 gene polymorphisms with essential hypertension, aldosterone level, and left ventricular structure. Eur. J. Endocrinol. 163, 671-680. doi: 10.1530/EJE-10-0428

Siegfried, J. M., and Stabile, L. P. (2014). Estrongenic steroid hormones in lung cancer. Semin. Oncol. 41, 5-16. doi: 10.1053/j.seminoncol.2013.12.009

Simard, J., Ricketts, M. L., Gingras, S., Soucy, P., Feltus, F. A., and Melner, M. H. (2005). Molecular biology of the $3 \beta$-hydroxysteroid dehydrogenase $/ \Delta^{5}-\Delta^{4}$ isomerase gene family. Endocr. Rev. 26, 525-582. doi: 10.1210/er.2002-0050

Simard, M., Plante, J., Boucher, M., Provost, P. R., and Tremblay, Y. (2010). Type 2 and 5,17 $\beta$-hydroxysteroid dehydrogenases and androgen receptor in human fetal lungs. Mol. Cell. Endocrinol. 319, 79-87. doi: 10.1016/j.mce.2009. 12.007

Simoens, S., Dunselman, G., Dirksen, C., Hummelshoj, L., Bokor, A., Brandes, I., et al. (2012). The burden of endometriosis: costs and quality of life of women with endometriosis and treated in referral centres. Hum. Reprod. 27, 1292-1299. doi: 10.1093/humrep/des073

Simoens, S., Hummelshoj, L., Dunselman, G., Brandes, I., Dirksen, C., and D'Hooghe, T. (2011). Endometriosis cost assessment (the EndoCost study): a cost-of-illness study protocol. Gynecol. Obstet. Invest. 71, 170-176. doi: $10.1159 / 000316055$

Singh, M. M., Trivedi, R. N., Chauhan, S. C., Srivastava, V. M., Makker, A., Chowdhury, S. R., et al. (1996). Uterine estradiol and progesterone receptor concentration, activities of certain antioxidant enzymes and dehydrogenases and histoarchitecture in relation to time of secretion of nidatory estrogen and high endometrial sensitivity in rat. J. Steroid Biochem. Mol. Biol. 59, 215-224. doi: 10.1016/S0960-0760(96)00102-1

Singhal, N., Vatandoust, S., and Brown, M. P. (2015). Phase II study evaluating efficacy and safety of everolimus with letrozole for management of advanced (unresectable or metastatic) non-small cell lung cancer after failure of platinum-based treatment: a preliminary analysis of toxicity. Cancer Chemother. Pharmacol. 75, 325-331. doi: 10.1007/s00280-014-2644-0

Sinreih, M., Anko, M., Zukunft, S., Adamski, J., and Rizner, T. L. (2014). Important roles of the AKR1C2 and SRD5A1 enzymes in progesterone metabolism in endometrial cancer model cell lines. Chem. Biol. Interact. 234, 297-308. doi: 10.1016/j.cbi.2014.11.012.

Sinreih, M., Hevir, N., and Rizner, T. L. (2013). Altered expression of genes involved in progesterone biosynthesis, metabolism and action in endometrial cancer. Chem. Biol. Interact. 202, 210-217. doi: 10.1016/j.cbi.2012.11.012

Sinreih, M., Knific, T., Anko, M., Hevir, N., Vouk, K., Jerin, A., et al. (2017a). The significance of the sulfatase pathway for local estrogen formation in endometrial cancer. Front. Pharmacol. 8:368. doi: 10.3389/fphar.2017.00368

Sinreih, M., Stupar, S., Cemazar, L., Verdenik, I., Frkovic Grazio, S., Smrkolj, S., et al. (2017b). STAR and AKR1B10 are down-regulated in high-grade endometrial cancer. J. Steroid Biochem. Mol. Biol.. doi: 10.1016/j.jsbmb.2017.02.015

Sivik, T. (2012). Elucidating the Role of $17 \beta$ Hydroxysteroid Dehydrogenase Type 14 in Normal Physiology and in Breast Cancer. Department of Clinical and Experimental Medicine: Linköping University (Linköping).

Skjefstad, K., Grindstad, T., Khanehkenari, M. R., Richardsen, E., Donnem, T., Kilvaer, T., et al. (2016). Prognostic relevance of estrogen receptor $\alpha, \beta$ and aromatase expression in non-small cell lung cancer. Steroids 113, 5-13. doi: 10.1016/j.steroids.2016.05.008

Slominski, A., Zbytek, B., Nikolakis, G., Manna, P. R., Skobowiat, C. Zmijewski, M., et al. (2013). Steroidogenesis in the skin: implications for local immune functions. J. Steroid Biochem. Mol. Biol. 137, 107-123. doi: 10.1016/j.jsbmb.2013.02.006

Slomovitz, B. M., Jiang, Y., Yates, M. S., Soliman, P. T., Johnston, T., Nowakowski, M., et al. (2015). Phase II study of everolimus and letrozole in patients with recurrent endometrial carcinoma. J. Clin. Oncol. 33, 930-936. doi: 10.1200/JCO.2014.58.3401

Smith, A., Contreras, C., Ko, K. H., Chow, J., Dong, X., Tuo, B., et al. (2008). Gender-specific protection of estrogen against gastric acid-induced duodenal injury: stimulation of duodenal mucosal bicarbonate secretion. Endocrinology 149, 4554-4566. doi: 10.1210/en.2007-1597 
Smith, E. P., Boyd, J., Frank, G. R., Takahashi, H., Cohen, R. M., Specker, B., et al. (1994). Estrogen resistance caused by a mutation in the estrogen-receptor gene in a man. N. Engl. J. Med. 331, 1056-1061. doi: 10.1056/NEJM199410203311604

Smuc, T., Hevir, N., Ribic-Pucelj, M., Husen, B., Thole, H., and Rizner, T. L. (2009). Disturbed estrogen and progesterone action in ovarian endometriosis. Mol. Cell. Endocrinol. 301, 59-64. doi: 10.1016/j.mce.2008.07.020

Smuc, T., Pucelj, M. R., Sinkovec, J., Husen, B., Thole, H., and Lanisnik Rizner, T. (2007). Expression analysis of the genes involved in estradiol and progesterone action in human ovarian endometriosis. Gynecol. Endocrinol. 23, 105-111. doi: 10.1080/09513590601152219

Smuc, T., and Rizner, T. L. (2009). Aberrant pre-receptor regulation of estrogen and progesterone action in endometrial cancer. Mol. Cell. Endocrinol. 301, 74-82. doi: 10.1016/j.mce.2008.09.019

Smuc, T., Rupreht, R., Sinkovec, J., Adamski, J., and Rizner, T. L. (2006). Expression analysis of estrogen-metabolizing enzymes in human endometrial cancer. Mol. Cell. Endocrinol. 248, 114-117. doi: 10.1016/j.mce.2005.10.013

Soma, K. K., Rendon, N. M., Boonstra, R., Albers, H. E., and Demas, G. E. (2015). DHEA effects on brain and behavior: insights from comparative studies of aggression. J. Steroid Biochem. Mol. Biol. 145, 261-272. doi: 10.1016/j.jsbmb.2014.05.011

Soubhye, J., Alard, I. C., van Antwerpen, P., and Dufrasne, F. (2015). Type 2, 17-beta hydroxysteroid dehydrogenase as a novel target for the treatment of osteoporosis. Future Med. Chem. 7, 1431-1456. doi: 10.4155/fmc.15.74

Steckelbroeck, S., Jin, Y., Gopishetty, S., Oyesanmi, B., and Penning, T. M. (2004a). Human cytosolic 3alpha-hydroxysteroid dehydrogenases of the aldo-keto reductase superfamily display significant 3 beta-hydroxysteroid dehydrogenase activity: implications for steroid hormone metabolism and action. J. Biol. Chem. 279, 10784-10795. doi: 10.1074/jbc.M313308200

Steckelbroeck, S., Lutjohann, D., Bauman, D. R., Ludwig, M., Friedl, A., Hans, V. H., et al. (2010). Non-stereo-selective cytosolic human brain tissue 3ketosteroid reductase is refractory to inhibition by AKR1C inhibitors. Biochim. Biophys. Act. 1801, 1221-1231. doi: 10.1016/j.bbalip.2010.07.005

Steckelbroeck, S., Nassen, A., Ugele, B., Ludwig, M., Watzka, M., Reissinger, A., et al. (2004b). Steroid sulfatase (STS) expression in the human temporal lobe: enzyme activity, mRNA expression and immunohistochemistry study. J. Neurochem. 89, 403-417. doi: 10.1046/j.1471-4159.2004.02336.x

Steckelbroeck, S., Stoffel-Wagner, B., Reichelt, R., Schramm, J., Bidlingmaier, F., Siekmann, L., et al. (1999). Characterization of 17ß-hydroxysteroid dehydrogenase activity in brain tissue: testosterone formation in the human temporal lobe. J. Neuroendocrinol. 11, 457-464. doi: $10.1046 /$ j.1365-2826.1999.00363.x

Steckelbroeck, S., Watzka, M., Reichelt, R., Hans, V. H., Stoffel-Wagner, B., Heidrich, D. D., et al. (2001). Characterization of the $5 \alpha$-reductase- $3 \alpha-$ hydroxysteroid dehydrogenase complex in the human brain. J. Clin. Endocrinol. Metab. 86, 1324-1331. doi: 10.1210/jcem.86.3.7325

Steckelbroeck, S., Watzka, M., Reissinger, A., Wegener-Toper, P., Bidlingmaier, F., Bliesener, N., et al. (2003). Characterisation of estrogenic 17 $\beta$-hydroxysteroid dehydrogenase (17 $\beta$-HSD) activity in the human brain. J. Steroid Biochem. Mol. Biol. 86, 79-92. doi: 10.1016/S0960-0760(03)00251-6

Stelzer, G., Rosen, N., Plaschkes, I., Zimmerman, S., Twik, M., Fishilevich, S., et al. (2016). The GeneCards suite: from gene data mining to disease genome sequence analyses. Curr Protoc Bioinformatics 54, 1.30, 31-31, 30, 33. doi: $10.1002 /$ cpbi.5

Stoffel-Wagner, B. (2001). Neurosteroid metabolism in the human brain. Eur. J. Endocrinol. 145, 669-679. doi: 10.1530/eje.0.1450669

Stoffel-Wagner, B., Beyenburg, S., Watzka, M., Blumcke, I., Bauer, J., Schramm, J., et al. (2000). Expression of $5 \alpha$-reductase and $3 \alpha$-hydroxisteroid oxidoreductase in the hippocampus of patients with chronic temporal lobe epilepsy. Epilepsia 41, 140-147. doi: 10.1111/j.1528-1157.2000.tb00133.x

Stoffel-Wagner, B., Watzka, M., Schramm, J., Bidlingmaier, F., and Klingmuller, D. (1999a). Expression of CYP19 (aromatase) mRNA in different areas of the human brain. J. Steroid Biochem. Mol. Biol. 70, 237-241. doi: 10.1016/S0960-0760(99)00114-4

Stoffel-Wagner, B., Watzka, M., Steckelbroeck, S., Ludwig, M., Clusmann, H., Bidlingmaier, F., et al. (2003). Allopregnanolone serum levels and expression of 5 alpha-reductase and 3 alpha-hydroxysteroid dehydrogenase isoforms in hippocampal and temporal cortex of patients with epilepsy. Epilepsy Res. 54, 11-19. doi: 10.1016/S0920-1211(03)00036-6
Stoffel-Wagner, B., Watzka, M., Steckelbroeck, S., Schramm, J., Bidlingmaier, J. F., and Klingmuller, D. (1999b). Expression of 17 $\beta$-hydroxysteroid dehydrogenase types 1, 2, 3 and 4 in the human temporal lobe. J. Endocrinol. 160, 119-126. doi: $10.1677 /$ joe. 0.1600119

Stoffel-Wagner, B., Watzka, M., Steckelbroeck, S., Schwaab, R., Schramm, J., Bidlingmaier, F., et al. (1998a). Expression of CYP19 (aromatase) mRNA in the human temporal lobe. Biochem. Biophys. Res. Commun. 244, 768-771. doi: 10.1006/bbrc. 1998.8337

Stoffel-Wagner, B., Watzka, M., Steckelbroeck, S., Wickert, L., Schramm, J., Romalo, G., et al. (1998b). Expression of $5 \alpha$-reductase in the human temporal lobe of children and adults. J. Clin. Endocrinol. Metab. 83, 3636-3642.

Strott, C. A. (2002). Sulfonation and molecular action. Endocr. Rev. 23, 703-732. doi: 10.1210/er.2001-0040

Sudeshna, T., Anand, K., and Medhamurthy, R. (2013). Analysis of $20 \alpha-$ hydroxysteroid dehydrogenase expression in the corpus luteum of the buffalo cow: effect of prostaglandin F2- $\alpha$ treatment on circulating $20 \alpha$-hydroxyprogesterone levels. Reprod. Biol. Endocrinol. 11:111. doi: 10.1186/1477-7827-11-111

Sun, X. F., Ahmadi, A., Arbman, G., Wallin, A., Asklid, D., and Zhang, H. (2005). Polymorphisms in sulfotransferase $1 \mathrm{~A} 1$ and glutathione Stransferase P1 genes in relation to colorectal cancer risk and patients' survival. World J. Gastroenterol. 11, 6875-6879. doi: 10.3748/wjg.v11.i4 3.6875

Svoboda, M., Hamilton, G., and Thalhammer, T. (2010). Steroid hormone metabolizing enzymes in benign and malignant human bone tumors. Expert Opin. Drug Metab. Toxicol. 6, 427-437. doi: 10.1517/174252510035 92129

Svoboda, M., Thalhammer, T., Aust, S., Arrich, F., Assadian, O., and Toma, C. D. (2007). Estrogen sulfotransferase (SULT1E1) expression in benign and malignant human bone tumors. J. Surg. Oncol. 95, 572-581. doi: $10.1002 /$ jso. 20748

Syed, F., and Khosla, S. (2005). Mechanisms of sex steroid effects on bone. Biochem. Biophys. Res. Commun. 328, 688-696. doi: 10.1016/j.bbrc.2004. 11.097

Taga, S., Yoshida, N., and Sekiba, K. (1990). Distribution and cyclic change of aromatase cytochrome P-450 activity in human uteri. J. Steroid Biochem. Mol. Biol. 37, 741-745. doi: 10.1016/0960-0760(90)9 0359-S

Takeyama, J., Suzuki, T., Hirasawa, G., Muramatsu, Y., Nagura, H., Iinuma, K., et al. (2000). 17ß-hydroxysteroid dehydrogenase type 1 and 2 expression in the human fetus. J. Clin. Endocrinol. Metab. 85, 410-416. doi: $10.1210 /$ jcem.85.1.6323

Tanaka, K., Kubushiro, K., Iwamori, Y., Okairi, Y., Kiguchi, K., Ishiwata, I., et al. (2003). Estrogen sulfotransferase and sulfatase: Roles in the regulation of estrogen activity in human uterine endometrial carcinomas. Cancer Sci. 94, 871-876. doi: 10.1111/j.1349-7006.2003.tb 01369.x

Tanaka, K., Shimizu, K., Kakegawa, S., Ohtaki, Y., Nagashima, T., Kaira, K., et al. (2016). Prognostic significance of aromatase and estrogen receptor beta expression in EGFR wild-type lung adenocarcinoma. Am. J. Transl. Res. 8, 81-97.

Tanaka, S., Miki, Y., Hashimoto, C., Takagi, K., Doe, Z., Li, B., et al. (2015). The role of $5 \alpha$-reductase type 1 associated with intratumoral dihydrotestosterone concentrations in human endometrial carcinoma. Mol. Cell. Endocrinol. 401, 56-64. doi: 10.1016/j.mce.2014.11.022

Tang, T., Li, L., Tang, J., Li, Y., Lin, W. Y., Martin, F., et al. (2010). A mouse knockout library for secreted and transmembrane proteins. Nat. Biotechnol. 28, 749-755. doi: 10.1038/nbt.1644

Tangen, I. L., Onyango, T. B., Kopperud, R., Berg, A., Halle, M. K., Oyan, A. M., et al. (2016). Androgen receptor as potential therapeutic target in metastatic endometrial cancer. Oncotarget 7, 49289-49298. doi: 10.18632/oncotarget.10334

Tangen, I. L., Werner, H. M., Berg, A., Halle, M. K., Kusonmano, K., Trovik, J., et al. (2014). Loss of progesterone receptor links to high proliferation and increases from primary to metastatic endometrial cancer lesions. Eur J Cancer 50, 3003-3010. doi: 10.1016/j.ejca.2014.09.003

Taniuchi, S., Fujishima, F., Miki, Y., Abe, K., Nakamura, Y., Sato, S., et al. (2014). Tissue concentrations of estrogens and aromatase immunolocalization 
in interstitial pneumonia of human lung. Mol. Cell. Endocrinol. 392, 136-143. doi: 10.1016/j.mce.2014.05.016

Tashiro, A., Sasano, H., Nishikawa, T., Yabuki, N., Muramatsu, Y., Coughtrie, M. W., et al. (2000). Expression and activity of dehydroepiandrosterone sulfotransferase in human gastric mucosa. J. Steroid Biochem. Mol. Biol. 72, 149-154. doi: 10.1016/S0960-0760(00)00020-0

Taveira-DaSilva, A. M., and Moss, J. (2014). Management of lymphangioleiomyomatosis. F1000Prime Rep. 6, 116. doi: 10.12703/P6-116

Terry, K., McGrath, M., Lee, I. M., Buring, J., and De Vivo, I. (2010). Genetic variation in CYP11A1 and StAR in relation to endometrial cancer risk. Gynecol. Oncol. 117, 255-259. doi: 10.1016/j.ygyno.2010.02.002

Teubner, W., Meinl, W., Florian, S., Kretzschmar, M., and Glatt, H. (2007). Identification and localization of soluble sulfotransferases in the human gastrointestinal tract. Biochem. J. 404, 207-215. doi: 10.1042/BJ20 061431

Thiboutot, D., Martin, P., Volikos, L., and Gilliland, K. (1998). Oxidative activity of the type 2 isozyme of $17 \beta$-hydroxysteroid dehydrogenase (17 $\beta$-HSD) predominates in human sebaceous glands. J. Invest. Dermatol. 111, 390-395. doi: 10.1046/j.1523-1747.1998.00322.x

Thompson, D. J., O’Mara, T. A., Glubb, D. M., Painter, J. N., Cheng, T., Folkerd, E., et al. (2016). $\mathrm{CYP}_{19} \mathrm{~A}_{1}$ fine-mapping and Mendelian randomization: estradiol is causal for endometrial cancer. Endocr. Relat. Cancer 23, 77-91. doi: 10.1530/ERC-15-0386

Tong, M. H., Jiang, H., Liu, P., Lawson, J. A., Brass, L. F., and Song, W. C. (2005). Spontaneous fetal loss caused by placental thrombosis in estrogen sulfotransferase-deficient mice. Nat. Med. 11, 153-159. doi: 10.1038/nm1184

Törn, S., Nokelainen, P., Kurkela, R., Pulkka, A., Menjivar, M., Ghosh, S., et al. (2003). Production, purification, and functional analysis of recombinant human and mouse 17 $\beta$-hydroxysteroid dehydrogenase type 7. Biochem. Biophys. Res. Commun. 305, 37-45. doi: 10.1016/S0006-291X(03)00694-6

Townsend, E. A., Miller, V. M., and Prakash, Y. S. (2012). Sex differences and sex steroids in lung health and disease. Endocr. Rev. 33, 1-47. doi: 10.1210/er.2010-0031

Townsend, E. A., Thompson, M. A., Pabelick, C. M., and Prakash, Y. S. (2010). Rapid effects of estrogen on intracellular $\mathrm{Ca}^{2+}$ regulation in human airway smooth muscle. Am. J. Physiol. Lung Cell. Mol. Physiol. 298 L521-530. doi: 10.1152/ajplung.00287.2009

Tsai, S. J., Wu, M. H., Lin, C. C., Sun, H. S., and Chen, H. M. (2001). Regulation of steroidogenic acute regulatory protein expression and progesterone production in endometriotic stromal cells. J. Clin. Endocrinol. Metab. 86, 5765-5773. doi: 10.1210/jcem.86.12.8082

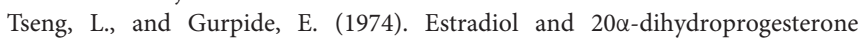
dehydrogenase activities in human endometrium during the menstrual cycle. Endocrinology 94, 419-423. doi: 10.1210/endo-94-2-419

Tseng, L., Gusberg, S. B., and Gurpide, E. (1977). Estradiol receptor and 17 betadehydrogenase in normal and abnormal human endometrium. Ann. N. Y. Acad. Sci. 286, 190-198. doi: 10.1111/j.1749-6632.1977.tb29416.x

Tseng, L., and Mazella, J. (2002). Endometrial cell specific gene activation during implantation and early pregnancy. Front. Biosci. 7, d1566-d1574. doi: $10.2741 / \mathrm{A} 861$

Tseng, L., Mazella, J., Mann, W. J., and Chumas, J. (1982). Estrogen synthesis in normal and malignant human endometrium. J. Clin. Endocrinol. Metab. 55, 1029-1031. doi: 10.1210/jcem-55-5-1029

Tsuchiya, M., Nakao, H., Katoh, T., Sasaki, H., Hiroshima, M., Tanaka, T., et al. (2005). Association between endometriosis and genetic polymorphisms of the estradiol-synthesizing enzyme genes HSD17B1 and CYP19. Hum. Reprod. 20, 974-978. doi: 10.1093/humrep/deh726

Tuo, B., Wen, G., Wei, J., Liu, X., Wang, X., Zhang, Y., et al. (2011). Estrogen regulation of duodenal bicarbonate secretion and sex-specific protection of human duodenum. Gastroenterology 141, 854-863. doi: 10.1053/j.gastro.2011.05.044

Utsunomiya, H., Ito, K., Suzuki, T., Kitamura, T., Kaneko, C., Nakata, T., et al. (2004). Steroid sulfatase and estrogen sulfotransferase in human endometrial carcinoma. Clin. Cancer Res. 10, 5850-5856. doi: 10.1158/1078-0432.CCR-04-0040

Utsunomiya, H., Suzuki, T., Ito, K., Moriya, T., Konno, R., Sato, S., et al. (2003). The correlation between the response to progestogen treatment and the expression of progesterone receptor B and $17 \beta$-hydroxysteroid dehydrogenase type 2 in human endometrial carcinoma. Clin. Endocrinol. (Oxf). 58, 696-703. doi: 10.1046/j.1365-2265.2003.01766.x

Utsunomiya, H., Suzuki, T., Kaneko, C., Takeyama, J., Nakamura, J., Kimura, K., et al. (2001). The analyses of $17 \beta$-hydroxysteroid dehydrogenase isozymes in human endometrial hyperplasia and carcinoma. J. Clin. Endocrinol. Metab. 86, 3436-3443. doi: 10.1210/jcem.86.7.7661

van der Eerden, B. C., Lowik, C. W., Wit, J. M., and Karperien, M. (2004). Expression of estrogen receptors and enzymes involved in sex steroid metabolism in the rat tibia during sexual maturation. J. Endocrinol. 180, 457-467. doi: 10.1677/joe.0.1800457

Vanderschueren, D., Gaytant, J., Boonen, S., and Venken, K. (2008). Androgens and bone. Curr. Opin. Endocrinol. Diabetes Obes. 15, 250-254 doi: 10.1097/MED.0b013e3282fe6ca9

Vani, S., McDonald, S. E., Williams, A. R., Mason, J. I., Thong, K. J., and Critchley, H. O. (2007). Mid-luteal endometrial intracrinology following controlled ovarian hyperstimulation involving use of a gonadotrophin releasing hormone antagonist. Hum. Reprod. 22, 2981-2991. doi: 10.1093/humrep/dem269

Varlamov, O., Bethea, C. L., and Roberts, C. T. Jr. (2014). Sex-specific differences in lipid and glucose metabolism. Front. Endocrinol. (Lausanne). 5:241. doi: 10.3389/fendo.2014.00241

Vasquez, Y. M., and DeMayo, F. J. (2013). Role of nuclear receptors in blastocyst implantation. Semin. Cell Dev. Biol. 24, 724-735. doi: 10.1016/j.semcdb.2013.08.004

Velasco, I., Rueda, J., and Acien, P. (2006). Aromatase expression in endometriotic tissues and cell cultures of patients with endometriosis. Mol. Hum. Reprod. 12, 377-381. doi: 10.1093/molehr/gal041

Vercellini, P., Vigano, P., Somigliana, E., and Fedele, L. (2014). Endometriosis: pathogenesis and treatment. Nat. Rev. Endocrinol. 10, 261-275. doi: 10.1038/nrendo.2013.255

Verma, M. K., Miki, Y., Abe, K., Suzuki, T., Niikawa, H., Suzuki, S., et al. (2013). Intratumoral localization and activity of $17 \beta$-hydroxysteroid dehydrogenase type 1 in non-small cell lung cancer: a potent prognostic factor. J. Transl. Med. 11, 167. doi: 10.1186/1479-5876-11-167

Verma, M. K., Miki, Y., and Sasano, H. (2011). Aromatase in human lung carcinoma. Steroids 76, 759-764. doi: 10.1016/j.steroids.2011.02.020

Vidal, O., Lindberg, M. K., Hollberg, K., Baylink, D. J., Andersson, G., Lubahn, D. B., et al. (2000). Estrogen receptor specificity in the regulation of skeletal growth and maturation in male mice. Proc. Natl. Acad. Sci. U.S.A. 97, 5474-5479. doi: 10.1073 /pnas.97.10.5474

Vouk, K., Smuc, T., Guggenberger, C., Ribic-Pucelj, M., Sinkovec, J., Husen, B., et al. (2011). Novel estrogen-related genes and potential biomarkers of ovarian endometriosis identified by differential expression analysis. J. Steroid Biochem. Mol. Biol. 125, 231-242. doi: 10.1016/j.jsbmb.2011.03.010

Walker, V. R., and Korach, K. S. (2004). Estrogen receptor knockout mice as a model for endocrine research. ILAR J. 45, 455-461. doi: 10.1093/ilar.45.4.455

Wang, F., Vihma, V., Soronen, J., Turpeinen, U., Hamalainen, E., SavolainenPeltonen, H., et al. (2013). 17 $\beta$-Estradiol and estradiol fatty acyl esters and estrogen-converting enzyme expression in adipose tissue in obese men and women. J. Clin. Endocrinol. Metab. 98, 4923-4931. doi: 10.1210/jc.2013-2605

Wang, H., and Dey, S. K. (2006). Roadmap to embryo implantation: clues from mouse models. Nat. Rev. Genet. 7, 185-199. doi: 10.1038/n rg1808

Wang, X. Q., Aka, J. A., Li, T., Xu, D., Doillon, C. J., and Lin, S. X. (2017). Inhibition of $17 \beta$-hydroxysteroid dehydrogenase type 7 modulates breast cancer protein profile and enhances apoptosis by down-regulating GRP78. J. Steroid Biochem. Mol. Biol. 172, 188-197. doi: 10.1016/j.jsbmb.2017. 06.009

Warren, J. C., and French, A. P. (1965). DISTRIBUTION OF STEROID SULFATASE IN HUMAN TISSUES. J. Clin. Endocrinol. Metab. 25, 278-282. doi: 10.1210/jcem-25-2-278

Watanabe, K., Sasano, H., Harada, N., Ozaki, M., Niikura, H., Sato, S., et al. (1995). Aromatase in human endometrial carcinoma and hyperplasia. Immunohistochemical, in situ hybridization, and biochemical studies. Am. J. Pathol. 146, 491-500.

Watzka, M., Bidlingmaier, F., Schramm, J., Klingmuller, D., and StoffelWagner, B. (1999). Sex- and age-specific differences in human brain CYP11A1 mRNA expression. J. Neuroendocrinol. 11, 901-905. doi: 10.1046/j.1365-2826.1999.00407.x 
Wishart, D. S., Feunang, Y. D., Guo, A. C., Lo, E. J., Marcu, A., Grant, J. R., et al. (2018). DrugBank 5.0: a major update to the DrugBank database for 2018. Nucleic Acids Res. 46, D1074-D1082. doi: 10.1093/nar/gkx1037

Wishart, D. S., Jewison, T., Guo, A. C., Wilson, M., Knox, C., Liu, Y., et al. (2013). HMDB 3.0-the human metabolome database in 2013. Nucleic Acids Res. 41, D801-D807. doi: 10.1093/nar/gks1065

Wöhrle, D., Barbi, G., Schulz, W., and Steinbach, P. (1990). Heterozygous expression of X-linked chondrodysplasia punctata. Complex chromosome aberration including deletion of MIC2 and STS. Hum. Genet. 86, 215-218. doi: 10.1007/BF00197708

Woo, L. W., Purohit, A., and Potter, B. V. (2011). Development of steroid sulfatase inhibitors. Mol. Cell. Endocrinol. 340, 175-185. doi: 10.1016/j.mce.2010.12.035

Wu, H. C., Tuo, B. G., Wu, W. M., Gao, Y., Xu, Q. Q., and Zhao, K. (2008). Prevalence of peptic ulcer in dyspeptic patients and the influence of age, sex, and Helicobacter pylori infection. Dig. Dis. Sci. 53, 2650-2656. doi: 10.1007/s10620-007-0177-7

Xiao, J., Zheng, Y., Zhou, Y., Zhang, P., Wang, J., Shen, F., et al. (2014). Sulfotransferase SULT1A1 Arg213His polymorphism with cancer risk: a meta-analysis of 53 case-control studies. PLOS ONE 9:e106774. doi: 10.1371/journal.pone.0106774

Yague, J. G., Azcoitia, I., DeFelipe, J., Garcia-Segura, L. M., and Munoz, A. (2010). Aromatase expression in the normal and epileptic human hippocampus. Brain Res. 1315, 41-52. doi: 10.1016/j.brainres.2009.09.111

Yague, J. G., Munoz, A., de Monasterio-Schrader, P., Defelipe, J., Garcia-Segura, L. M., and Azcoitia, I. (2006). Aromatase expression in the human temporal cortex. Neuroscience 138, 389-401. doi: 10.1016/j.neuroscience.2005.11.054

Yamaki, J., Yamamoto, T., and Okada, H. (1985). Aromatization of androstenedione by normal and neoplastic endometrium of the uterus. J. Steroid Biochem. 22, 63-66. doi: 10.1016/0022-4731(85)90142-6

Yamamoto, T., Fukuoka, M., Fujimoto, Y., Kitawaki, J., Nakakoshi, M., Yoshihama, M., et al. (1990a). Inhibitory effect of a new androstenedione derivative, $14 \alpha$-hydroxy-4-androstene-3,6,17-trione $(14 \alpha$-OHAT) on aromatase activity of human uterine tumors. J. Steroid Biochem. 36, 517-21. doi: 10.1016/0022-4731(90)90167-Q

Yamamoto, T., Kitawaki, J., Urabe, M., Honjo, H., Tamura, T., Noguchi, T., et al. (1993a). Estrogen productivity of endometrium and endometrial cancer tissue; influence of aromatase on proliferation of endometrial cancer cells. J. Steroid Biochem. Mol. Biol. 44, 463-468. doi: 10.1016/0960-0760(93)90251-Q

Yamamoto, T., Noguchi, T., Tamura, T., Kitawaki, J., and Okada, H. (1993b). Evidence for estrogen synthesis in adenomyotic tissues. Am. J. Obstet. Gynecol. 169, 734-738. doi: 10.1016/0002-9378(93)90654-2

Yamamoto, T., Urabe, M., Naitoh, K., Kitawaki, J., Honjo, H., and Okada, H. (1990b). Estrone sulfatase activity in human uterine leiomyoma. Gynecol. Oncol. 37, 315-318. doi: 10.1016/0090-8258(90)90358-R

Yanaihara, Yanaihara, T., Toma, Y., Shimizu, Y., Saito, H., Okai, T., et al. (2001). Localization and expression of steroid sulfatase in human fallopian tubes. Steroids 66, 87-91. doi: 10.1016/S0039-128X(00)00204-X

Yang, W., Wu, G., Broeckel, U., Smith, C. A., Turner, V., Haidar, C. E., et al. (2016). Comparison of genome sequencing and clinical genotyping for pharmacogenes. Clin. Pharmacol. Ther. 100, 380-388. doi: 10.1002/cpt.411

Yang, X. Y., Wu, W. J., Yang, C., Yang, T., He, J. D., Yang, Z., et al. (2013). Association of HSD17B3 and HSD3B1 polymorphisms with acne vulgaris in Southwestern Han Chinese. Dermatology 227, 202-208. doi: 10.1159/000353581

Ye, X. Y., Chen, S. Y., Wu, S., Yoon, D. S., Wang, H., Hong, Z., et al. (2017). Discovery of Clinical Candidate 2-((2S,6S)-2-Phenyl-6-hydroxyadamantan-2yl)-1-(3'-hydroxyazetidin-1-yl)ethanone [BMS-816336], an orally active novel selective $11 \beta$-Hydroxysteroid dehydrogenase type 1 inhibitor. J. Med. Chem. 60, 4932-4948. doi: 10.1021/acs.jmedchem.7b00211

Yu, L., Romero, D. G., Gomez-Sanchez, C. E., and Gomez-Sanchez, E. P. (2002). Steroidogenic enzyme gene expression in the human brain. Mol. Cell. Endocrinol. 190, 9-17. doi: 10.1016/S0303-7207(02)00041-2

Yuchi, Y., Cai, Y., Legein, B., De Groef, S., Leuckx, G., Coppens, V., et al. (2015). Estrogen receptor $\alpha$ regulates $\beta$-cell formation during pancreas development and following injury. Diabetes 64, 3218-3228. doi: 10.2337/ db14-1798

Zacher, A., Kaulich, K., Stepanow, S., Wolter, M., Kohrer, K., Felsberg, J., et al. (2016). Molecular diagnostics of gliomas using next generation sequencing of a glioma-tailored gene panel. Brain Pathol. 27, 146-159. doi: 10.1111/ bpa. 12367

Zakharov, V., Lin, H. K., Azzarello, J., McMeekin, S., Moore, K. N., Penning, T. M.,

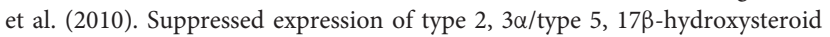
dehydrogenase (AKR1C3) in endometrial hyperplasia and carcinoma. Int. J. Clin. Exp. Pathol. 3, 608-617.

Zarrabeitia, M. T., Hernandez, J. L., Valero, C., Zarrabeitia, A., Amado, J. A., Gonzalez-Macias, J., et al. (2007). Adiposity, estradiol, and genetic variants of steroid-metabolizing enzymes as determinants of bone mineral density. Eur. J. Endocrinol. 156, 117-122. doi: 10.1530/eje.1.02318

Zeitoun, K., Takayama, K., Sasano, H., Suzuki, T., Moghrabi, N., Andersson, S., et al. (1998). Deficient $17 \beta$-hydroxysteroid dehydrogenase type 2 expression in endometriosis: failure to metabolize $17 \beta$-estradiol. J. Clin. Endocrinol. Metab. $83,4474-4480$.

Zeng, C., Matsuda, K., Jia, W. H., Chang, J., Kweon, S. S., Xiang, Y. B., et al. (2016). Identification of susceptibility loci and genes for colorectal cancer risk. Gastroenterology 150, 1633-1645. doi: 10.1053/j.gastro.2016.02.076

Zhang, J., Yin, Y., Niu, X. M., Liu, Y., Garfield, D., Chen, S. F., et al. (2013). $\mathrm{CYP}_{19} \mathrm{~A}_{1}$ gene polymorphisms and risk of lung cancer. J. Int. Med. Res. 41, 735-742. doi: 10.1177/0300060513477291

Zhao, H., Zhou, L., Shangguan, A. J., and Bulun, S. E. (2016). Aromatase expression and regulation in breast and endometrial cancer. J. Mol. Endocrinol. 57 R19-33. doi: 10.1530/JME-15-0310

Zhongyi, S., Rantakari, P., Lamminen, T., Toppari, J., and Poutanen, M. (2007). Transgenic male mice expressing human hydroxysteroid dehydrogenase 2 indicate a role for the enzyme independent of its action on sex steroids. Endocrinology 148, 3827-3836. doi: 10.1210/en.2007-0365

Zwart, W., Theodorou, V., Kok, M., Canisius, S., Linn, S., and Carroll, J. S. (2011). Oestrogen receptor-co-factor-chromatin specificity in the transcriptional regulation of breast cancer. EMBO J. 30, 4764-4776. doi: $10.1038 /$ emboj. 2011.368

Conflict of Interest Statement: PK and TL are employees of Forendo Pharma Ltd.

The remaining authors declare that the research was conducted in the absence of any commercial or financial relationships that could be construed as a potential conflict of interest.

Copyright (c) 2018 Konings, Brentjens, Delvoux, Linnanen, Cornel, Koskimies, Bongers, Kruitwagen, Xanthoulea and Romano. This is an open-access article distributed under the terms of the Creative Commons Attribution License (CC BY). The use, distribution or reproduction in other forums is permitted, provided the original author(s) and the copyright owner(s) are credited and that the original publication in this journal is cited, in accordance with accepted academic practice. No use, distribution or reproduction is permitted which does not comply with these terms. 